\title{
Axially Grooved and Arterial Heat Pipe Testing and Numerical Analysis
}

\author{
by \\ Michel Garcia \\ B.Eng., Carleton University

\begin{abstract}
A Thesis submitted to
the Faculty of Graduate Studies and Research

in partial fulfilment of

the requirements for the degree of
\end{abstract} \\ Master of Applied Science \\ Ottawa-Carleton Institute for \\ Mechanical and Aerospace Engineering
Department of Mechanical and Aerospace Engineering
Carleton University \\ Ottawa, Ontario, Canada
}

April 2006

Copyright (C)

2006 - Michel Garcia 


$\begin{array}{ll}\begin{array}{l}\text { Library and } \\ \text { Archives Canada }\end{array} & \begin{array}{l}\text { Bibliothèque et } \\ \text { Archives Canada }\end{array} \\ \begin{array}{l}\text { Published Heritage } \\ \text { Branch }\end{array} & \begin{array}{l}\text { Direction du } \\ \text { Patrimoine de l'édition }\end{array} \\ \begin{array}{l}\text { 395 Wellington Street } \\ \text { Ottawa ON K1A ON4 }\end{array} & \begin{array}{l}\text { 395, rue Wellington } \\ \text { Ottawa ON K1A ON4 } \\ \text { Canada }\end{array}\end{array}$

Your file Votre référence ISBN: 978-0-494-16458-7 Our file Notre référence ISBN: 978-0-494-16458-7

NOTICE:

The author has granted a nonexclusive license allowing Library and Archives Canada to reproduce, publish, archive, preserve, conserve, communicate to the public by telecommunication or on the Internet, loan, distribute and sell theses worldwide, for commercial or noncommercial purposes, in microform, paper, electronic and/or any other formats.

The author retains copyright ownership and moral rights in this thesis. Neither the thesis nor substantial extracts from it may be printed or otherwise reproduced without the author's permission.
AVIS:

L'auteur a accordé une licence non exclusive permettant à la Bibliothèque et Archives Canada de reproduire, publier, archiver, sauvegarder, conserver, transmettre au public par télécommunication ou par l'Internet, prêter, distribuer et vendre des thèses partout dans le monde, à des fins commerciales ou autres, sur support microforme, papier, électronique et/ou autres formats.

L'auteur conserve la propriété du droit d'auteur et des droits moraux qui protège cette thèse. $\mathrm{Ni}$ la thèse ni des extraits substantiels de celle-ci ne doivent être imprimés ou autrement reproduits sans son autorisation.
In compliance with the Canadian

Privacy Act some supporting forms may have been removed from this thesis.

While these forms may be included in the document page count, their removal does not represent any loss of content from the thesis.
Conformément à la loi canadienne sur la protection de la vie privée, quelques formulaires secondaires ont été enlevés de cette thèse.

Bien que ces formulaires aient inclus dans la pagination, il n'y aura aucun contenu manquant.

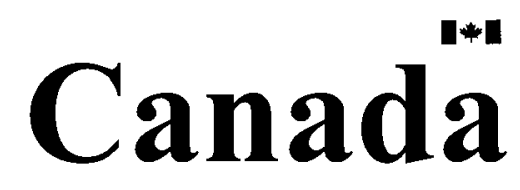




\section{Abstract}

Testing of an axially grooved and an arterial heat pipe was performed under ambient conditions. Steady-state axial temperature distribution as a function of heat input and sink temperature was determined. Variable inclination testing with $5 \mathrm{~W}$ input power was performed to determine the dryout angle. Horizontal testing with variable power input was performed to determine the capillary limit. The effective thermal conductivity of each heat pipe as a function of heat input and sink temperature was determined.

Numerical modelling of the axially grooved heat pipe tested was performed. Poiseuille number as a function of contact angle and attachment point was determined. A capillary limit model was developed based on the groove geometry and external heat pipe dimensions. The parametric study of attachment point and contact angle variation led to the prediction of maximum heat transfer. Satisfactory agreement was found between the experiment and numerical model. 


\section{Acknowledgments}

I would like to thank my supervisor, Professor Tarik Kaya for being there to answer my many questions. Funding provided by CRESTech, MMO, and NSERC was appreciated. I am grateful to the Carleton Mechanical and Aerospace Engineering machine shop and administrative staff who gave me much of their time. I am also thankful to my family and friends for their support. 


\section{Table of Contents}

Abstract $\quad$ iii

Acknowledgments $\quad$ iv

Table of Contents $\quad$ v

List of Tables viii

List of Figures $\quad$ ix

1 Introduction 1

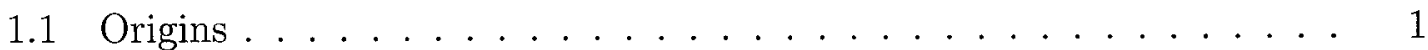

1.2 Thermodynamic Cycle ................... 2

1.3 Effective Thermal Conductivity . . . . . . . . . . . 3

1.4 Testing ........................... 4

1.4.1 Gravitational Tilt Testing ............. 5

1.5 Importance of the Poiseuille Number . . . . . . . . . . . . 6

1.6 Liquid-Vapour Counterflow . . . . . . . . . . . . . . 7

1.7 Modelling of Re-Entrant Grooves . . . . . . . . . . . . 8

1.8 Heat Transfer Limits . . . . . . . . . . . . . . . . . . . 9 9

1.8.1 Capillary Limit . . . . . . . . . . . . . 10 
1.9 Arterial Heat Pipes . . . . . . . . . . . . . . . . 11

2 Experimental Investigation 13

2.1 Test Setup Summary . . . . . . . . . . . . . . . . . . . . 13

2.2 Experimental Equipment . . . . . . . . . . . . . . . 14

2.2 .1 General Setup . . . . . . . . . . . . . . . . . . . . 14

2.2 .2 Heat Pipes . . . . . . . . . . . . . . . . . 15

2.2 .3 Heating . . . . . . . . . . . . . . . . . . . . . 19

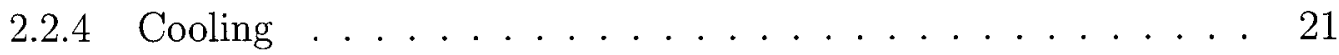

2.2 .5 Insulation and Support . . . . . . . . . . . . . . . . 22

2.2 .6 Tilting Mechanism . . . . . . . . . . . . . . . . . . . . 23

2.2.7 Data Acquisition . . . . . . . . . . . . . 26

2.3 Experimental Process . . . . . . . . . . . . . . . 30

2.3.1 Steady-State Response . . . . . . . . . . . . . . . . . 30

2.3.2 Transient Response . . . . . . . . . . . . . . . . . . . 30

2.3 .3 Testing Performed $\ldots \ldots \ldots \ldots \ldots$

2.4 Experimental Results . . . . . . . . . . . . . . . . . 32

2.4.1 Temporal Temperature Variation . . . . . . . . . . . . 32

2.4.2 Spatial Temperature Variation . . . . . . . . . . . . . . 42

2.4.3 Effective Thermal Conductivity . . . . . . . . . . 51

2.4 .4 Heat Balance . . . . . . . . . . . . . . . . . . . . 53

2.4.5 Inclination Testing . . . . . . . . . . . . 57

2.4 .6 Horizontal Dryout $\ldots \ldots \ldots \ldots \ldots$

2.4 .7 AHP Heater Location Variation . . . . . . . . . . . . 59

3 Numerical Modelling $\quad 68$

3.1 Axially Grooved Heat Pipe Capillary Limit Theory . . . . . . . . 68 vi 
3.2 Liquid Groove . . . . . . . . . . . . . . . . . . . . . . 69

3.2 .1 Global Pressure Losses . . . . . . . . . . . . . . . . . 69

3.2 .2 Groove Modelling . . . . . . . . . . . . . . . . . . . 72

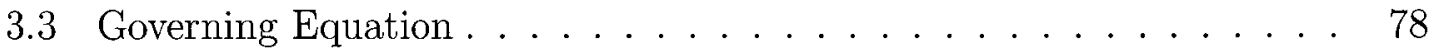

3.3 .1 Boundary Conditions . . . . . . . . . . . . . . . . . 82

3.4 Solving the Governing Equation . . . . . . . . . . . . 85

3.4.1 Finite Difference Model . . . . . . . . . . . . . . . . . . . 85

3.4 .2 Finite Element Model _. . . . . . . . . . . . . . . . . 90

3.5 Vapour Flow . . . . . . . . . . . . . . . . . . . 92

3.6 Combining Liquid and Vapour Flows _ . . . . . . . . . . . 93

3.7 Capillary Limit Code Structure $\ldots \ldots \ldots \ldots$

3.8 Numerical Results. . . . . . . . . . . . . . . . . . . . . 97

3.8.1 Velocity Fields Resulting from Different Boundary Conditions 98

3.8 .2 Contact Angle Variation . . . . . . . . . . . . 100

3.8.3 Attachment Point Variation . . . . . . . . . . . . . 105

3.8.4 Comparison with Experiment . . . . . . . . . . . . 109

4 Conclusions and Recommendations 112

4.1 Conclusions . . . . . . . . . . . . . . . . . . . . . . . 112

4.1 .1 Experimental Study $\ldots \ldots \ldots 112$

4.1 .2 Numerical Study . . . . . . . . . . . . . . . . . . . 113

4.2 Recommendations . . . . . . . . . . . . . . . . . . . . . 114

4.2 .1 Experimental Recommendations . . . . . . . . . . . . . . 114

4.2 .2 Numerical Recommendations . . . . . . . . . . . . 116

$\begin{array}{ll}\text { References } & 118\end{array}$

vii 


\section{List of Tables}

2.1 Specific Heat Coefficients, Ref.[22] . . . . . . . . . . 55

2.2 Property Uncertainty . . . . . . . . . . . . . . 56

3.1 Four Interfacial Cases . . . . . . . . . . . . . . . . 79 


\section{List of Figures}

1.1 Early Heat Pipe Depiction, Ref.[1] . . . . . . . . . . . . 1

1.2 Heat Pipe Cycle, Ref.[2] . . . . . . . . . . . . . 3

1.3 Typical Experimental Setup, Ref.[4] . . . . . . . . . . . 4

1.4 Effect of Puddling on Actual Elevation, Ref.[5] . . . . . . . . . . 5

1.5 Liquid-Vapour Counterflow, Ref.[10] . . . . . . . . . . . 8

1.6 Obtaining Shear and Average Velocity Semi-Analytically, Ref.[11] . . 9

1.7 Re-Entrant Axially Grooved Heat Pipe Cross-Section, Ref.[9] . . . . . 10

1.8 FEM Grid for a Re-Entrant Groove, Ref.[13] . . . . . . . . . . . . . 11

1.9 Heat Transfer Limits Encountered with Temperature, Ref.[14] . . . 12

1.10 Typical Arterial Heat Pipe Cross-Section, Ref.[17] . . . . . . . . . 12

2.1 AGHP Setup without Insulation . . . . . . . . . . . . . 14

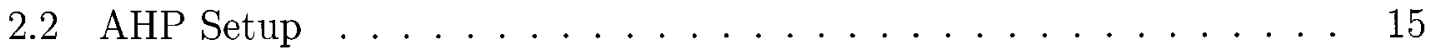

2.3 Test Setup Schematic . . . . . . . . . . . . . . . 16

2.4 AGHP Drawing (all dimensions in $\mathrm{mm}$ ) . . . . . . . . . . 17

2.5 AHP Drawing (all dimensions in $\mathrm{mm}$ ) . . . . . . . . . 18

2.6 Low Power Film Heater . . . . . . . . . . . . . . . . . . 20

2.7 Cartridge Heater Block Cross-Section . . . . . . . . . . . . . 21

2.8 Omega FL-2068 Flowmeter . . . . . . . . . . . . . . . . . . . . . . . . 21

2.9 Tilting Mechanism .................. 24 
2.10 Heat Pipe Stand Adjustable Lift Mechanism . . . . . . . . . . . . . 28

2.11 Clinometer used to Measure Tilt Angle . . . . . . . . . . . . . 29

2.12 Thermocouple Locations . . . . . . . . . . . . . . . 29

2.13 Variation of Heat Input and Sink Temperature . . . . . . . . . . . . 32

2.14 AGHP, Average Temperature, Sink $5^{\circ} \mathrm{C} \ldots \ldots . \ldots . . \ldots 34$

2.15 AGHP, Average Temperature, Sink $10^{\circ} \mathrm{C} \ldots \ldots . . . . . . . .35$

2.16 AGHP, Average Temperature, Sink $15^{\circ} \mathrm{C} \ldots \ldots . \ldots 35$

2.17 AGHP, Average Temperature, Source $0 \mathrm{~W} \ldots \ldots$. . . . . . . 36

2.18 AGHP, Average Temperature, Source $40 \mathrm{~W}$. . . . . . . . . 36

2.19 AGHP, Average Temperature, Source $80 \mathrm{~W}$. . . . . . . . . . . 37

2.20 AGHP, Average Temperature, Source 120 W . . . . . . . . . . . 37

2.21 AGHP, Average Temperature, Source $160 \mathrm{~W}$. . . . . . . . . 38

2.22 AHP, Average Temperature, Sink $5{ }^{\circ} \mathrm{C} \ldots \ldots . . . \ldots 38$

2.23 AHP, Average Temperature, Sink $10^{\circ} \mathrm{C} \ldots \ldots . \ldots . . . . . .39$

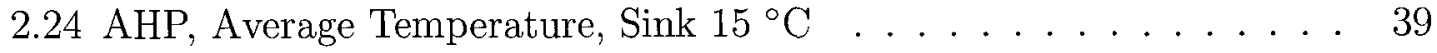

2.25 AHP, Average Temperature, Source $0 \mathrm{~W} \ldots . . . . . . . .40$

2.26 AHP, Average Temperature, Source $80 \mathrm{~W}$. . . . . . . . . . 40

2.27 AHP, Average Temperature, Source 120 W . . . . . . . . . . . . 41

2.28 AHP, Average Temperature, Source 160 W . . . . . . . . . . . 41

2.29 AGHP, Station Temperature, Sink $5^{\circ} \mathrm{C} \ldots \ldots . . . . . . . .43$

2.30 AGHP, Station Temperature, Sink $10^{\circ} \mathrm{C} \ldots \ldots . . . . . . .44$

2.31 AGHP, Station Temperature, Sink $15^{\circ} \mathrm{C} \ldots \ldots . . . . . . . .44$

2.32 AGHP, Station Temperature, Source 0 W . . . . . . . . . 45

2.33 AGHP, Station Temperature, Source $40 \mathrm{~W} \ldots \ldots . . . . . .45$

2.34 AGHP, Station Temperature, Source $80 \mathrm{~W} \ldots \ldots$. . . . . . . . 46

2.35 AGHP, Station Temperature, Source $120 \mathrm{~W} \ldots \ldots$. . . . . . . 46 
2.36 AGHP, Station Temperature, Source 160 W . . . . . . . . . . . 47

2.37 AHP, Station Temperature, Sink $5{ }^{\circ} \mathrm{C} \ldots \ldots . \ldots 7$

2.38 AHP, Station Temperature, Sink $10^{\circ} \mathrm{C} \ldots \ldots . . . . . . . .48$

2.39 AHP, Station Temperature, Sink $15{ }^{\circ} \mathrm{C} \ldots \ldots . . \ldots 48$

2.40 AHP, Station Temperature, Source 0 W . . . . . . . . . . 49

2.41 AHP, Station Temperature, Source 80 W . . . . . . . . . . . 49

2.42 AHP, Station Temperature, Source $120 \mathrm{~W} \ldots \ldots . . . . . . .50$

2.43 AHP, Station Temperature, Source 160 W . . . . . . . . . . . 50

2.44 AGHP Effective Thermal Conductivity . . . . . . . . . . . . 52

2.45 AHP Effective Thermal Conductivity . . . . . . . . . . . . 53

2.46 Heat Input Measurement Uncertainty . . . . . . . . . . . . . 57

2.47 AGHP, Average Temperature, Source 5 W, Tilt Dryout . . . . . . . 58

2.48 AHP, Average Temperature, Source 5 W, Tilt Dryout . . . . . . . . 59

2.49 AGHP, Average Temperature, $\psi=-90^{\circ}$ tilt . . . . . . . . 60

2.50 AGHP, Average Temperature, $\psi=0^{\circ}$ tilt . . . . . . . . . . . 61

2.51 AGHP, Station Temperature, $\psi=-90^{\circ}$ tilt $\ldots \ldots . \ldots 61$

2.52 AGHP, Station Temperature, $\psi=0^{\circ}$ tilt . . . . . . . . . 62

2.53 AHP, Average Temperature, $\psi=-90^{\circ}$ tilt . . . . . . . . . 62

2.54 AHP, Average Temperature, $\psi=0^{\circ}$ tilt . . . . . . . . . 63

2.55 AHP, Station Temperature, $\psi=-90^{\circ}$ tilt $\ldots \ldots . \ldots 3$

2.56 AHP, Station Temperature, $\psi=0^{\circ}$ tilt . . . . . . . . . 64

2.57 AGHP, Average Temperature, Horizontal Dryout . . . . . . . . 64

2.58 AHP, Average Temperature, Horizontal Dryout . . . . . . . . . 65

2.59 AGHP, Manufacturer Data Comparison . . . . . . . . . . . 65

2.60 AHP, Manufacturer Data Comparison . . . . . . . . . . 66

2.61 Possible AHP Heater Configurations . . . . . . . . . . . . 66 
2.62 AHP, Average Temperature, Heater below Evaporator . . . . . . . . . 67

3.1 Axial Groove Meniscus . . . . . . . . . . . . . . . . . . . 69

3.2 Gravitational Influence on Line Segments . . . . . . . . . . . . 70

3.3 Mitutoyo Profile Projector . . . . . . . . . . . . . 73

3.4 AGHP Cross-Section Used for Measurement . . . . . . . . . . . 74

3.5 Curve Fit to Measured Points . . . . . . . . . . . . . . . . 75

3.6 Outlined Area Deficit . . . . . . . . . . . . . . 76

3.7 Four Interfacial Cases . . . . . . . . . . . . . . . . . . . 77

3.8 Angle Layout for Case $2 \ldots \ldots$. . . . . . . . . . . . . . 78

3.9 Balance Between Pressure and Shear . . . . . . . . . . . . . . 80

3.10 Area to Perimeter Variation . . . . . . . . . . . . . . . 82

3.11 Interface Length Variation . . . . . . . . . . . . . 83

3.12 Liquid Groove Boundary Segments . . . . . . . . . . . . . 84

3.13 Liquid-Vapour Interface Coordinates . . . . . . . . . . . . 87

3.14 Mandatory Discretization Scheme . . . . . . . . . . . . 88

3.15 Scheme Similarity Based on Domain Origin . . . . . . . . . . . 89

3.16 Finite Element Mesh, $x^{*}=0.7, \theta=45^{\circ} \ldots \ldots \ldots$

3.17 Determining Interfacial Shear . . . . . . . . . . . . . . . . . 91

3.18 Axial Vapour Control Volume . . . . . . . . . . . . 93

3.19 Assumed Axial Heat Transfer . . . . . . . . . . . . . . . 95

3.20 Capillary Limit Algorithm Flowchart . . . . . . . . . . . . . . . 96

3.21 No Slip Velocity Contour, $x^{*}=0.64, \theta=0^{\circ} \ldots \ldots$. . . . . . 98

3.22 No Shear Velocity Contour, $x^{*}=0.74, \theta=0^{\circ} \ldots \ldots$. . . . . . 98

3.23 Vapour Counterflow Shear Velocity Contour, $x^{*}=0.84, \theta=0^{\circ}$. . . 99

3.24 Contact Angle Variation at $x^{*}=0.64,0.74$, and $0.84 \ldots \ldots$

3.25 Po Variation with Contact Angle, No Slip . . . . . . . . . . . . . . 101

xii 
3.26 Capillary Limit Variation with Contact Angle, No Slip . . . . . . 102

3.27 Po Variation with Contact Angle, Vapour Counterflow . . . . . . . 103

3.28 Capillary Limit Variation with Contact Angel, Vapour Counterflow . 104

3.29 Po Variation with Attachment Point, No Slip . . . . . . . . . 105

3.30 Capillary Limit Variation with Attachment Point, No Slip . . . . 106

3.31 Po Variation with Attachment Point, Vapour Counterflow . . . . . 107

3.32 Capillary Limit Variation with Attachment Point, Vapour Counterflow 108

3.33 Capillary Limit Variation with Inclination Angle, No Slip . . . . . . 109

3.34 Capillary Limit Variation with Inclination Angle, Vapour Counterflow 110 


\section{Nomenclature}

\section{Roman}

$A \quad$ Heat pipe cross-sectional area $\mathrm{m}^{2}$

$A_{d} \quad$ Area deficit $\quad \mathrm{m}^{2}$

$A_{l} \quad$ Liquid groove cross-sectional area $\quad \mathrm{m}^{2}$

$c_{p} \quad$ Specific heat at constant pressure $\quad \frac{\mathrm{kJ}}{\mathrm{kgK}}$

$\frac{d P_{l}}{d z} \quad$ Axial liquid pressure gradient $\quad \frac{\mathrm{N}}{\mathrm{m}^{3}}$

$f_{l} \quad$ Liquid frictional drag coefficient

$\vec{g} \quad$ Gravitational acceleration $\quad \frac{m}{s^{2}}$

$h_{f g} \quad$ Latent heat of vapourization $\quad \frac{\mathrm{J}}{\mathrm{kg}}$

I Current $\quad$ A

$L \quad$ Heat pipe length $\quad$ m

$L_{\text {eff }} \quad$ Effective length $\quad \mathrm{m}$ 
$L_{g} \quad$ Length of liquid groove $\quad \mathrm{m}$

$L_{\text {int }} \quad$ Liquid-vapour interface length $\quad \mathrm{m}$

$\hat{n} \quad$ Direction normal to liquid-vapour interface

$n \quad$ Number of grooves

$P \quad$ Liquid groove cross-sectional perimeter $\quad \mathrm{m}$

$\begin{array}{lll}P_{c} \quad \text { Capillary pressure } & \mathrm{Pa}\end{array}$

$\begin{array}{lll}P_{l} \quad \text { Liquid pressure } & \mathrm{Pa}\end{array}$

$P_{v} \quad$ Vapour pressure $\quad P a$

$P_{l} \quad$ Liquid groove Poiseuille number

Q Axial heat transfer $\quad$ W

$Q_{\text {in }}$ Heat input to evaporator $\quad$ W

Qout Condenser heat output $\quad$ W

$R_{1}, R_{2}$ Perpendicular interfacial radii of curvature $\quad \mathrm{m}$

$R_{v} \quad$ Vapour groove radius $\quad \mathrm{m}$

$r_{h l} \quad$ Hydraulic radius $\quad \mathrm{m}$ 
$r_{h p} \quad$ Heat pipe radius of curvature

$R e_{l} \quad$ Axial groove liquid Reynolds number

T Temperature

${ }^{\circ} \mathrm{C}$ or $\mathrm{K}$

$T_{\text {in }} \quad$ Coolant temperature entering heat exchanger

${ }^{\circ} \mathrm{C}$ or $\mathrm{K}$

$T_{\text {out }}$ Coolant temperature exiting heat exchanger

${ }^{\circ} \mathrm{C}$ or $\mathrm{K}$

$u \quad$ Axial groove liquid velocity

$\frac{\mathrm{m}}{\mathrm{s}}$

$\dot{V} \quad$ Volumetric flow rate

$\frac{\mathrm{m}^{3}}{\mathrm{~s}}$

V Voltage

$v \quad$ specific volume

$\frac{\mathrm{m}^{3}}{\mathrm{~kg}}$

$w \quad$ Groove width

$\mathrm{m}$

$\Delta x \quad$ Independent variable numerical grid increment

$\mathrm{m}$

$x \quad$ Liquid groove coordinate along axis of symmetry

$\mathrm{m}$

$\Delta y \quad$ Dependent variable numerical grid increment

$\mathrm{m}$

$y \quad$ Liquid groove coordinate normal to axis of symmetry

$\mathrm{m}$

$z^{\prime} \quad$ Coordinate relating straight and curved heat pipe sections

$\mathrm{m}$

xvi 


\section{Greek}

$\delta \quad$ Variable uncertainty

$\gamma \quad$ Heat pipe curvature angle

$\operatorname{rad}$

$\lambda \quad$ Groove slope tangent angle at attachment point

$\operatorname{rad}$ or ${ }^{\circ}$

$\lambda_{\text {eff }} \quad$ Effective thermal conductivity

$\frac{\mathrm{W}}{\mathrm{mK}}$

$\mu \quad$ Dynamic viscosity

$\frac{\mathrm{kg}}{\mathrm{ms}}$

$\nabla \quad$ Gradient operator

$\frac{1}{\mathrm{~m}}$

$\rho_{c} \quad$ Coolant density

$\frac{\mathrm{kg}}{\mathrm{m}^{3}}$

$\sigma \quad$ Surface tension

$\frac{\mathrm{N}}{\mathrm{m}}$

$\sigma_{R} \quad$ Angle made by $x^{*}$ axis and $R_{1}$ at $x^{*}$

$\tau \quad$ Shear stress

$\mathrm{Pa}$

$\theta \quad$ Contact wetting angle

$\operatorname{rad}$ or $^{\circ}$

$\Upsilon \quad$ Heater power phase angle

$\circ$

$\xi \quad$ Angle between $\hat{n}$ and $x^{*}$ axis

$\circ$

$\zeta \quad \frac{\Delta x}{\Delta y}$

xvii 
$\psi \quad$ Adverse heat pipe inclination angle

\section{Subscripts}

ad Adiabatic

co Condenser

eg Ethylene glycol

ev Evaporator

lv Liquid-vapour interface

sl Solid-liquid interface

V Vapour

w Water

\section{Superscripts}

$\mathrm{k} \quad$ Iteration parameter

* Non-dimensionalization indicator

\section{Acronyms}

AGHP Axially grooved heat pipe

xviii 
AHP Arterial heat pipe

FEM Finite element method

GPM Gallon per minute

GUI Graphical user interface

LPM Litre per minute

PDE Partial differential equation

RMS Root mean square 


\section{Chapter 1}

\section{Introduction}

\section{$1.1 \quad$ Origins}

The heat pipe has been around since 1963, Ref.[1] when Los Alamos National Laboratories reported the construction and operation of "Structures of Very High Thermal Conductance," initially depicted in Figure 1.1.

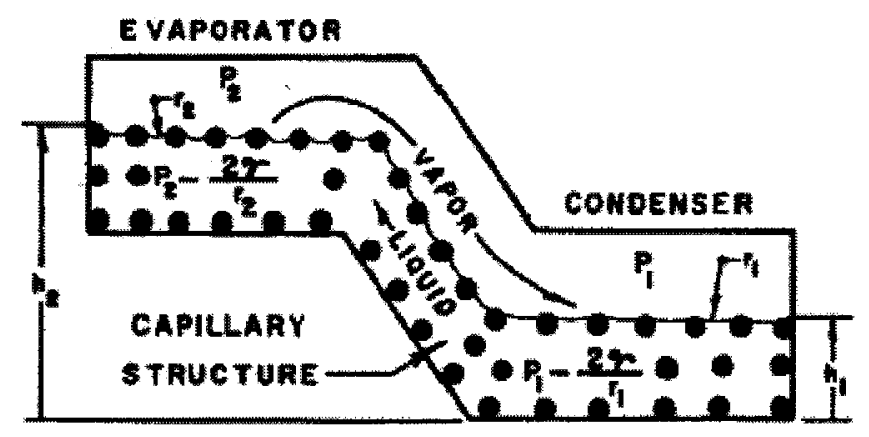

Figure 1.1: Early Heat Pipe Depiction, Ref.[1]

Paths of high thermal conductivity are required in many engineering systems. Metals such as silver and copper transfer heat well by conduction, but their low specific thermal conductivity make them costly to include in spacecraft as pure conductors. 
Heat pipes transfer heat passively and more effectively than purely conductive metallic structures having the same dimensions.

The internal structure of a heat pipe is responsible for keeping the liquid and vapour phases of the working fluid separate inside the adiabatic section and allowing for a phase change to occur in the evaporator and condenser. This is primarily done by manipulating the effects of surface tension with the applied source and sink, and wick or groove geometry.

\subsection{Thermodynamic Cycle}

The heat pipe cycle can be more thoroughly understood in the context of thermodynamics. A two-phase temperature-entropy diagram seen in Figure 1.2 can be used to break the basic cycle down into more easily comprehensible sections. The working fluid in liquid form enters the evaporator at $1 \mathrm{~A}$ and is raised to the highest operating temperature within the cycle at 2B. Evaporation and entrance into the vapour groove occur between $2 \mathrm{~B}$ and $2 \mathrm{C}$. Vapour travels from $2 \mathrm{C}$ to $3 \mathrm{D}$ along the adiabatic section. Condensation and entrance into the liquid groove(s) occur between 3D and 3E. Finally, the liquid travels along the adiabatic section from $3 \mathrm{E}$ to $1 \mathrm{~A}$ closing the cycle loop.

Heat pipes transfer energy nearly isothermally because they use their working fluid's latent heat of vapourization. Heat input vapourizes the working fluid and increases its pressure, causing it to seek a region of lower pressure. Two options exist for the vapour assuming a rigid wall. The vapour could either bypass or enter the liquid region. The latter does not occur because surface tension acts as a mechanical diode allowing liquid to enter the vapour region, but prohibiting vapour from entering 


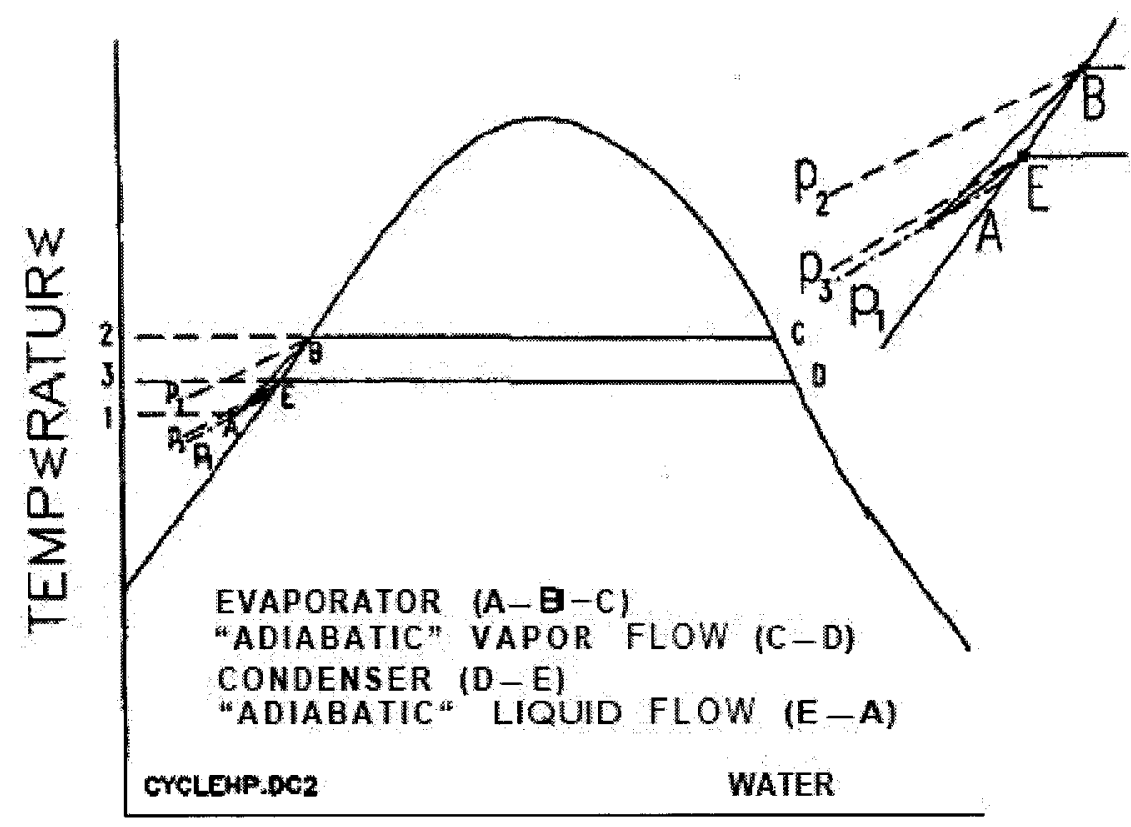

ENTROPY

Figure 1.2: Heat Pipe Cycle, Ref.[2]

the liquid region. Once the working fluid reaches the condenser, heat is output, resulting in a phase change. The interfacial radius of curvature increases due to the increase of fluid in the liquid state. This reduces the surface tension and allows the vapour to cross the interface and condense.

\subsection{Effective Thermal Conductivity}

In order to compare how efficiently various devices transfer heat, the effective thermal conductivity is evaluated. A functioning heat pipe can have an effective thermal conductivity orders of magnitude greater than a copper rod of equivalent dimensions. Modelling to predict the effective thermal conductivity and comparing with experimental data may require extensive work because of specific thermocouple placement. 
A resistance network model approach introduced in Ref.[3] can be used to take into account the heater, heat exchanger, and pipe casing. Such models typically assume simple shapes for the components such as annular heaters and heat exchangers. Given that asymmetric saddle heaters and coolers are used in this work's experimental setup, a worthwhile resistance model would most likely require the inclusion of a numerical model to handle the more involved geometries.

\section{$1.4 \quad$ Testing}

It is important to know how a heat pipe will behave prior to intended use. Laboratory testing gives insight to characterize heat pipe performance. A typical heat pipe test setup is seen in Figure 1.3 and can be expanded to test with various other inputs

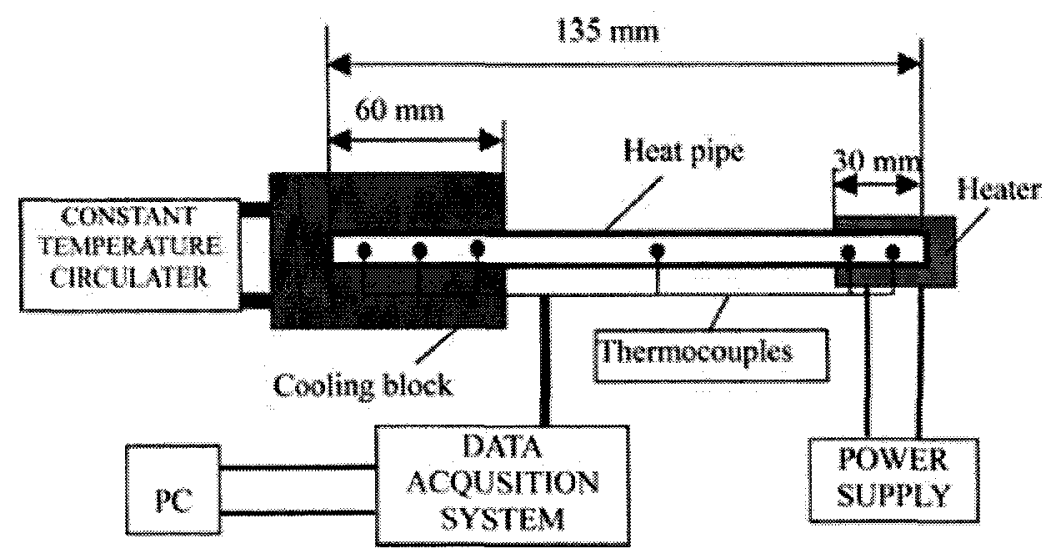

Figure 1.3: Typical Experimental Setup, Ref.[4]

such as inclination, vacuum, or vibrations. 


\subsubsection{Gravitational Tilt Testing}

How a heat pipe reacts under a body force is important when characterizing its performance for terrestrial applications. Space based applications can also benefit from knowledge related to body forces since spacecraft accelerations result in a body force.

Testing under an adverse gravitational field with an overcharged heat pipe increases susceptibility to puddling, Ref.[5]; i.e., the formation of a liquid reservoir at the condenser which decreases the height the liquid has to travel in order to maintain the cycle. The variation of the effective elevation with actual elevation due to puddling can be seen in Figure 1.4 for different filling volumes. Puddling would not

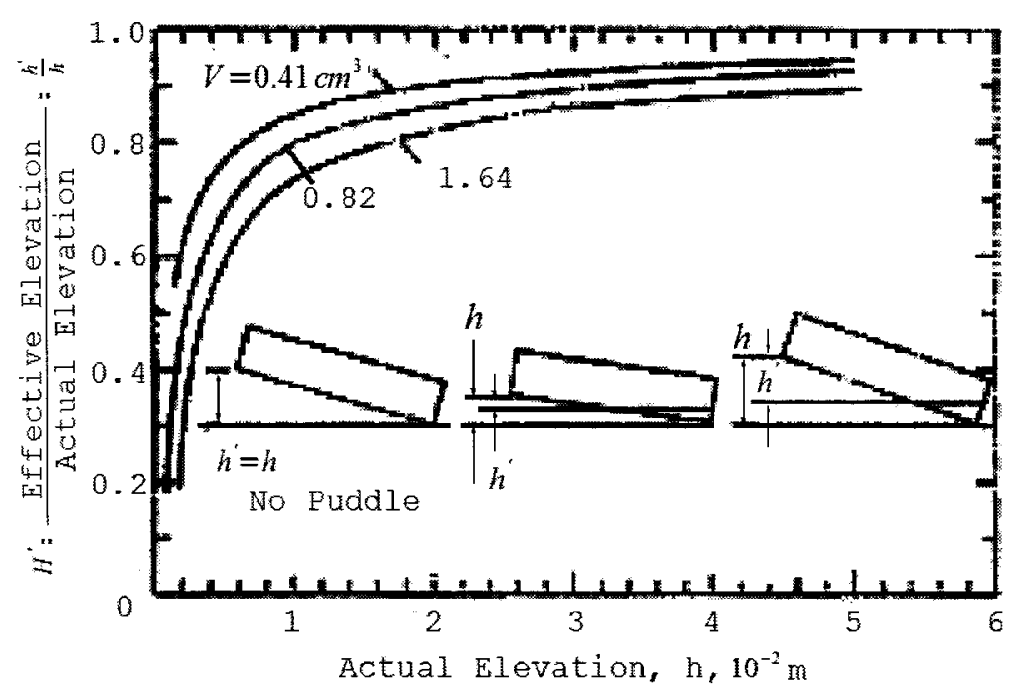

Figure 1.4: Effect of Puddling on Actual Elevation, Ref.[5]

occur in a 0 -g environment, thereby assuming terrestrial results would lead to an overestimation of service performance if no correction is implemented.

The manner in which the heat pipes tested in this work were tilted would not produce the puddling effect mentioned in Ref.[5] because the condenser does not 
point downwards during tilt testing due to the u-shaped nature of both pipes. The effects of puddling could be investigated by tilting the pipe about another axis.

\subsection{Importance of the Poiseuille Number}

The groove structure is significant in determining liquid flow behaviour inside a heat pipe. A complicated groove shape must often be chosen in order to achieve both a high capillary pressure and a high mass flow rate. This rules out simple geometries where analytical solutions are obtainable.

Assuming steady-state, fully developed, incompressible flow allows for use of the Poiseuille number to characterize the flow for a given geometry. The magnitude of the pressure gradient is determined by the applied heat using the first law of thermodynamics given a phase change, but is independent of the Poiseuille number.

The work of Poiseuille Ref.[6] in the 18th century was invaluable to the understanding of conduit flow. The Poiseuille number, which is solely dependent on crosssectional geometry is derived assuming a balance between the applied pressure and wall shear stress, Ref.[7].

The examination of complex, yet not arbitrary geometries was made in Ref.[8]. Analytical solutions to the Poisson equation were provided. A characteristic length different from the hydraulic diameter was provided to create dimensionless groups that were weak functions of shape.

Examination of Poiseuille number variation with heat pipe groove geometry was performed in Ref.[9], where the variation in the contact angle was considered less 
important compared to the attachment point when evaluating the flow variables. It will later be shown that heat transfer is significantly dependent on contact angle. Heat transfer is determined using both the flow variables and interfacial geometry. The inclusion of the contact angle in a parametric analysis of the capillary limit is crucial if no model is used for its prediction.

\subsection{Liquid-Vapour Counterflow}

Pressure change in the liquid flow can be modelled as being the combinational result of frictional losses due to the solid-liquid interface and the shearing action of the vapour counterflow.

Vapour induced shear at the liquid-vapour interface can be introduced in order to more accurately depict the physics of heat pipe flow, Ref.[10]. Figure 1.5 shows the resultant flow when ignoring and accounting for the liquid-vapour interactive counterflow. The liquid and vapour flows can be solved separately and joined at the interface with a common shear stress. Knowledge of the interfacial behaviour is critical in determining the retarding effect on the liquid flow.

The work in Ref.[11] finds the variation in Poiseuille number for trapezoidal grooves given an applied shear stress at the liquid-vapour interface. Given the linear relationship between wall shear and average velocity in Poiseuille duct flow and in combined Poiseuille-Couette flow between plates Ref.[7], this was assumed true for groove flow with shear at the liquid-vapour interface. A semi-analytic solution is illustrated in Figure 1.6 where the line defining this relationship is obtained. A similar analysis for sinusoidal grooves was performed in Ref.[12]. 


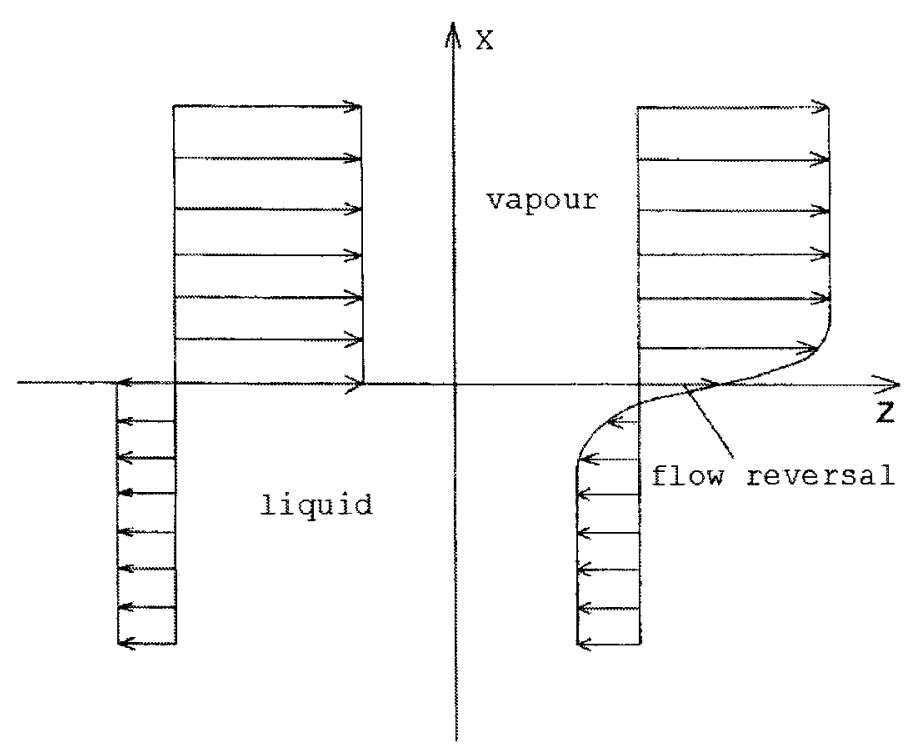

Figure 1.5: Liquid-Vapour Counterflow, Ref.[10]

\subsection{Modelling of Re-Entrant Grooves}

A high heat transfer rate is achieved in a heat pipe by providing a structure leading to a high maximum capillary pressure and low axial pressure loss. Maximizing capillary pressure in an axially grooved heat pipe is done by minimizing the width of the liquid-vapour interface. This alone leads to a large axial pressure drop. Combining a circular shape which inherently has a low axial pressure drop with a slot which has high maximum capillary pressure reduces the axial pressure drop. This type of geometry is known as a re-entrant groove and has been used in space with success. Re-entrant grooves can be seen in Figure 1.7 as part of a circumferentially axially grooved heat pipe. The large base of the re-entrant groove also allows for a higher mass flow rate to carry more heat.

Re-entrant groove analysis in Ref.[13] combines a two-dimensional radial heat transfer model with a one-dimensional liquid flow model. The liquid flow model 


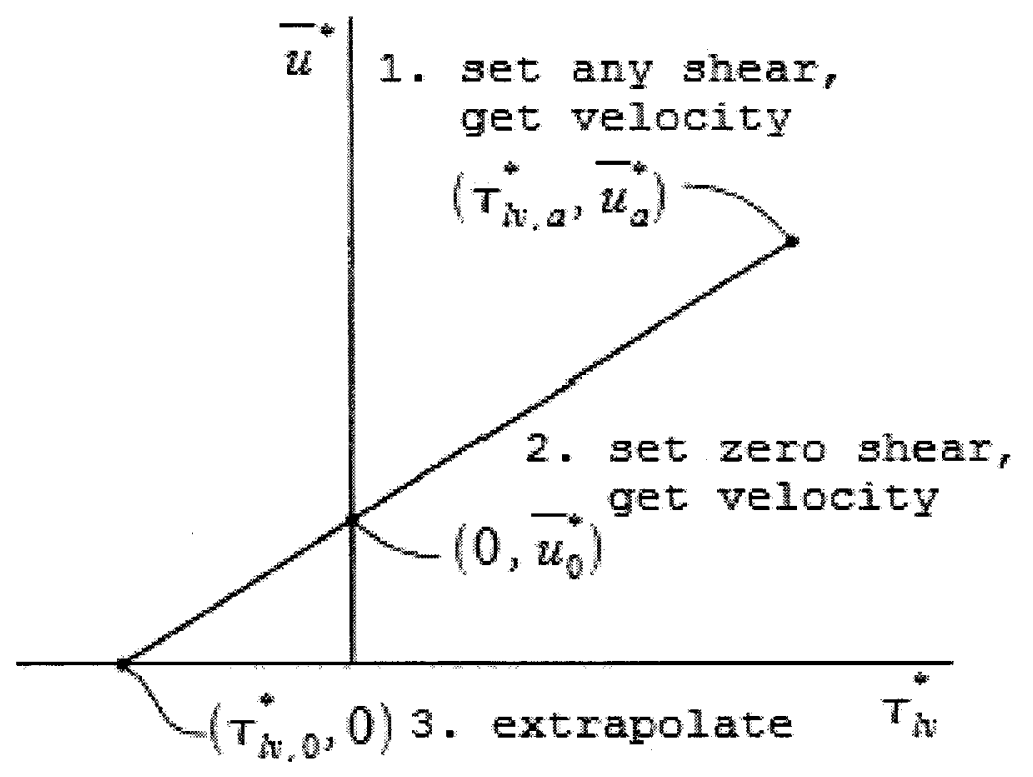

Figure 1.6: Obtaining Shear and Average Velocity Semi-Analytically, Ref.[11]

neglects the narrow slot of the groove neck and combines the Laplace-Young and simplified Navier-Stokes equation to find the interfacial radius of curvature for a given radial heat input. A finite element grid seen in Figure 1.8 is produced to find the temperature distribution. A relatively large number of elements are placed in the groove neck due to the high heat flux near the micro-region which itself has a high heat flux and evaporation rate. The temperature domain in Figure 1.8 is analogous to the velocity domain and so a similar approach can be used to obtain a solution.

\subsection{Heat Transfer Limits}

Associated with heat pipes are various physical phenomena that limit the amount of heat that can be transferred, Ref.[14]. These limits are seen in Figure 1.9 as a function of temperature. 


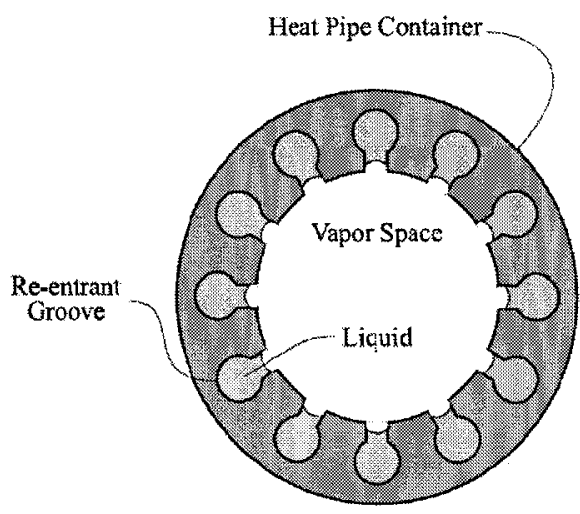

Figure 1.7: Re-Entrant Axially Grooved Heat Pipe Cross-Section, Ref.[9]

\subsubsection{Capillary Limit}

When the capillary structure is no longer able to sustain cyclic flow, the capillary (a.k.a. wicking) limit has been reached. The capillary limit is considered most likely to be reached first with the axially grooved heat pipe because of its large open grooves compared to a meshed design.

Prediction of the capillary limit based on a balance of surface tension and pressure effects along a heat pipe was discussed in Ref.[15] for simple geometries. This was carried one step further in Ref.[16] where the process mentioned in Ref.[15] was automated using a Visual Basic code. The ability to analyze an arbitrary geometry was still lacking and no comparison with experimental work was given. This led to the development of a code that would accept an arbitrary geometry. The geometry of a tested heat pipe could be input to the code and the numerical results could be compared with experimental results. 


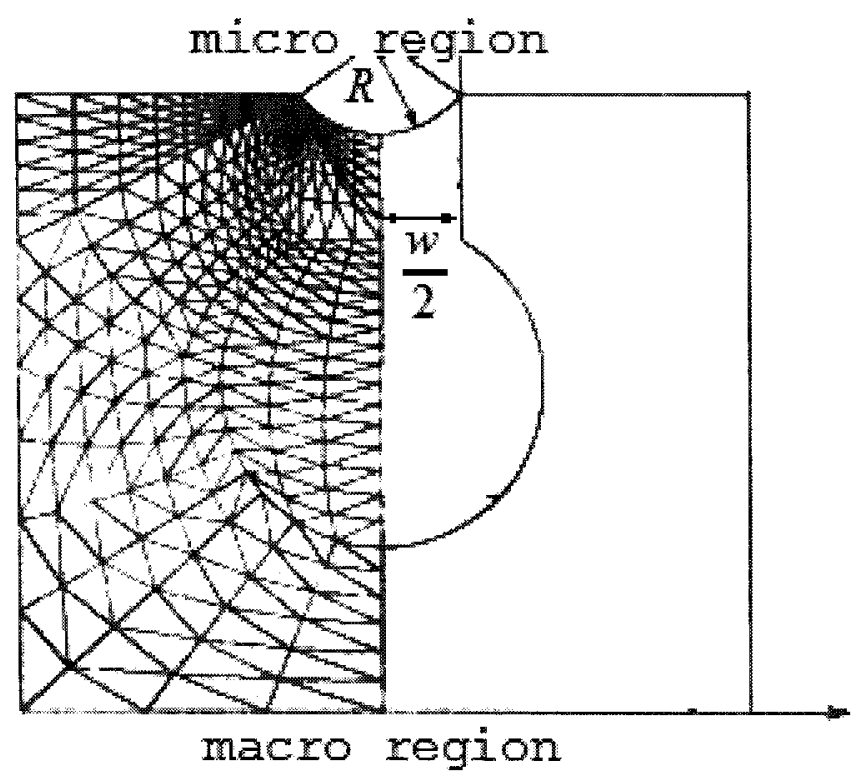

Figure 1.8: FEM Grid for a Re-Entrant Groove, Ref.[13]

\section{$1.9 \quad$ Arterial Heat Pipes}

A typical arterial heat pipe cross-section is seen in Figure 1.10. Grooves can be placed on the wall to aid the liquid in climbing to the evaporator as seen in Figure 1.10. A mesh can be placed in the artery to aid in motion of the liquid. Work on arterial heat pipes has been primarily a Russian endeavour since Americans found too much vapour in the artery and refused to readily include them in their spacecraft, Ref.[17]. Arterial heat pipe development has nevertheless continued and proven valid enough for use of arterial heat pipes as radiators on the International Space Station, Ref.[18]. Experiments were conducted on Space Shuttle STS-43 to test arterial heat pipe radiators, Ref.[19]. The use of heat pipes as radiators in orbit provide redundancy in case of micrometeoroid impact compared to flow-through radiators. Piercing a flow-through radiator would cause total fluid evacuation, while piercing one among an array of arterial heat pipe radiators would only cause partial damage. 


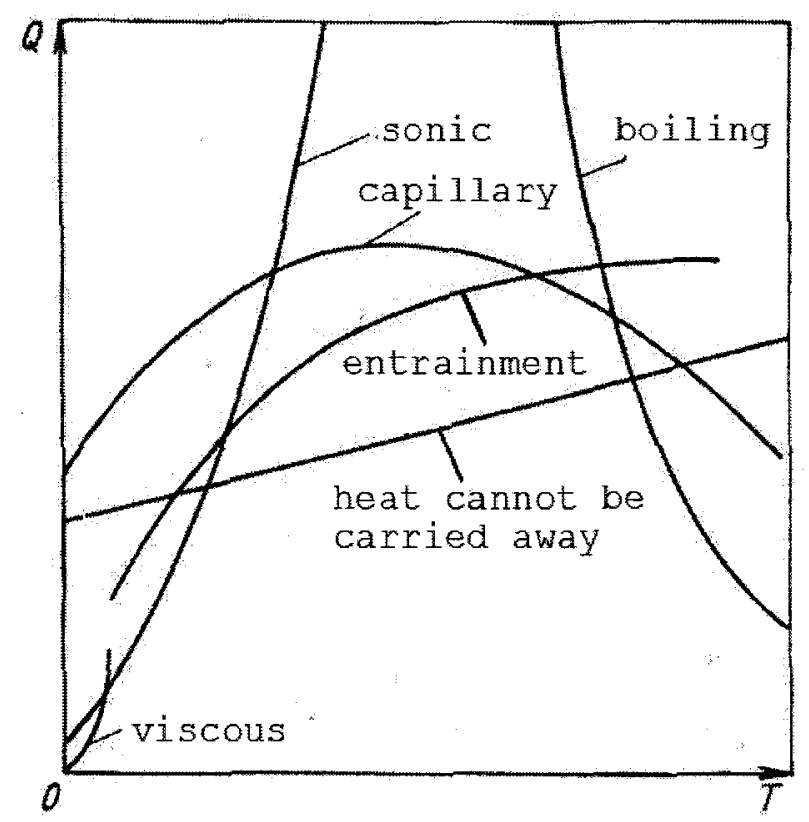

Figure 1.9: Heat Transfer Limits Encountered with Temperature, Ref.[14]

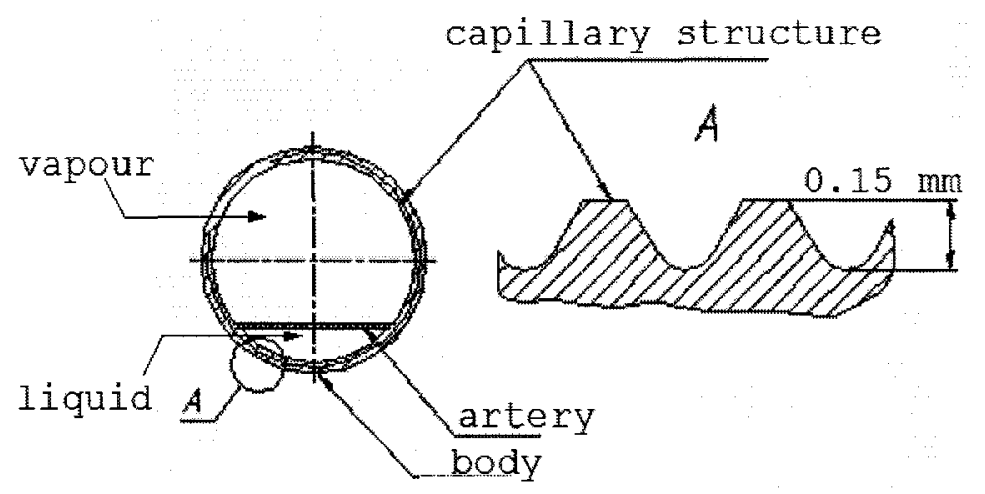

Figure 1.10: Typical Arterial Heat Pipe Cross-Section, Ref.[17] 


\section{Chapter 2}

\section{Experimental Investigation}

\subsection{Test Setup Summary}

Two ammonia charged u-shaped heat pipes with different geometries to separate liquid and vapour were tested. The first heat pipe tested was an axially grooved heat pipe which will hereafter be referred to as AGHP. The second heat pipe tested was an arterial heat pipe (AHP). Experiments were performed to determine the performance of individual heat pipes with varying heat source, heat sink, and gravitational inclinations.

The test matrix included heat inputs of $(0,40,80,120,160) \mathrm{W}$ and coolant sink temperatures of $(5,8,10,12,15,16,20){ }^{\circ} \mathrm{C}$. Dryout tilt testing with $5 \mathrm{~W}$ input power and $5{ }^{\circ} \mathrm{C}$ sink was performed on both heat pipes with heat pipe position tilt increments of $1^{\circ}$. Horizontal dryout testing of both heat pipes with $5^{\circ} \mathrm{C}$ sink was performed using a more powerful heater than the one used to perform all other tests. 


\subsection{Experimental Equipment}

\subsubsection{General Setup}

All testing was performed within the Carleton University Heat Pipe Laboratory in room 2233 MacKenzie Building under ambient pressure and temperature. Both heat pipes were received from TAIS Thermal Systems Design and Manufacturing located in Russia. The AGHP setup without insulation can be seen in Figure 2.1. A view of the AHP test setup with insulation can be seen in Figure 2.2. The test setup can be seen schematically with labelled components in Figure 2.3.

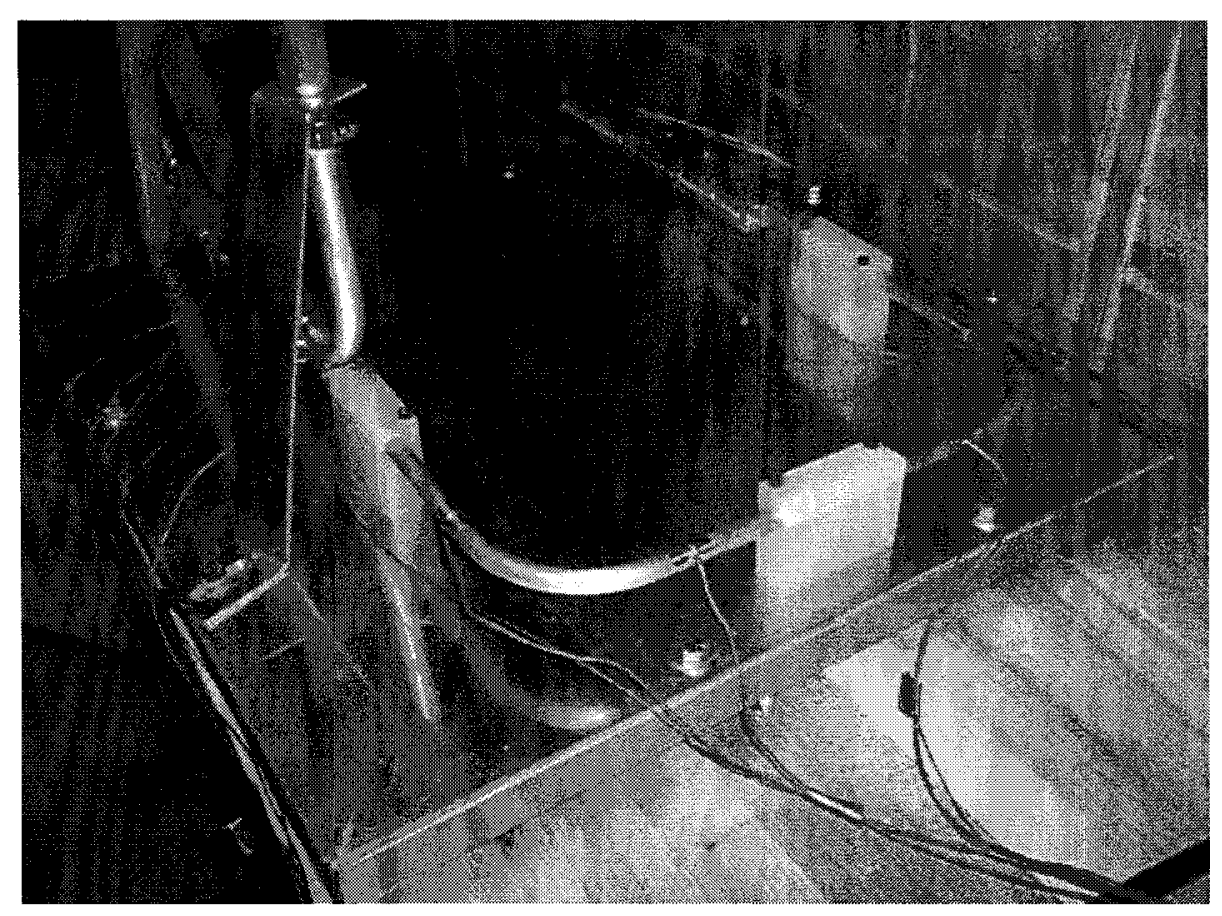

Figure 2.1: AGHP Setup without Insulation

The heat pipes were connected to subsystems that influenced or directly manipulated and recorded state variables. These subsystems included the heating, cooling, 


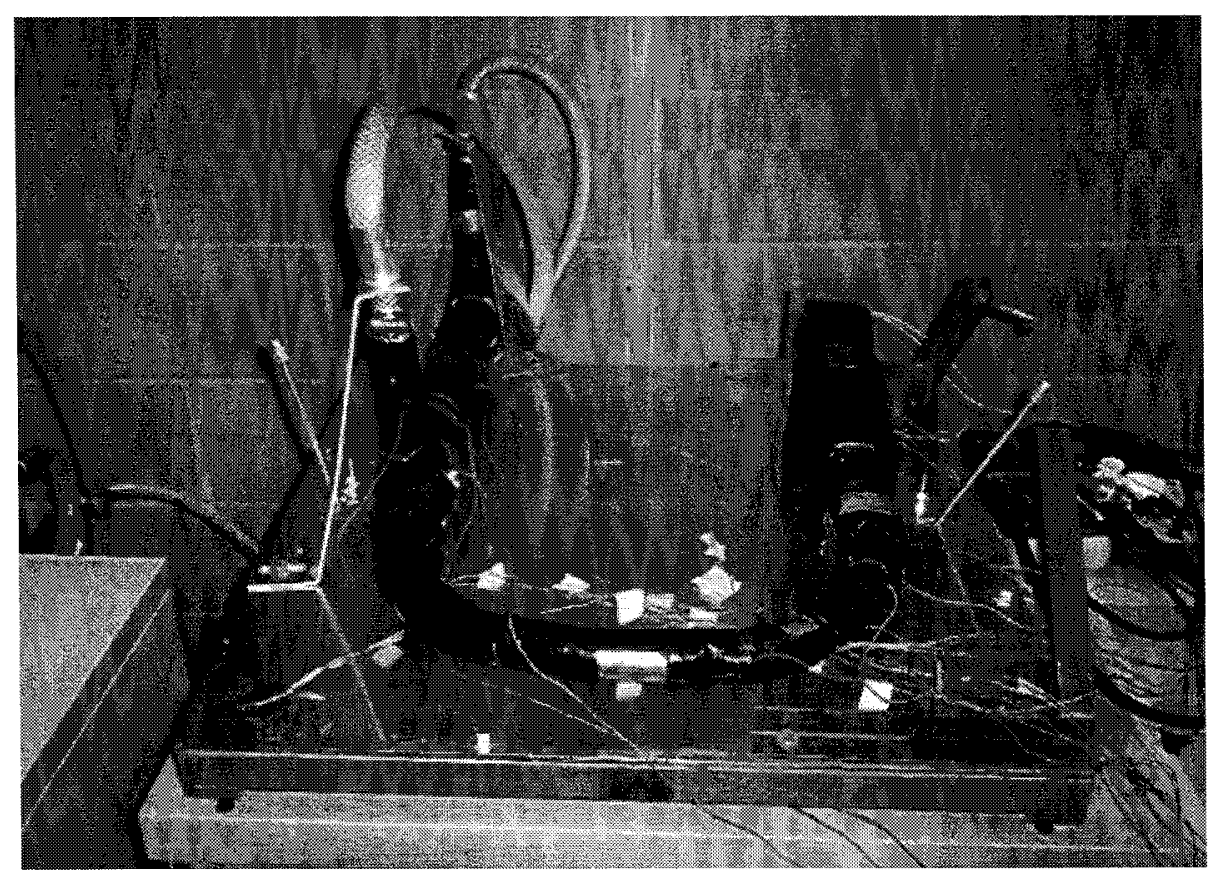

Figure 2.2: AHP Setup

insulation, support, inclination, and data acquisition. The heating and cooling systems were used to provide constant source power and coolant sink temperature, respectively. The insulation and support systems were used to create conditions that could allow reasonable assumptions for analysis and modelling purposes. The inclination system was used to apply desired gravitational body forces on the working fluid. The data acquisition system was used to sample and record temperature data of the heat pipes and various subsystems. A more elaborate exploration of the various subsystems follows.

\subsubsection{Heat Pipes}

The AGHP and AHP are shown schematically in Figure 2.4 and Figure 2.5, respectively. The AGHP is a $12.5 \mathrm{~mm}$ diameter 6063 aluminum alloy extrusion with a cap 


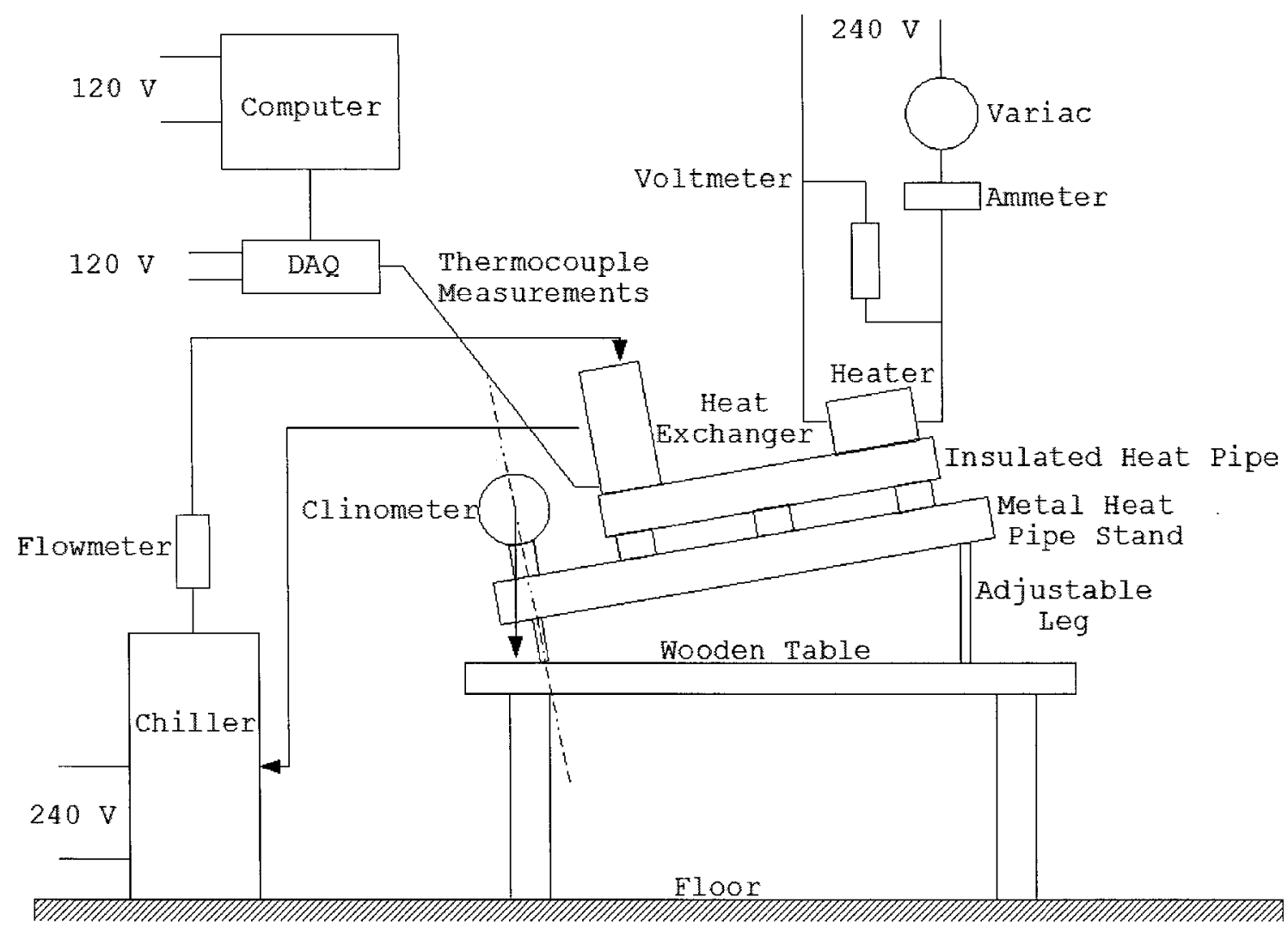

Figure 2.3: Test Setup Schematic

made of aluminum alloy. The AHP is made from $12 \mathrm{~mm}$ diameter stainless steel tubing of $0.5 \mathrm{~mm}$ thickness. The internal surface of the body consists of a capillary groove structure designed to help fluid to travel from the liquid filled stainless steel mesh artery to the vapour groove. The mesh was spot welded to the casing. The cap is made of stainless steel. Aluminum contact flanges were soldered to the AHP to allow for the mounting of the heater and heat exchanger. 


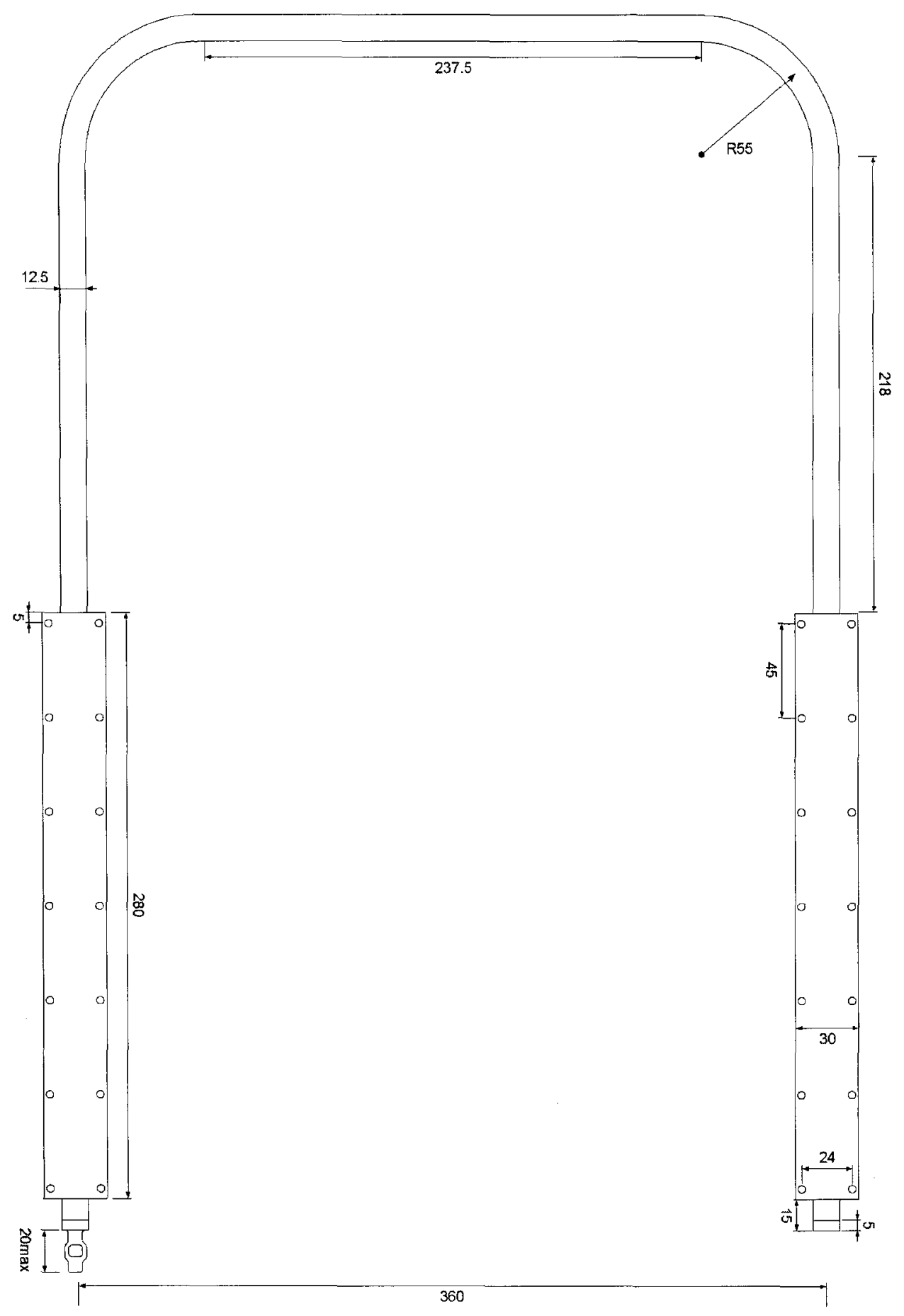

Figure 2.4: AGHP Drawing (all dimensions in $\mathrm{mm}$ ) 


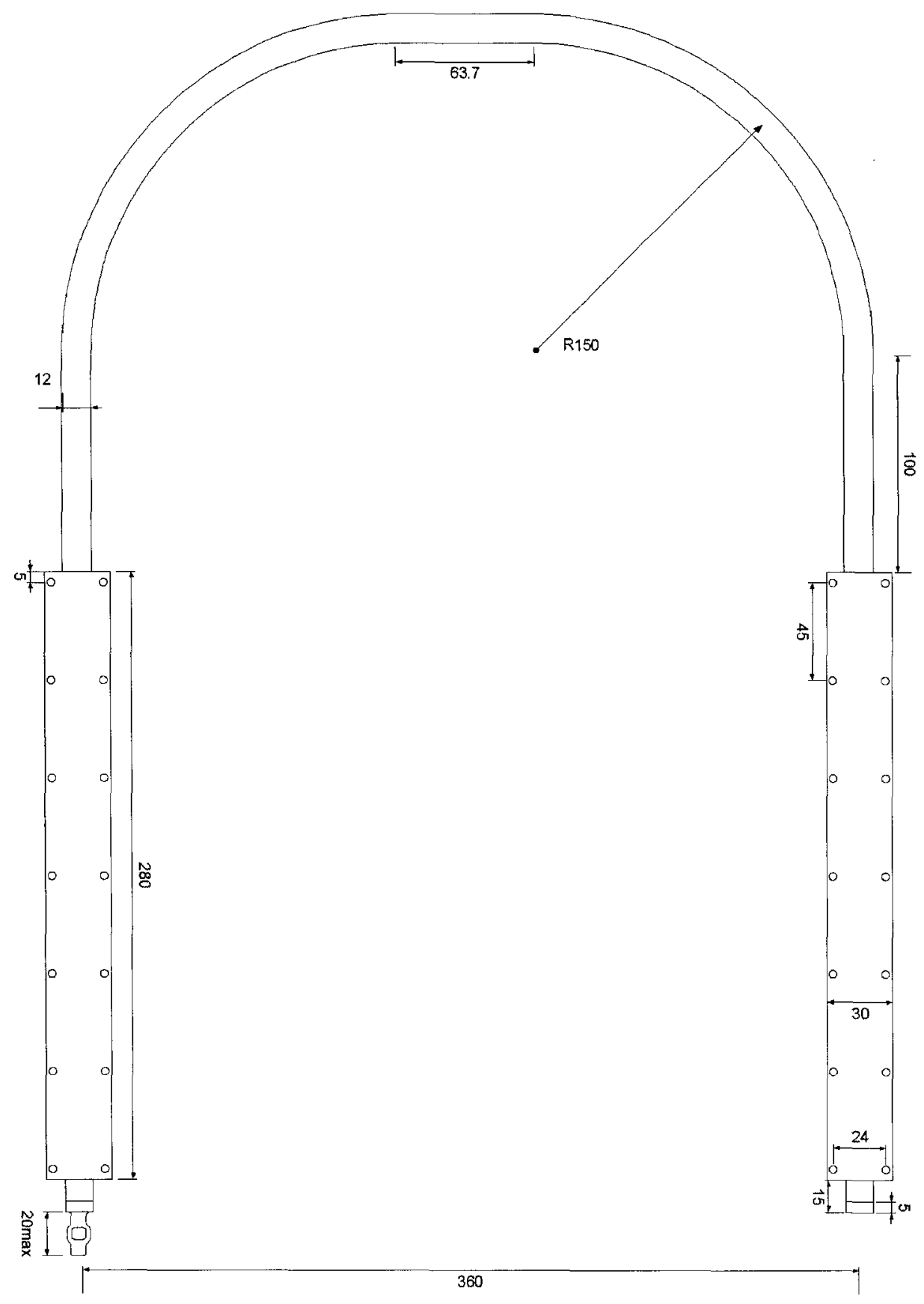

Figure 2.5: AHP Drawing (all dimensions in $\mathrm{mm}$ ) 


\subsubsection{Heating}

The heating system was used to vary the heat input to the evaporator. Two heaters were used to perform tests having different input requirements.

Electrical power was taken from an alternating $240 \mathrm{~V}$ source and passed through a variable transformer which was manually adjusted such that the product of a digital voltmeter and ammeter RMS display was within tolerance of the desired power input. Tolerance on the read power input compared to the target power input was taken to be $0.5 \mathrm{~W}$. The heating elements were assumed to carry a purely resistive load, thereby making the phase angle equal to zero and the actual power is simply the product of the voltage and current.

The first heater represented by Figure 2.6 consists of a rectangular aluminum plate designed to fit on flanges permanently attached the top and bottom of the evaporator. Heat is applied via glued film heaters and is guided towards the pipe with a $20 \mathrm{M} \Omega$ insulation resistance strip. Bolts were passed through every second column of heat pipe flange holes and every column of heater holes since the heater had with fewer holes than the flange.

An aluminum block casing for a $0.5 \mathrm{in}(12.7 \mathrm{~mm})$ diameter by $7.5 \mathrm{in}(190.5 \mathrm{~mm})$ long $1000 \mathrm{~W}$ cartridge heater was machined to fit the heater saddle for horizontal dryout testing since the original heater could not provide enough heat to cause dryout of either heat pipe given the maximum input current of $9 \mathrm{~A}$ stated by the manufacturer. Thermal joint compound was placed between the cartridge and casing. Bolts were fastened to create a tight fit between the heater cartridge and the aluminum casing block as seen in Figure 2.7. Given a tighter fit exists when the bolts going through the slit are tightened, the converse allows for relatively easy removal of the 


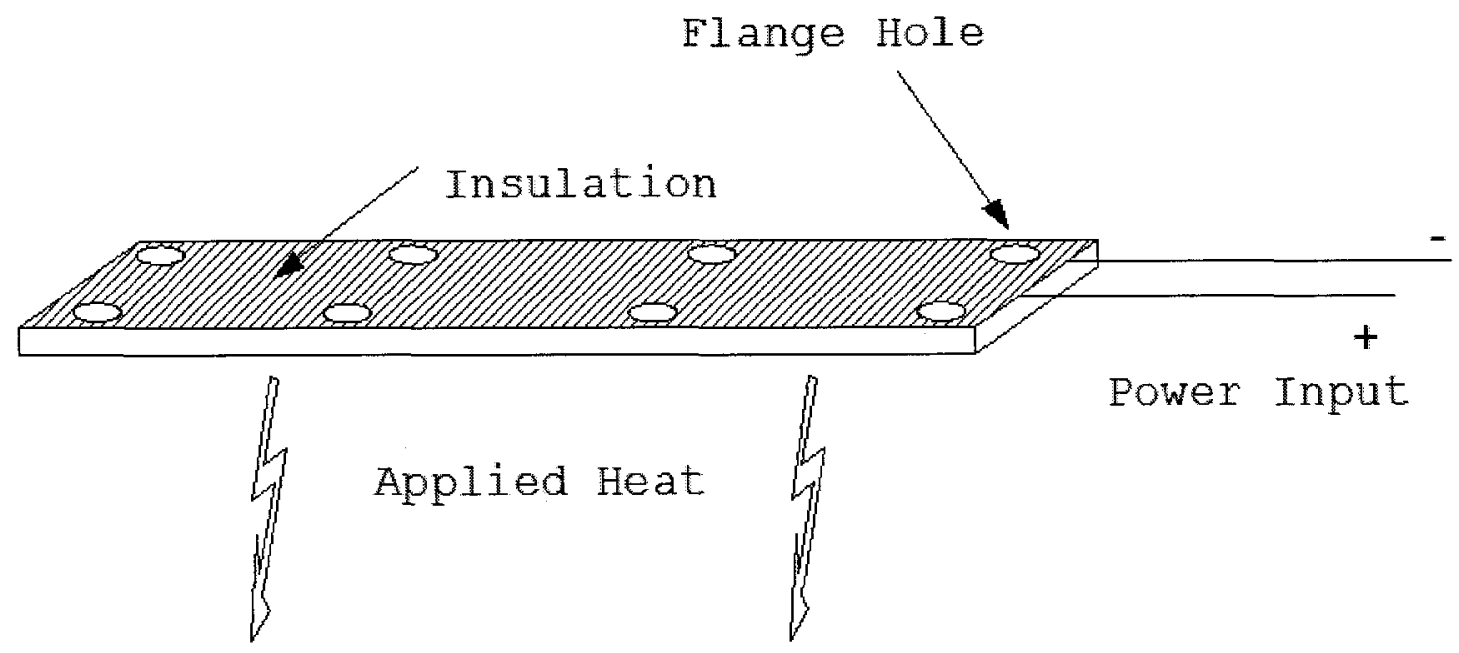

Figure 2.6: Low Power Film Heater

cartridge heater in comparison to a design with a single hole and no slit. Two plastic legs were affixed on two diagonally furthest bolts in order to support the weight of the heater that would otherwise bend the attached heat pipe (not shown in Figure 2.7). With the evaporator flange longer than the cartridge heater, two cylindrical end pieces of equal length were placed on either end of the cartridge such that the middle of the cartridge corresponded to the middle of the evaporator. A torque of 1.7 Nm was applied to hexagonal head flange bolts using a 0.25 in $(6.35 \mathrm{~mm})$ drive Armstrong dial torque wrench as per the limit specified by the manufacturer Ref.[20] in order to avoid damaging the heat pipe connection flange. The heater was placed above the evaporator for most of the testing of both heat pipes. A comparative test with the heater below the AHP evaporator was performed to observe the effect of artery location with respect to heater location. 


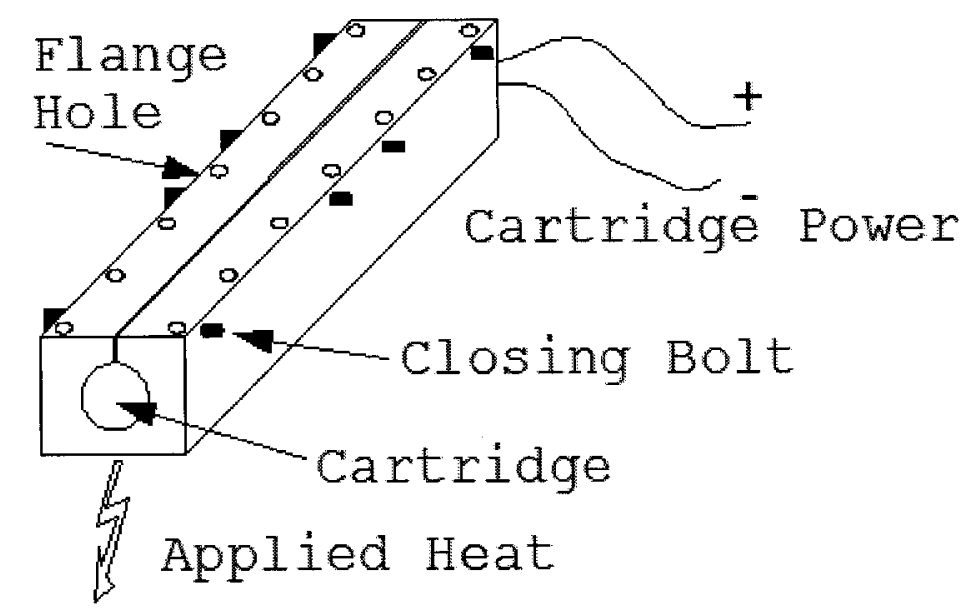

Figure 2.7: Cartridge Heater Block Cross-Section

\subsubsection{Cooling}

The cooling system was used to provide a heat sink at the condenser. The coolant was circulated using a Lytron-RC011 chiller with a 1.8 GPM (6.8 LPM) capacity positive displacement pump. The chiller refrigerant was R-134a. The volumetric flow rate was measured using a Omega FL-2068 tapered acrylic flowmeter seen in Figure 2.8. The average flow rate was around 6.7 LPM.

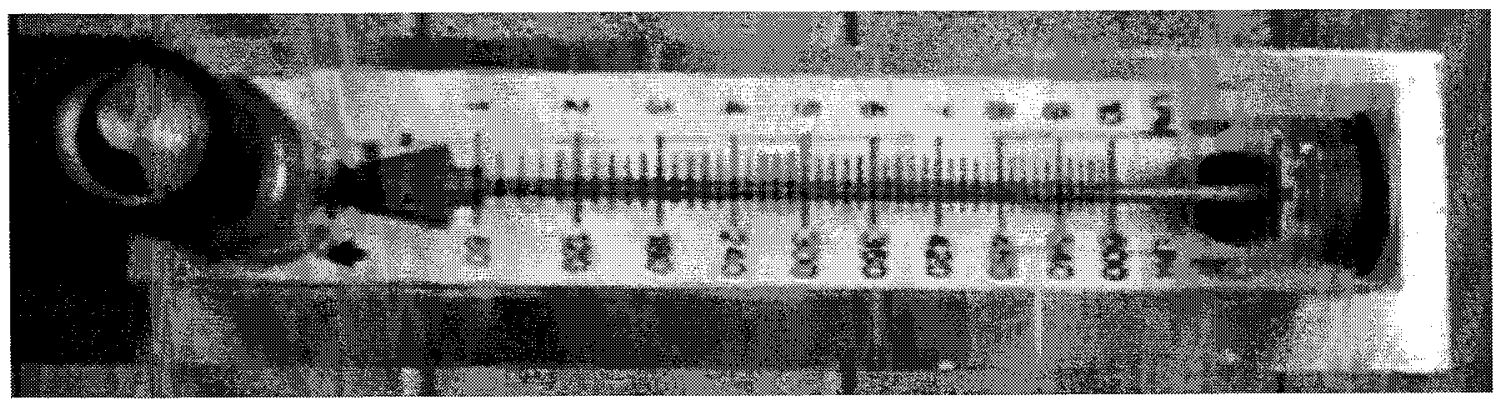

Figure 2.8: Omega FL-2068 Flowmeter 
The heat exchanger working fluid consisted of $70 \%$ water and $30 \%$ laboratory grade ethylene glycol by volume as suggested by a Lytron engineer through correspondence. Ethylene glycol was used along with water to avoid the formation of ice particles within the flow circuit that could potentially damage the pump when operating coolant at $5{ }^{\circ} \mathrm{C}$. Automotive antifreeze was not used because the inherent rust inhibitors could cause premature failure of the pump seals, Ref.[21]. The heat exchanger was connected to the condenser saddle with thermal joint compound. Fastening with flange bolts was done in the same manner as with the heater. The heat exchanger was placed exclusively above the condenser due to space restrictions imposed by the testing platform.

\subsubsection{Insulation and Support}

The insulation system consisted of one layer of polyolefin insulation typically found in domestic plumbing copper piping circuits. The insulation was used around the heat pipe, heater, and heat exchanger. The input and output 0.5 in $(12.7 \mathrm{~mm})$ inner diameter plastic coolant lines between the chiller and heat exchanger were not insulated since the high mass flow rate of the fluid gave it little time to change temperature while travelling towards the heat exchanger. Temperature change measured between the chiller and heat exchanger input with decreased mass flow rate showed an increase in temperature deemed unacceptable if one wishes to use the chiller temperature as the sink temperature to define a test. Testing at a low coolant mass flow rate would preferably require short and insulated coolant lines. The downside to a shorter connection is transmittance of chiller induced vibrations more easily to the heat pipe. A damping system could be connected to resolve this issue. 
The heat pipes were fixed to a level metal table using three polypropylene blocks. A half-circular groove was milled in all blocks and end caps to contour the heat pipes. The end caps were fastened to the blocks using hexagonal head screws. High strength two-sided tape was used to fix the blocks to the heat pipe stand. When switching from one heat pipe to another, one of the blocks had to be moved since the heat pipes had different dimensions. Support brackets connecting the heat exchanger to the metal table were used to reduce vibrational loading from the refrigeration unit and stress on the connected heat pipe. Gravitational body forces due to the heat pipe being unintentionally placed at an angle were avoided by calibrating four adjustable legs of the heat pipe stand with two levels perpendicular to one another in a plane parallel to the heat pipe. Electrical charging of the heat pipe that could potentially affect thermocouple readings was avoided by grounding the heat pipe using an outlet ground inside the laboratory.

\subsubsection{Tilting Mechanism}

Tilting the heat pipe stand through small angles $\left(1^{\circ}\right.$ changes to a maximum total of about $25^{\circ}$ ) was performed using the setup seen in Figure 2.9 and with more detail in Figure 2.10. The threaded rod acts as the third leg of a tripod which can be adjusted. The two remaining legs act as fixed contact pivot points between the table and heat pipe stand. The heat pipe stand is raised and lowered by using a socket wrench to rotate the adjustable threaded rod attached to a threaded hinge which is in fixed to the heat pipe stand. Various methods for determining the tilt angle value were explored. 


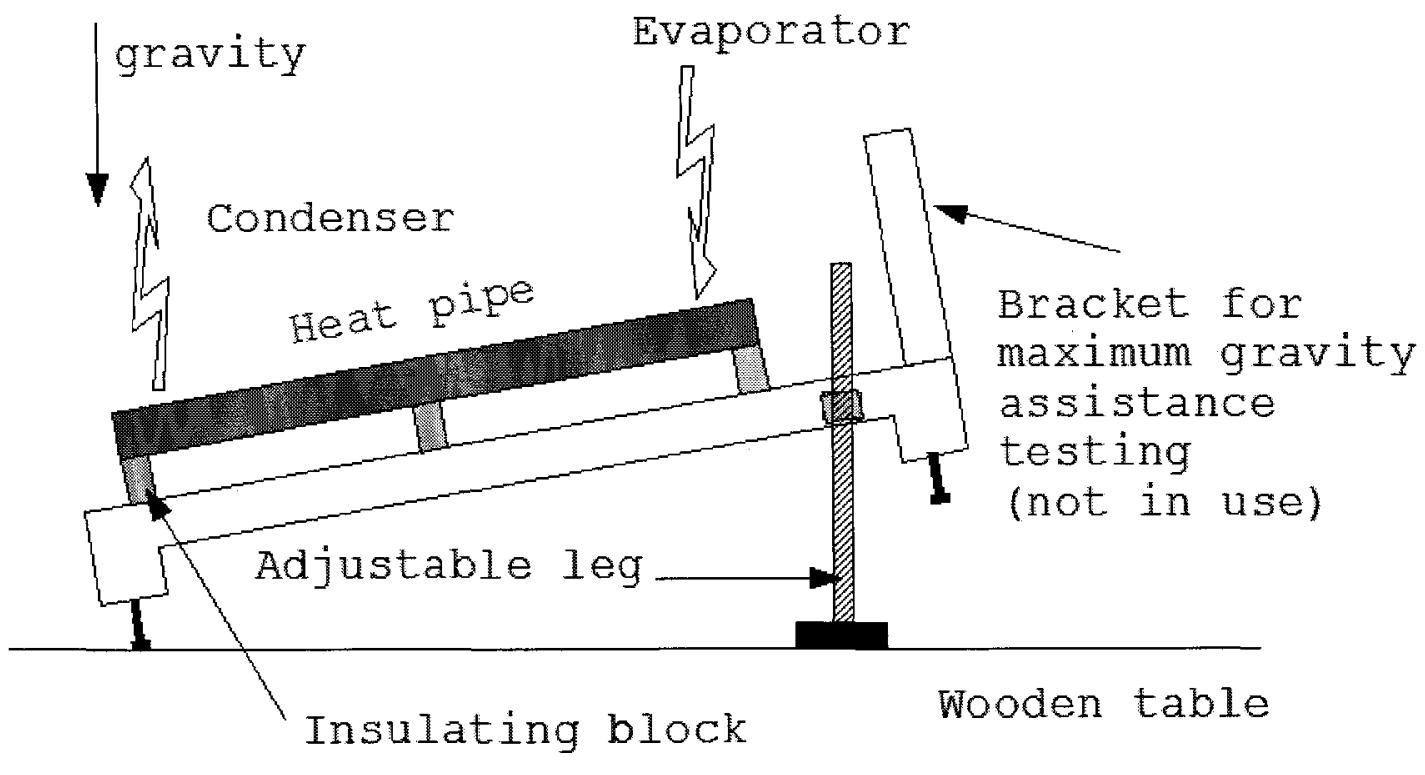

NOT TO SCALE

Figure 2.9: Tilting Mechanism

\section{Direct Angle Measurement}

The first attempt was simply to trace the line made by an edge of the heat pipe stand and a rectangular metal plate placed on a horizontal table. A regular protractor was then used to get the angle. This method proved to be time consuming and given to poor precision.

\section{Plumb Bob}

Another method involved the use of a plumb bob hung about 30 in (762 mm) above the heat pipe stand as seen in Figure 2.2. The horizontal distance travelled by the plumb bob between no inclination and the point of desired inclination was related to a tilt angle using the position of the stand pivot point and the original pivot point of the plumb bob line. These geometrical relations were made into a function by using a 
computer program that would take the horizontal distance travelled as an input and give the tilt angle as an output. If specific tilt angles were required prior to testing, a sheet of paper with "angle lines" indicating the desired tilt angle was placed under the plumb bob such that its path would be perpendicular to the lines. The plumb bob method gave sufficient accuracy, but its major downfall was the time elapsed between physically changing the tilt angle and knowing the value of the change. The reason for this large time period required was that energy input into the heat pipe stand via rotation of the lifting screw would cause the plumb bob to oscillate for a rather long time before stopping and allowing for the angle to be deduced. The time of oscillation was reduced by imposing friction on the plumb bob by raising the stand on which the lined paper was located. When the plumb bob was almost still, the measurement stand was lowered such that it would no longer touch the end of the bob. This minute distance between the angle line and the plumb bob also reduced the error associated with judging how close to directly overhead the plumb bob was to the angle line. Another method to reduce the time lost to oscillation could have been to place the plumb bob in a water bath with a transparent bottom so the angle line sheet could be read. This method was not attempted because the plumb bob method was abandoned altogether due to its complexity with little benefits.

\section{Laser Dot}

Measurement of the tilt angle was also attempted using a laser pointer in place of the plumb bob. The difference in the function to obtain the angle from the horizontal distance lies in the fact that the laser does not point downwards constantly as would the plumb bob due to gravity. An awkward test setup also had to be made in order to achieve the same resolution as the plumb bob due to the different inherent functions. This method was abandoned because its complexity grew beyond reason. 


\section{Clinometer}

The clinometer seen in Figure 2.11 was eventually chosen as the best tilt angle measurement device compared to the setups described previously. The angle is read directly from the display without having to use complicated functions, the $0.5^{\circ}$ accuracy is considered acceptable, no time consuming oscillations are endured, and the instrument cost is relatively low.

\section{Right Angle Testing}

Testing with the maximum assistance from gravity was done by rotating the heat pipe stand $90^{\circ}$ and securing it via two brackets (one is seen in Figure 2.9) bolted to the metal heat pipe stand and the wooden test table. Washers were used as shims between the brackets and the table to make the face of the heat pipe stand nearly parallel to gravity.

\subsubsection{Data Acquisition}

The data acquisition system contained 23 Omega T-type thermocouples. The thermocouple breakdown consisted of 7 for each the evaporator and condenser, 6 for the adiabatic section, 2 for the coolant input and output, and 1 for the ambient room temperature. The ambient temperature thermocouple was fixed to the heat pipe stand because its size gives it a high thermal mass useful for the filtering of temperature changes associated with light air currents that would not affect the heat pipe significantly due to its insulation. The general thermocouple locations for both heat pipes is shown in Figure 2.12. In the areas where the heater or heat exchanger made contact with the heat pipe, thermocouples were placed on the heat pipe saddle unoccupied by the heat transfer device. Heat resistant aluminum tape commonly 
used to seal warm air ducts was used to fix the thermocouples to the heat pipe body. Muffler repair tape was used to fix thermocouples near the evaporator since conventional tape would sometimes disconnect due to overheating. The muffler tape was not used for all thermocouples because of its higher price and difficulty of removal. The heat pipes were cleaned with isopropyl rubbing alcohol before connecting the thermocouples to obtain a consistent thermal conductivity between thermocouple locations and to help the tape adhere to the heat pipe surface.

Thermocouple data was sampled using a Keithley-2700 data acquisition system with internal self-calibration not requiring an ice bath. The thermocouple sample period was 23 seconds with equal time intervals between samples meaning that every second, a different thermocouple signal is recorded. Within thermocouple temperature text file matrices, each row represented one sample period and each column represented temperature at a unique thermocouple location.

Source and sink changes with respect to sample number (related to time) were manually recorded in another text file for further manipulation. The rows of these text file matrices represented one test setting and the columns contained the following test parameters: sample number, computer time (HH:MM:SS), input voltage, input current, input power, sink temperature, and tilt angle. Time at which each test setting changed was deemed important because the sampling frequency was not exactly 23 seconds when the period of roughly half an hour of testing was averaged. This is thought to be due to the time needed to reset and write the information to file. The discrepancy motivated the use of an independent timing device in case time critical data manipulation was to be performed. 


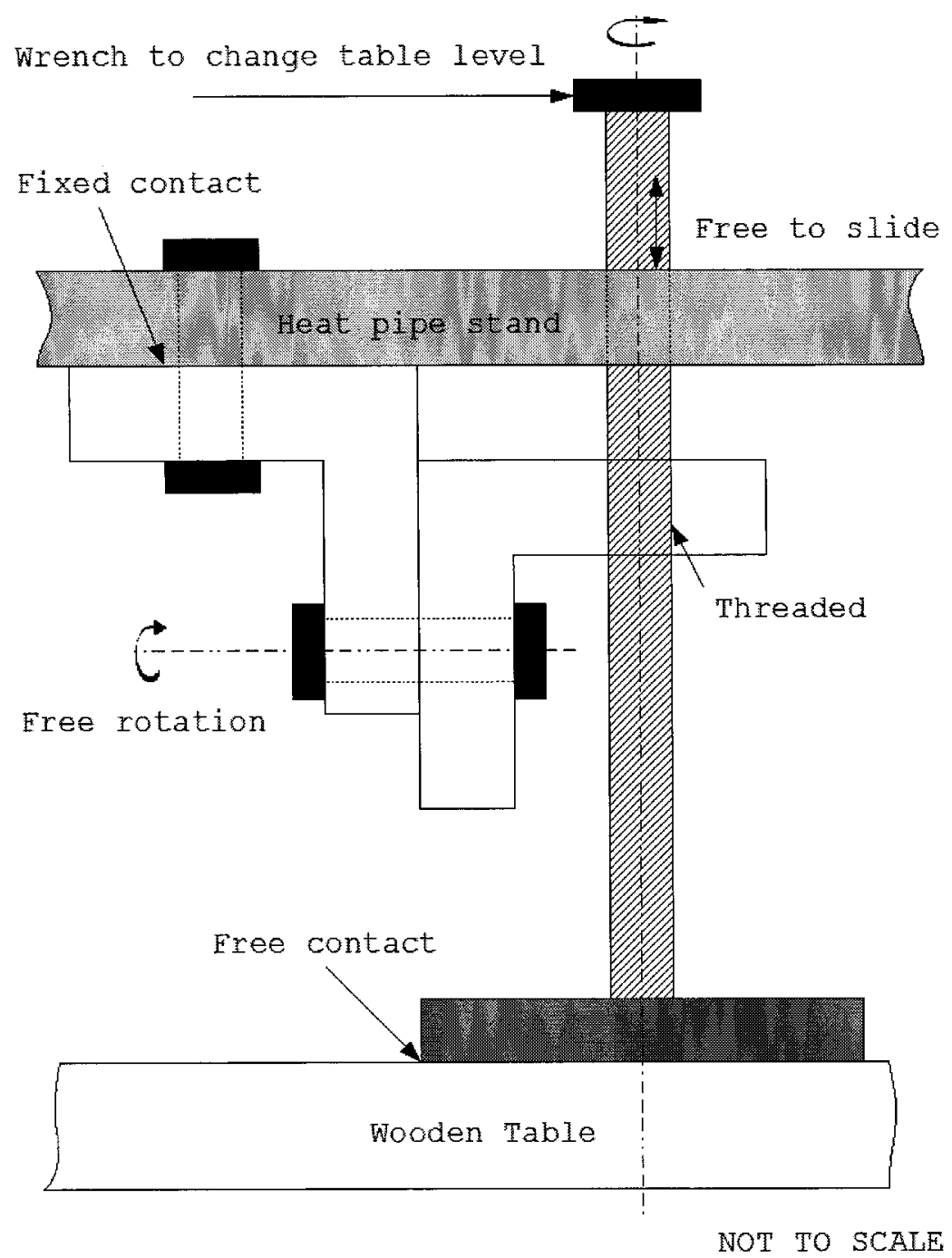

Figure 2.10: Heat Pipe Stand Adjustable Lift Mechanism 


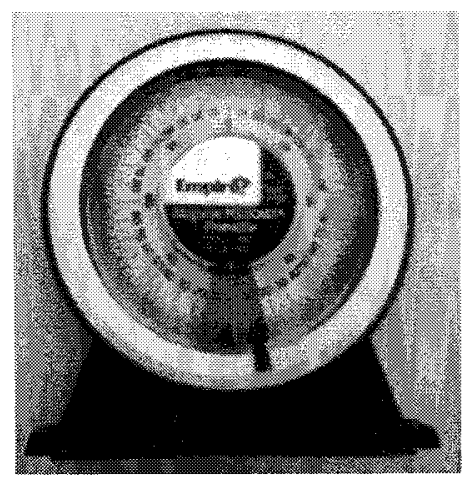

Figure 2.11: Clinometer used to Measure Tilt Angle

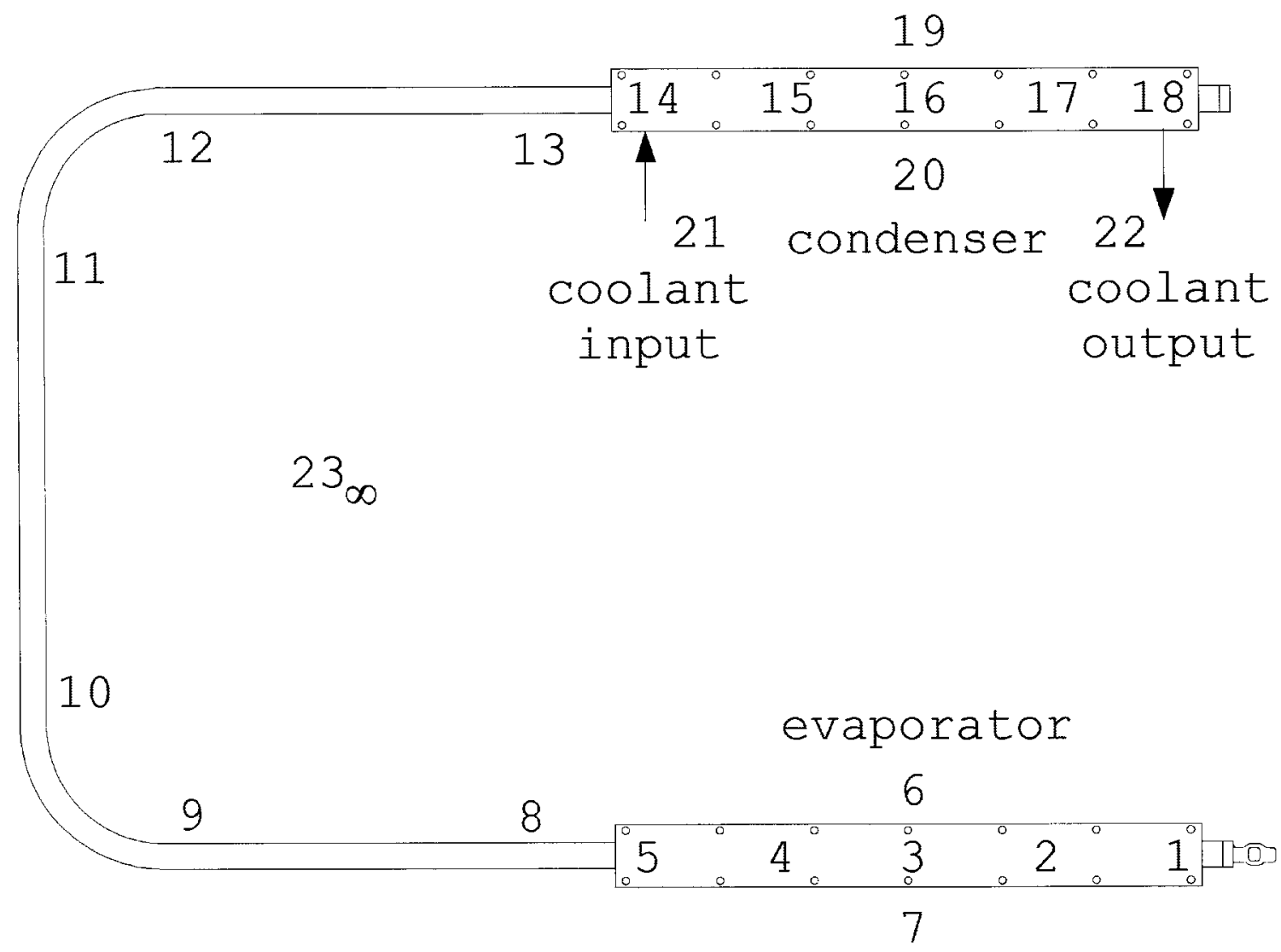

Figure 2.12: Thermocouple Locations 


\subsection{Experimental Process}

\subsubsection{Steady-State Response}

Steady-state conditions were desired for analysis. Steady-state was assumed at less than $0.1{ }^{\circ} \mathrm{C}$ variation over about ten minutes. This minute temperature fluctuation was observed by zooming into the graph created in real-time by the data acquisition software. The initial steady-state criteria imposed was $0.5^{\circ} \mathrm{C}$ over 30 minutes. It was observed that both heat pipe temperatures would reach steady-state either much before the prescribed 30 minutes or would oscillate about a nearly constant temperature indefinitely. A steady-state criteria should be found based on the observed performance of an individual heat pipe since an arbitrary choice could lead to a waste of time collecting static data.

\subsubsection{Transient Response}

The heat pipe transient response was deemed too dependent on subsystems and the coupled system was not investigated. The time for the heater block and especially the coolant to reach steady-state independent of the heat pipe was not negligible, so one could not draw conclusions solely for the heat pipe during the transient regime.

\subsubsection{Testing Performed}

Various tests were performed including: variable heat input with constant sink temperature, variable sink temperature with constant heat input, and the application of a varying gravitational body force until dryout. Gravity assisted tests with the condenser above the evaporator were also performed to compare with horizontal performance. 
Dryout testing of the AHP with the heater above the evaporator and furthest away from the artery was preformed. This is the worse case for performance because heat from the evaporator has to travel through the vapour groove to reach the arterial liquid before the necessary evaporation occurs in order to successfully complete the heat pipe cycle.

The heat exchanger was placed above the condenser. This condenser configuration makes it easier for the vapour to condense than if heat output also had to be transferred through the liquid artery. The combination with the heat exchanger below the condenser was not implemented because the test setup would have to be severely altered or a more fitting heat exchanger would have to be used.

The location of the heater and heat exchanger when testing the AGHP is not as important because of its angular cross-sectional symmetry. The majority of tests performed were without inclination and no intention of dryout. Source and sink were varied in a manner that can be seen in Figure 2.13.

The search pattern used to predict dryout for the AGHP was to increase power by $40 \mathrm{~W}$ until dryout was observed. The test was then repeated until the second last power setting was reached followed by power input increments of $10 \mathrm{~W}$. The AHP search pattern was changed from $40 \mathrm{~W}$ increments to $90 \mathrm{~W}$ increments when dryout did not occur well beyond the AGHP heat input limit. The test was repeated with finer final increments as with the AGHP.

Testing for hysteresis (the property that output depends on the history and direction of input) was done by returning to the original state once the largest heat input or lowest sink temperature was reached depending on whether heat input or 


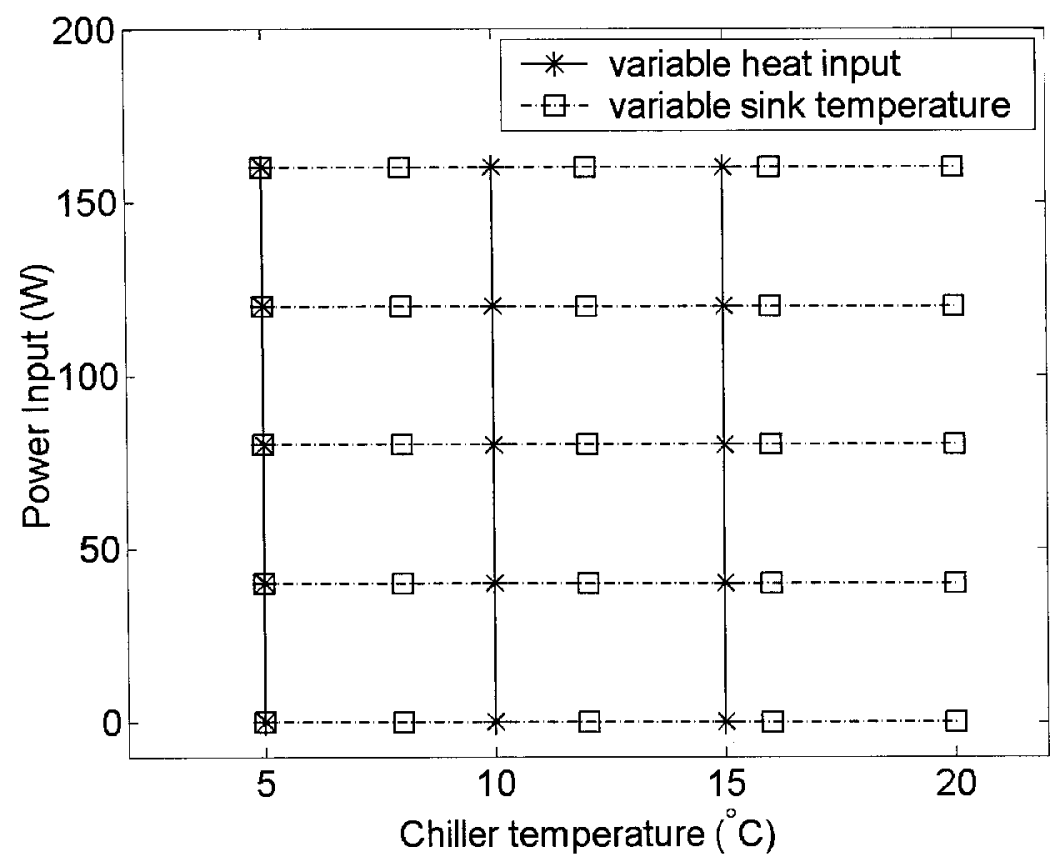

Figure 2.13: Variation of Heat Input and Sink Temperature

sink temperature was varied.

\subsection{Experimental Results}

\subsubsection{Temporal Temperature Variation}

The variation of temperature with time conveys as to whether or not steady-state has been reached. Each scan number represents roughly 24 seconds when comparing the number of scan numbers to the time elapsed for a typical test. There are only 23 thermocouple stations to read, but the total data acquisition switching and resetting takes around 1 second. 
Given that there are too many thermocouple readings to clearly display all the temperature information on a single graph, the average evaporator, adiabatic section, and condenser temperature are defined in Eqs. $(2.1,2.2,2.3)$ with numerical subscripts denoting positions in Figure 2.12:

$$
\begin{gathered}
T_{\mathrm{ev}}=\frac{1}{5}\left(T_{1}+T_{2}+T_{3}+T_{4}+T_{5}\right) \\
T_{\mathrm{ad}}=\frac{1}{6}\left(T_{8}+T_{9}+T_{10}+T_{11}+T_{12}+T_{13}\right) \\
T_{\mathrm{co}}=\frac{1}{5}\left(T_{14}+T_{15}+T_{16}+T_{17}+T_{18}\right)
\end{gathered}
$$

AGHP temperature versus time with constant sink temperature and variable heat input, and constant heat input and variable sink temperature is shown in Figures $2.14,2.15,2.16$ and Figures $2.17,2.18,2.19,2.20,2.21$, respectively.

AHP temperature versus time with constant sink temperature and variable heat input, and constant heat input and variable sink temperature is shown in Figures 2.22, 2.23, 2.24 and Figures 2.25, 2.26, 2.27, 2.28, respectively.

It is seen that both devices behave as heat pipes since the reaction to the application of heat at the evaporator is seen to occur nearly simultaneously along the heat pipe. This behaviour would not occur with a static heat transfer device such as a copper rod.

It is interesting to note the difference in response time between changing the heat input and coolant temperature. Changing the heat input is simply done by adjusting 


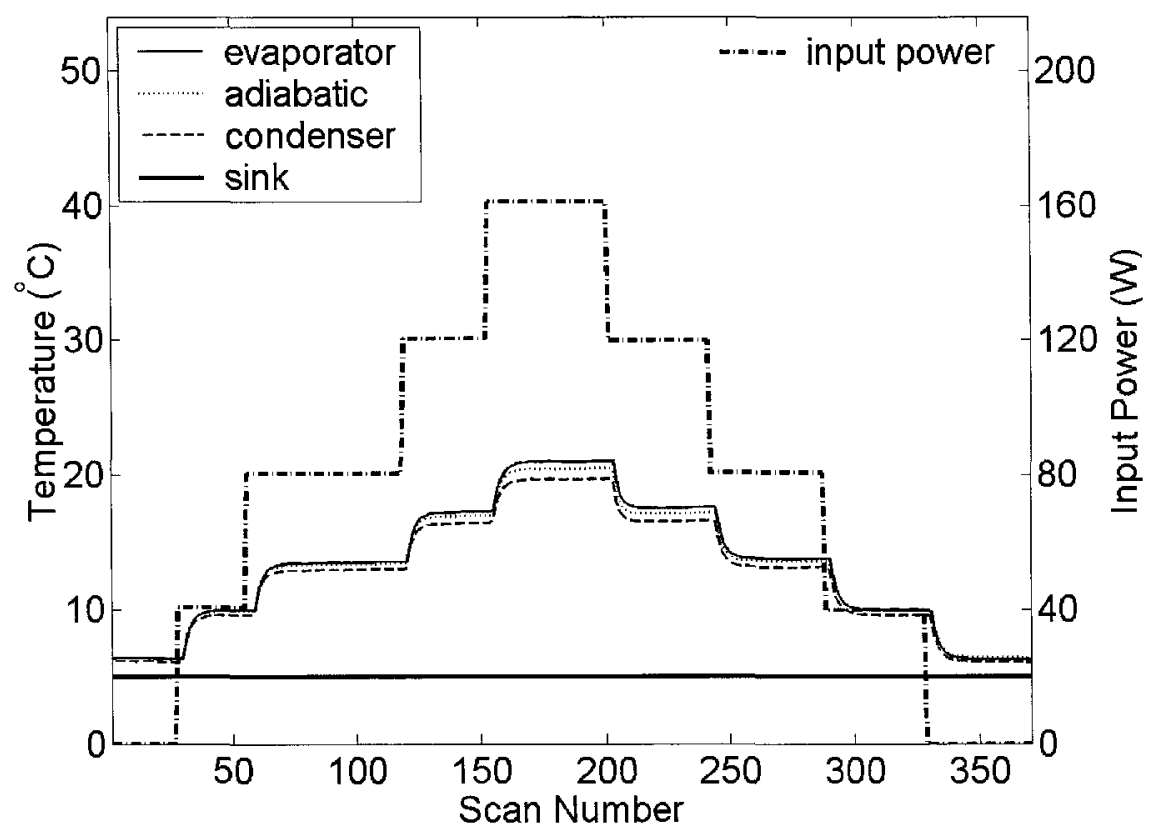

Figure 2.14: AGHP, Average Temperature, Sink $5{ }^{\circ} \mathrm{C}$

a variac which changes the power input nearly instantly. The power is run across a resistive heater connected to a metal bar that in turn heats the evaporator. Changing the coolant temperature on the other hand involves changing the chiller temperature setting which changes the coolant refrigeration temperature. The difference is the larger amount of time needed to change the temperature of the coolant which is related to the heat absorbed by the heat exchanger. Another interesting point relating to the change in coolant temperature is the overshoot or undershoot seen in the heat pipe temperature as one increases or decreases the set chiller temperature, respectively. This is believed to be caused by the chiller actually going beyond its target temperature before settling down. Therefore, the bumps seen in the variant coolant temperature profiles are not caused by the heat pipe physics. 


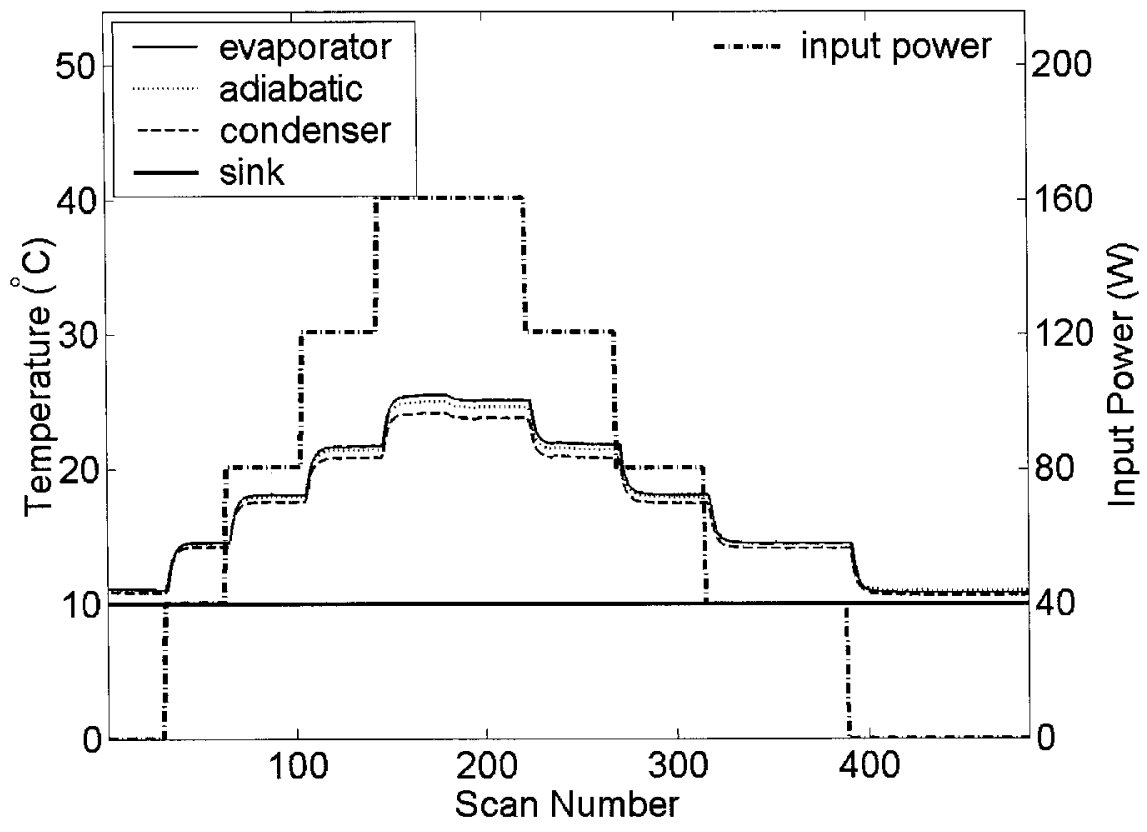

Figure 2.15: AGHP, Average Temperature, Sink $10^{\circ} \mathrm{C}$

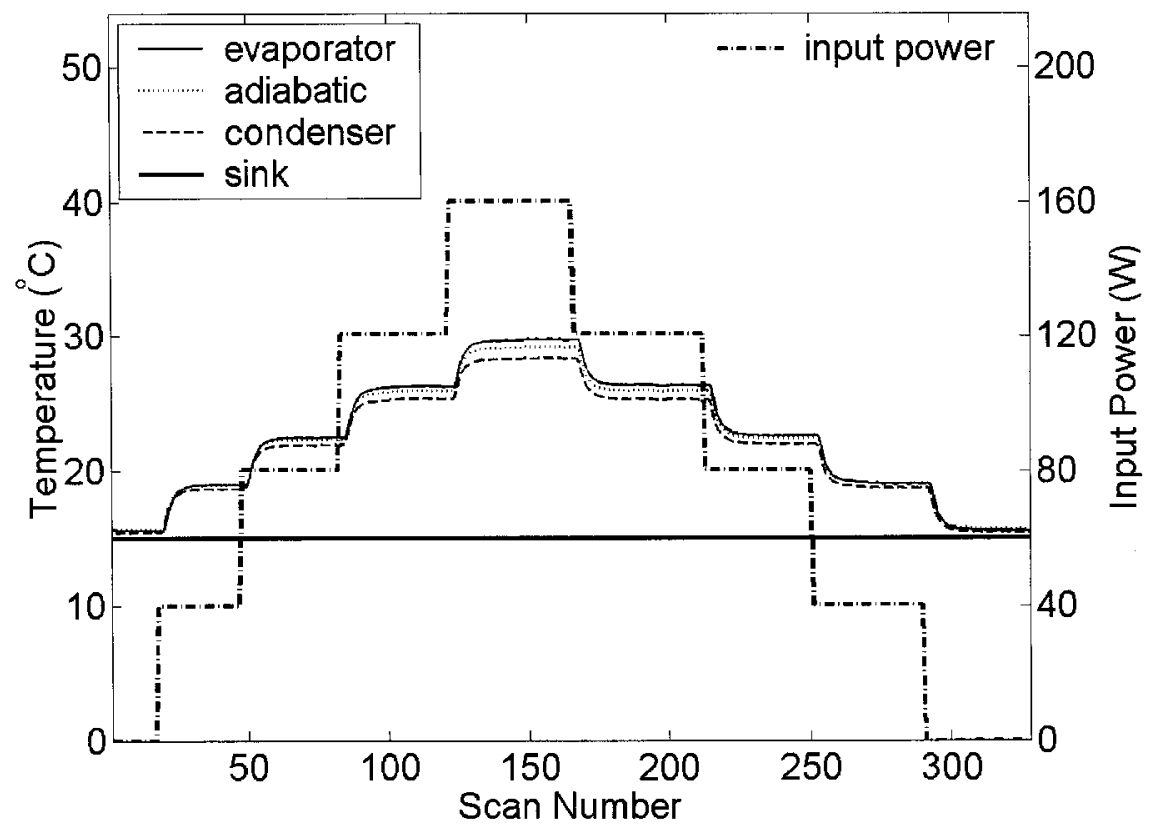

Figure 2.16: AGHP, Average Temperature, Sink $15^{\circ} \mathrm{C}$ 


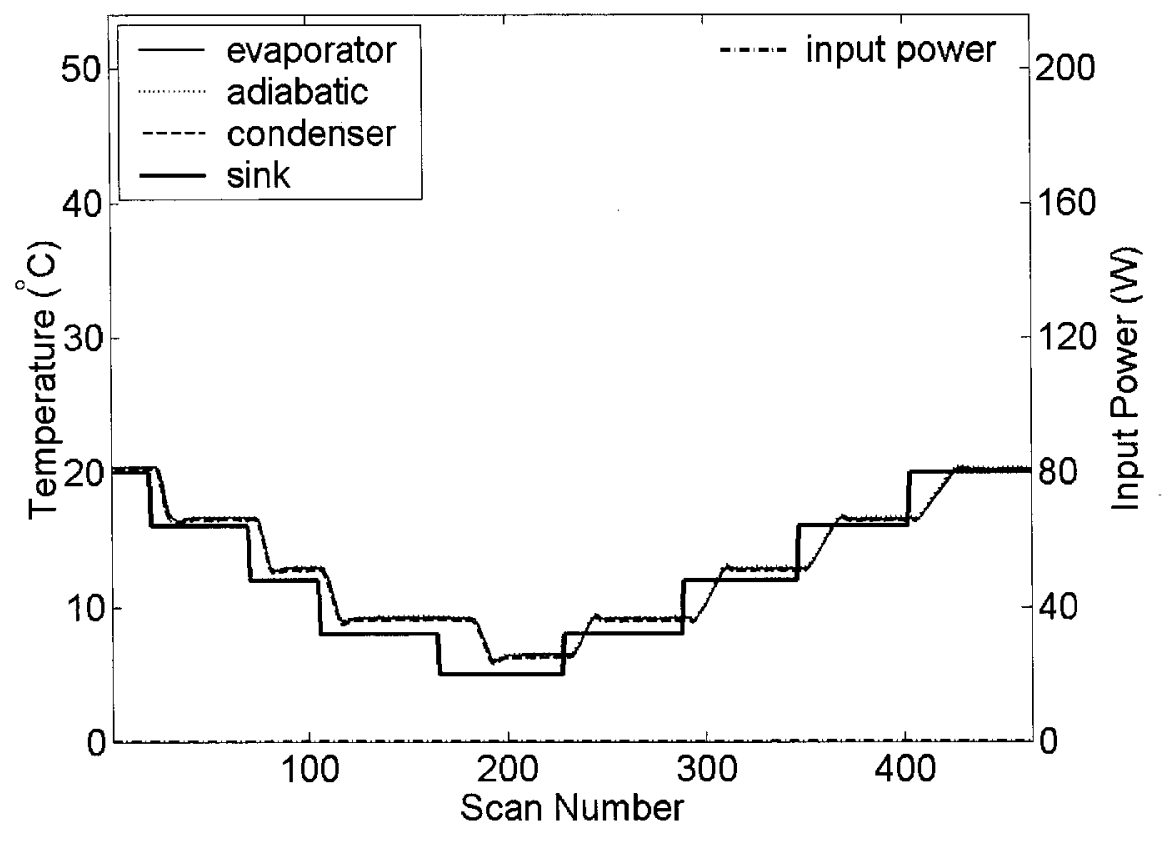

Figure 2.17: AGHP, Average Temperature, Source $0 \mathrm{~W}$

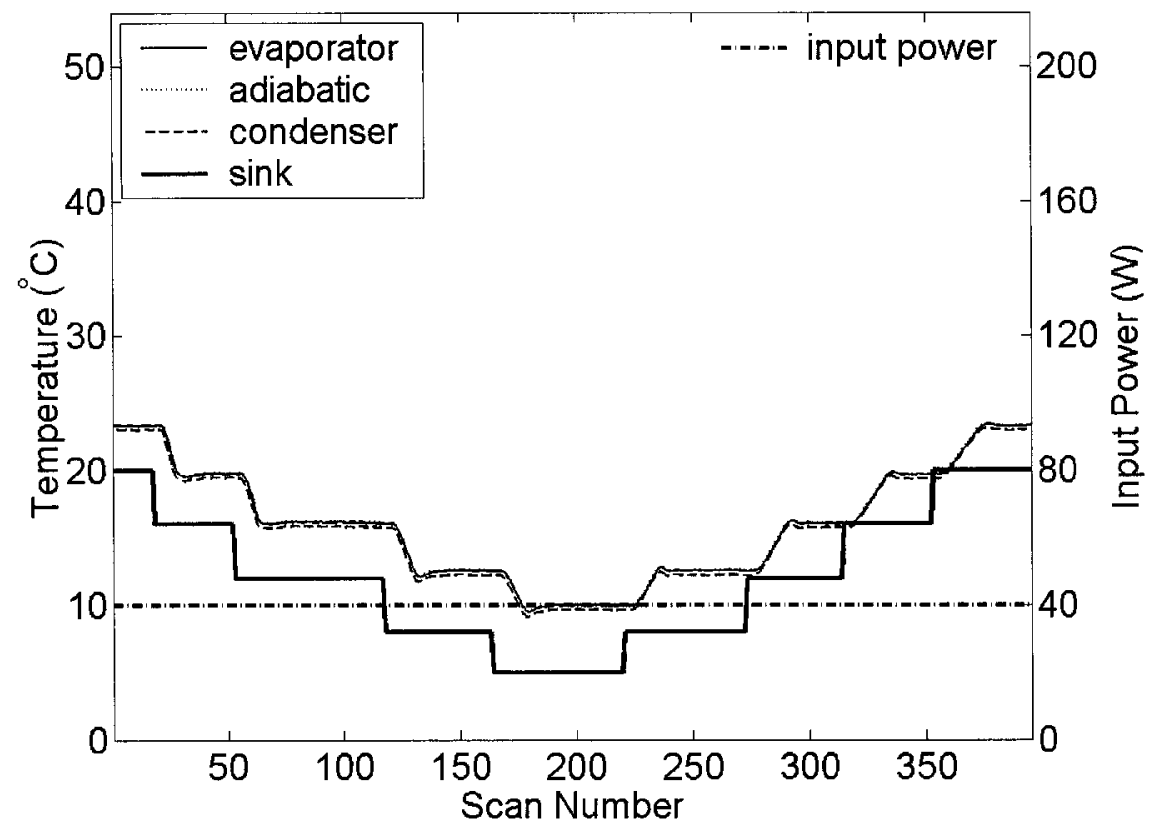

Figure 2.18: AGHP, Average Temperature, Source $40 \mathrm{~W}$ 


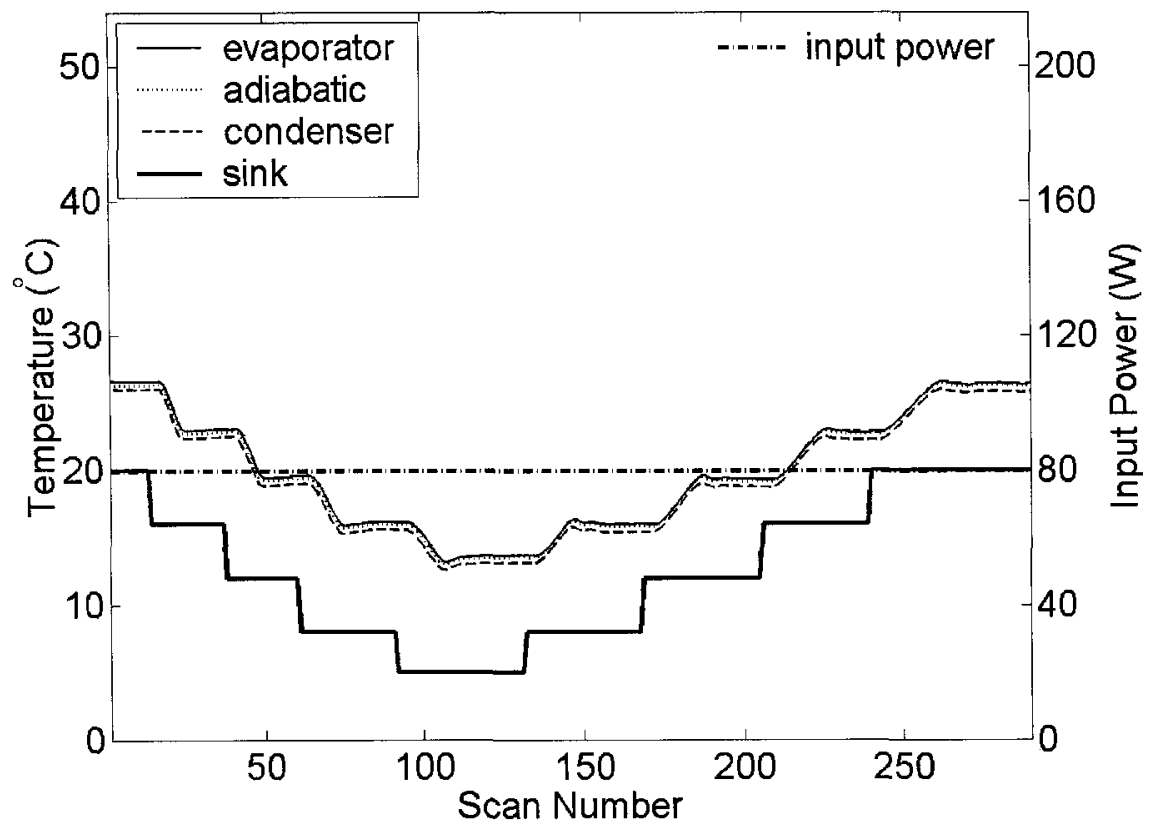

Figure 2.19: AGHP, Average Temperature, Source $80 \mathrm{~W}$

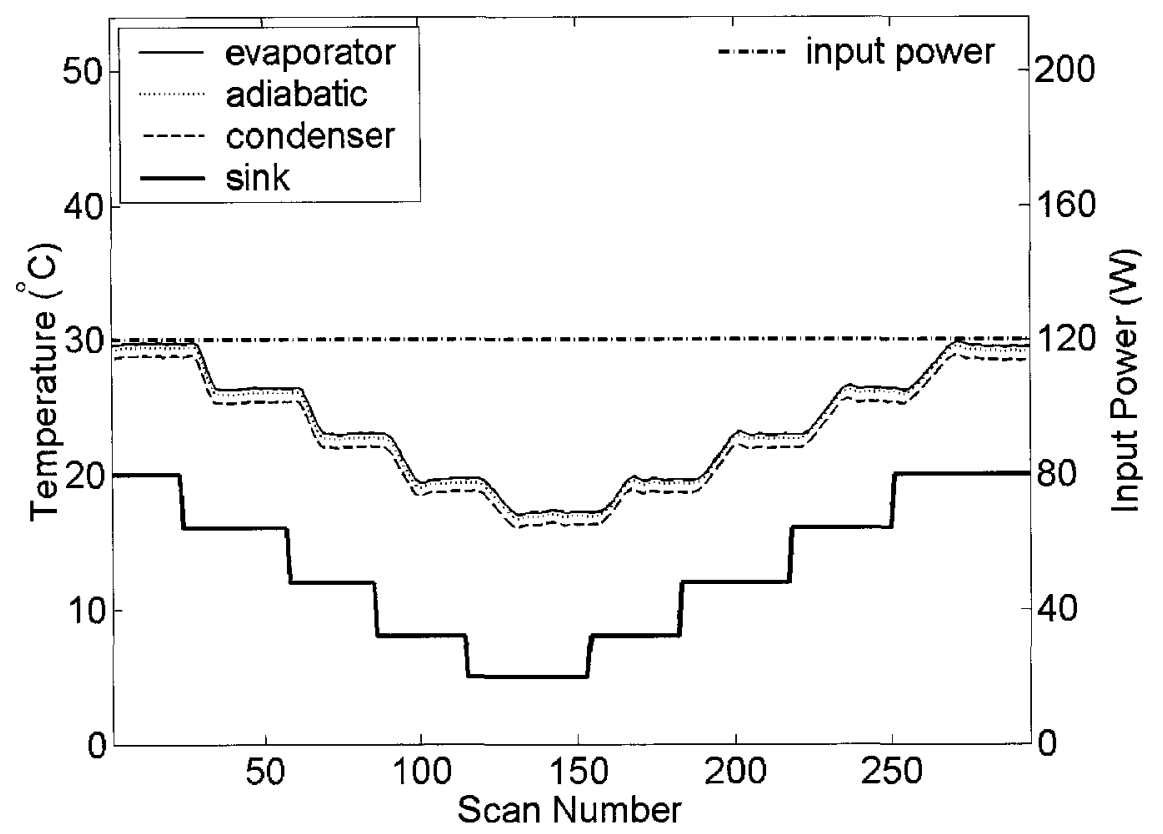

Figure 2.20: AGHP, Average Temperature, Source $120 \mathrm{~W}$ 


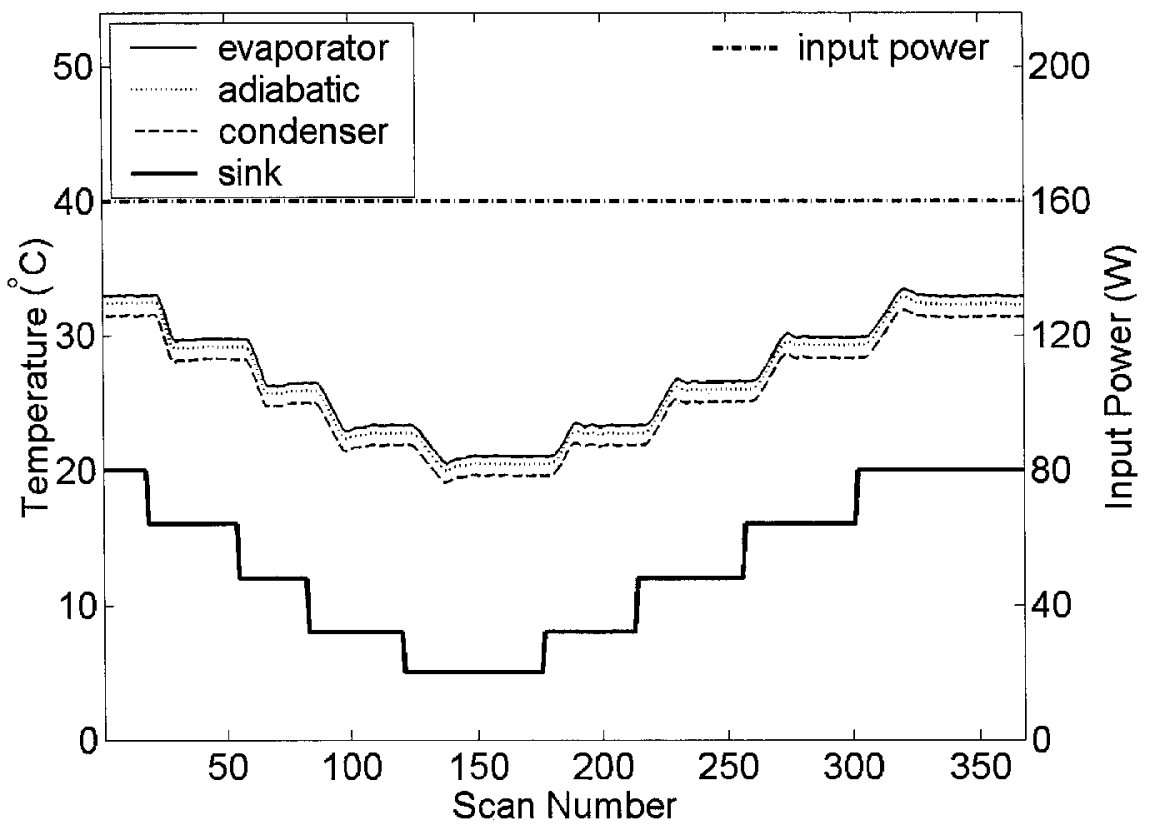

Figure 2.21: AGHP, Average Temperature, Source $160 \mathrm{~W}$

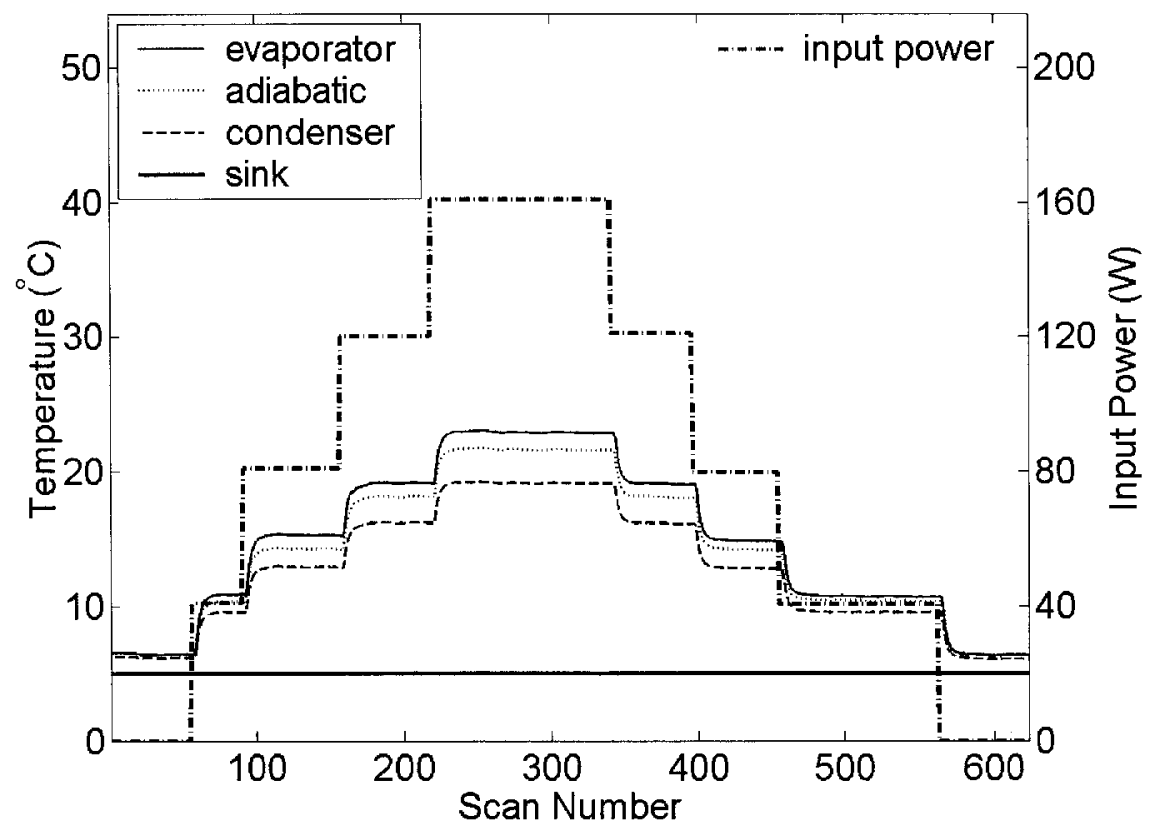

Figure 2.22: AHP, Average Temperature, Sink $5{ }^{\circ} \mathrm{C}$ 


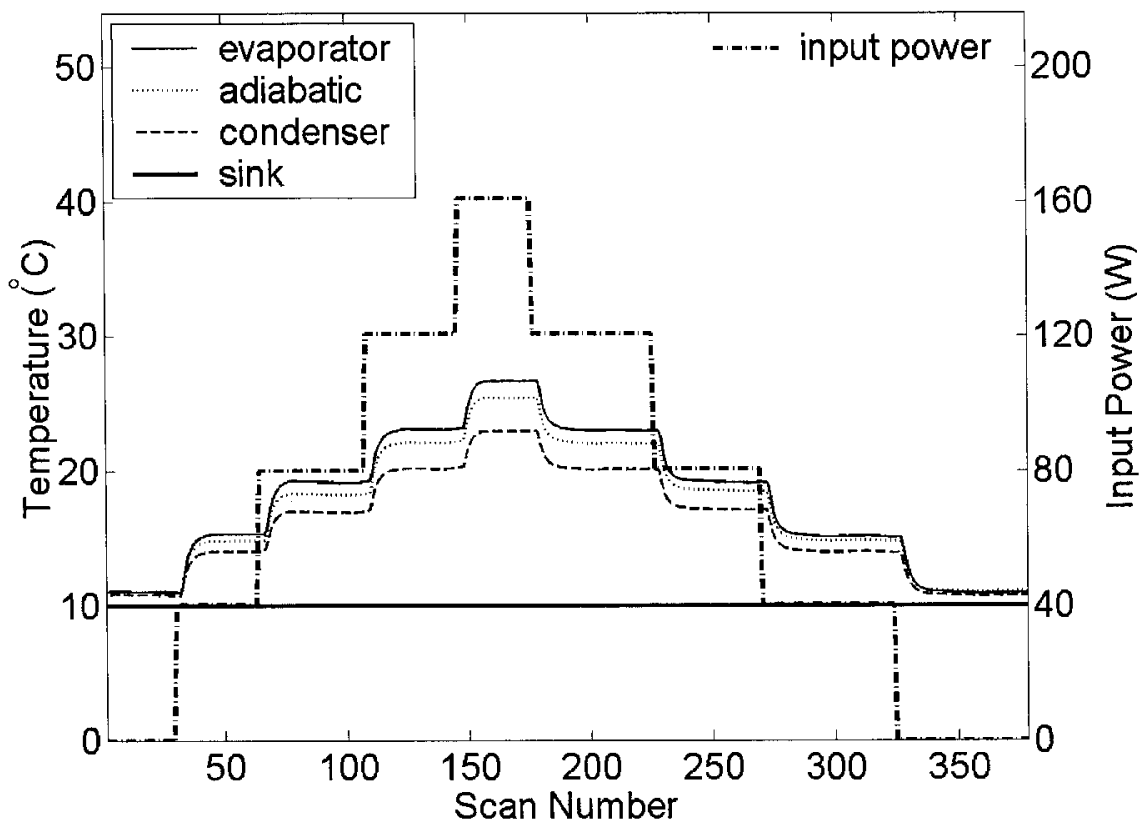

Figure 2.23: AHP, Average Temperature, Sink $10^{\circ} \mathrm{C}$

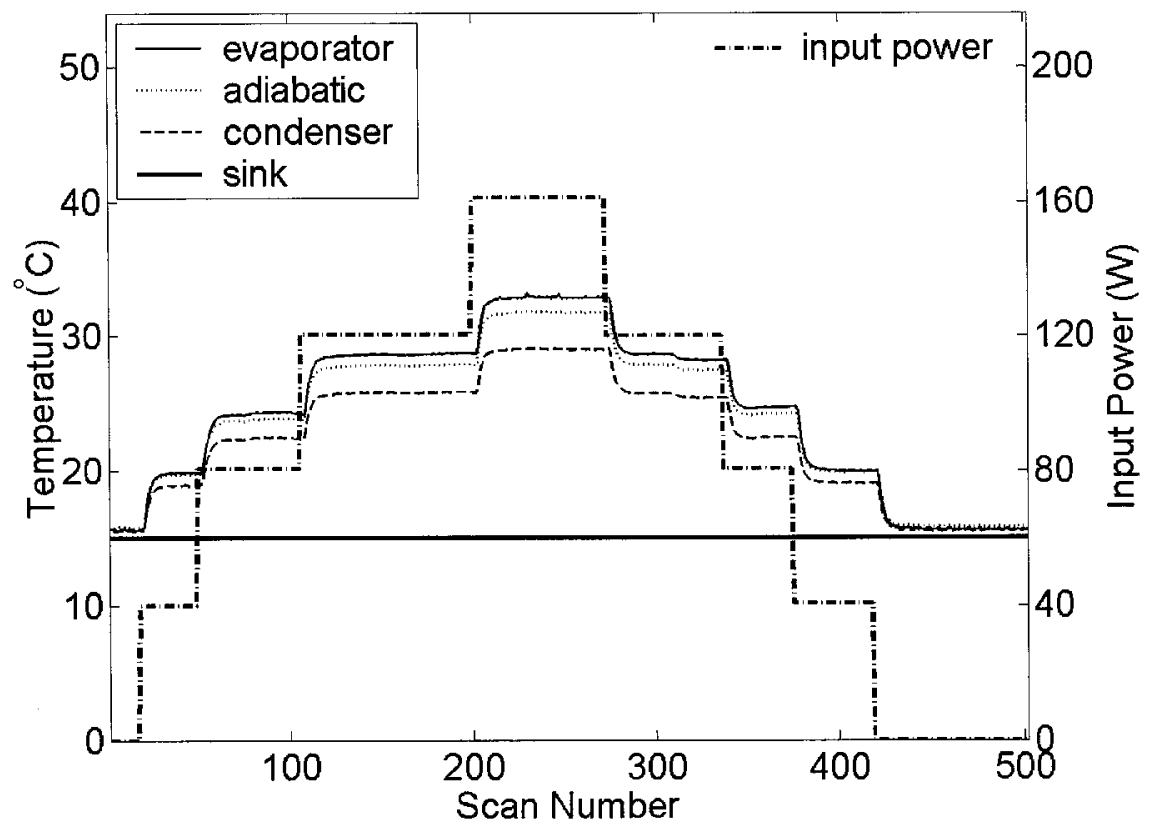

Figure 2.24: AHP, Average Temperature, Sink $15^{\circ} \mathrm{C}$ 


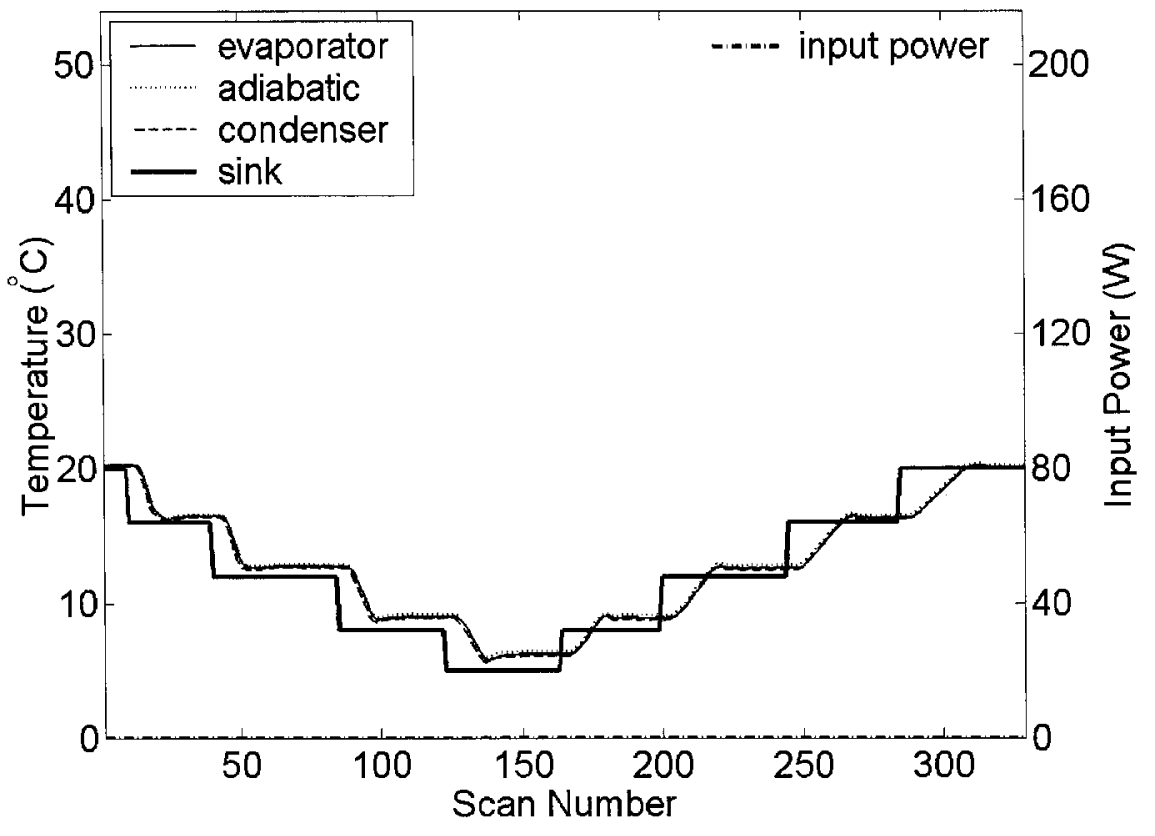

Figure 2.25: AHP, Average Temperature, Source $0 \mathrm{~W}$

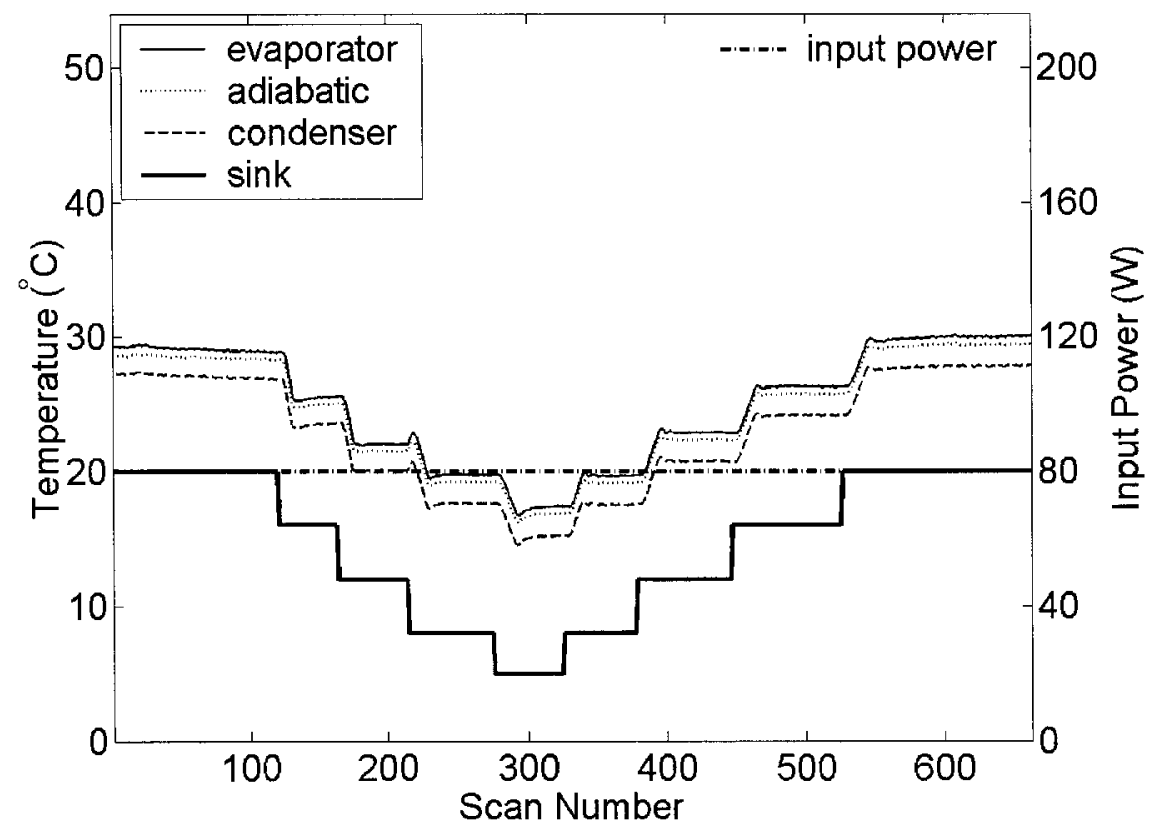

Figure 2.26: AHP, Average Temperature, Source $80 \mathrm{~W}$ 


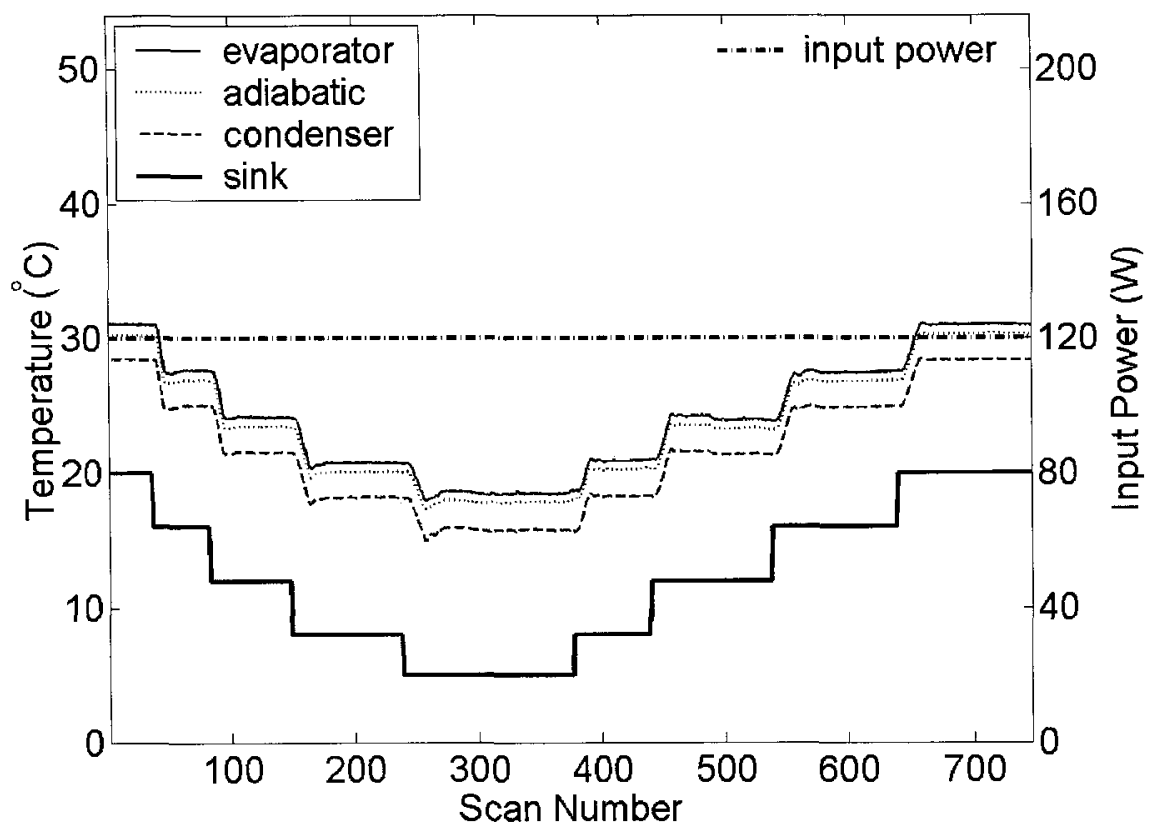

Figure 2.27: AHP, Average Temperature, Source $120 \mathrm{~W}$

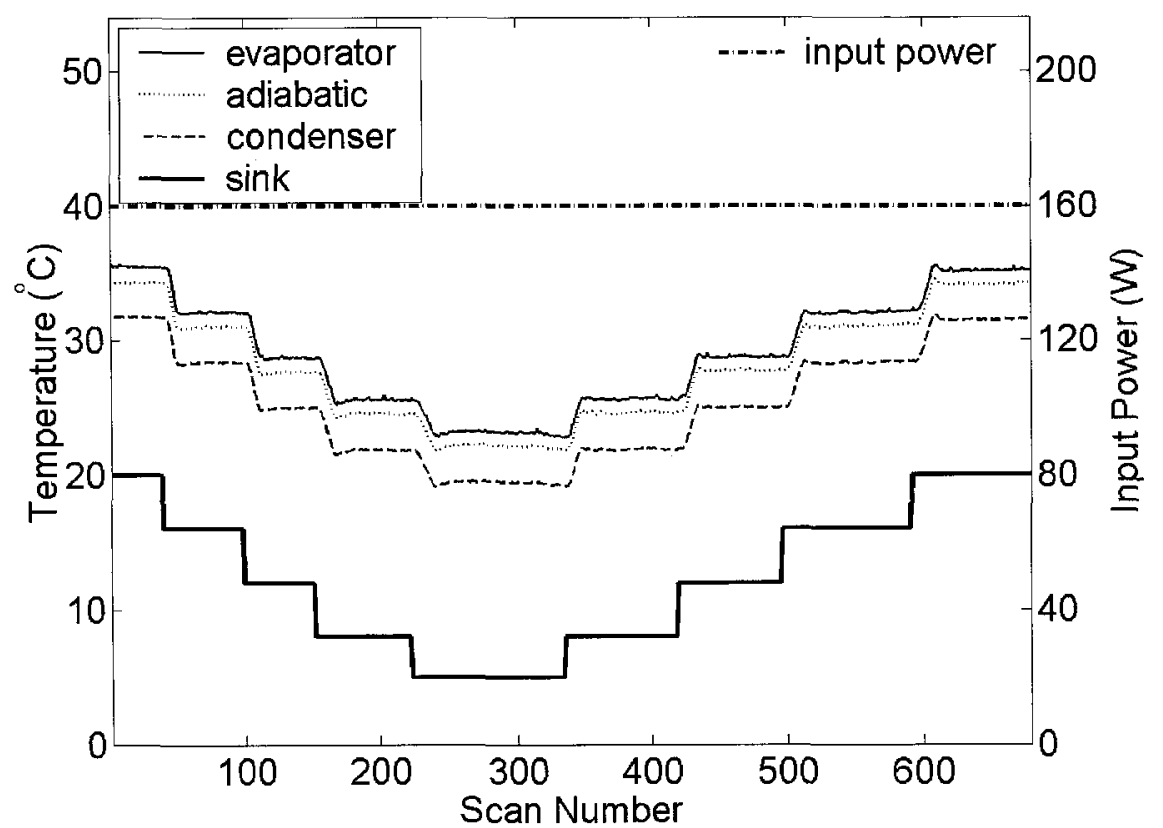

Figure 2.28: AHP, Average Temperature, Source $160 \mathrm{~W}$ 


\subsubsection{Spatial Temperature Variation}

In the graphical representation of the variation in temperature with position, the position station numbers along the abscissa increase marching from the evaporator to the condenser. The temperature is taken at steady-state two scan cycles before heat input or sink temperature change.

For the AGHP, Figures 2.29, 2.30, 2.31 show the spatial temperature variation with constant sink temperature and variable heat input, and Figures 2.32, 2.33, 2.34, 2.35, 2.36 show the spatial temperature variation with constant heat input and variable sink temperature.

For the AHP, Figures 2.37, 2.38, 2.39 show the spatial temperature variation with constant sink temperature and variable heat input, and Figures 2.40, 2.41, 2.42, 2.43 show the spatial temperature variation with constant heat input and variable sink temperature.

Given that heat is primarily transferred through a phase change, a small temperature difference is expected between the evaporator and condenser. This is observed in the set of figures mentioned above. Comparing the above figures, one can conclude the AGHP performs more isothermally than the AHP. Also, the change in temperature with respect to position is more pronounced at positions where the adiabatic section makes contact with either the evaporator or the condenser. This change is also larger with the AHP compared to the AGHP.

Naturally, a higher heat input led to higher position temperatures. A higher heat input also led to a greater temperature change between the evaporator and the condenser. This total drop was also greater with the AHP compared to the AGHP. 
An increase in temperature travelling from stations 1-5 is seen in Figures 2.37 and 2.40. This is thought to be due to cooling from the liquid arriving from the condenser.

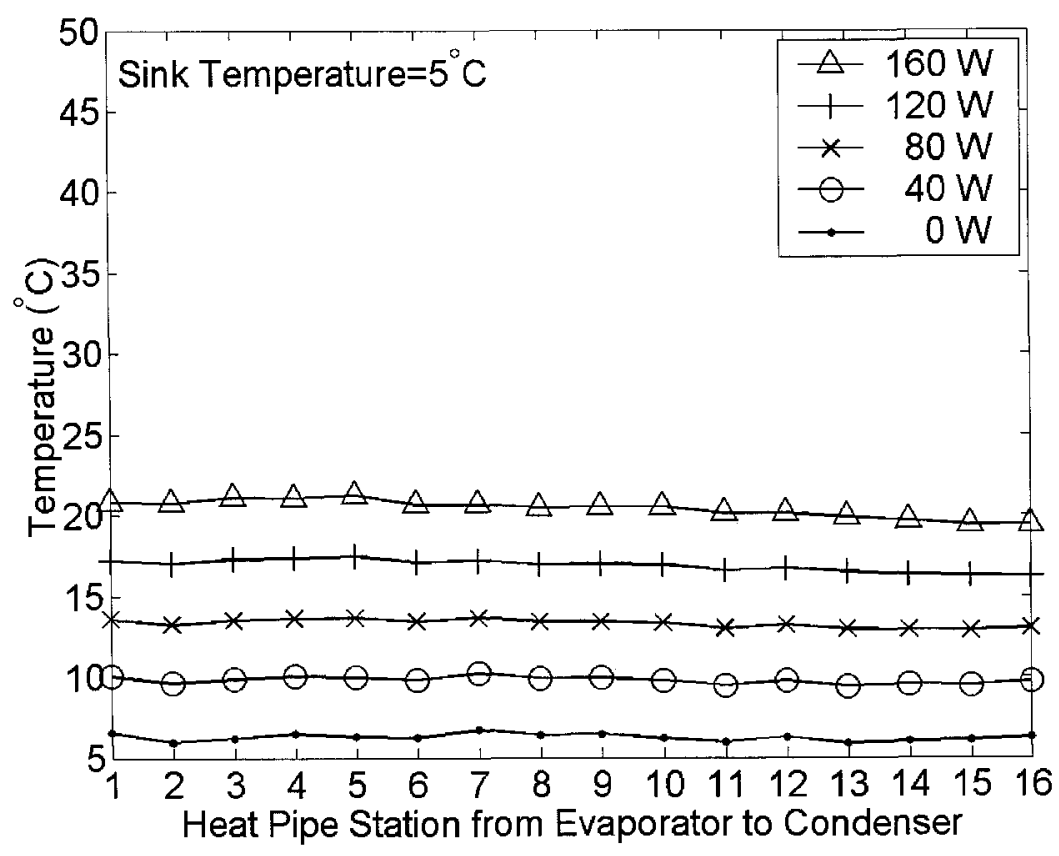

Figure 2.29: AGHP, Station Temperature, Sink $5{ }^{\circ} \mathrm{C}$ 


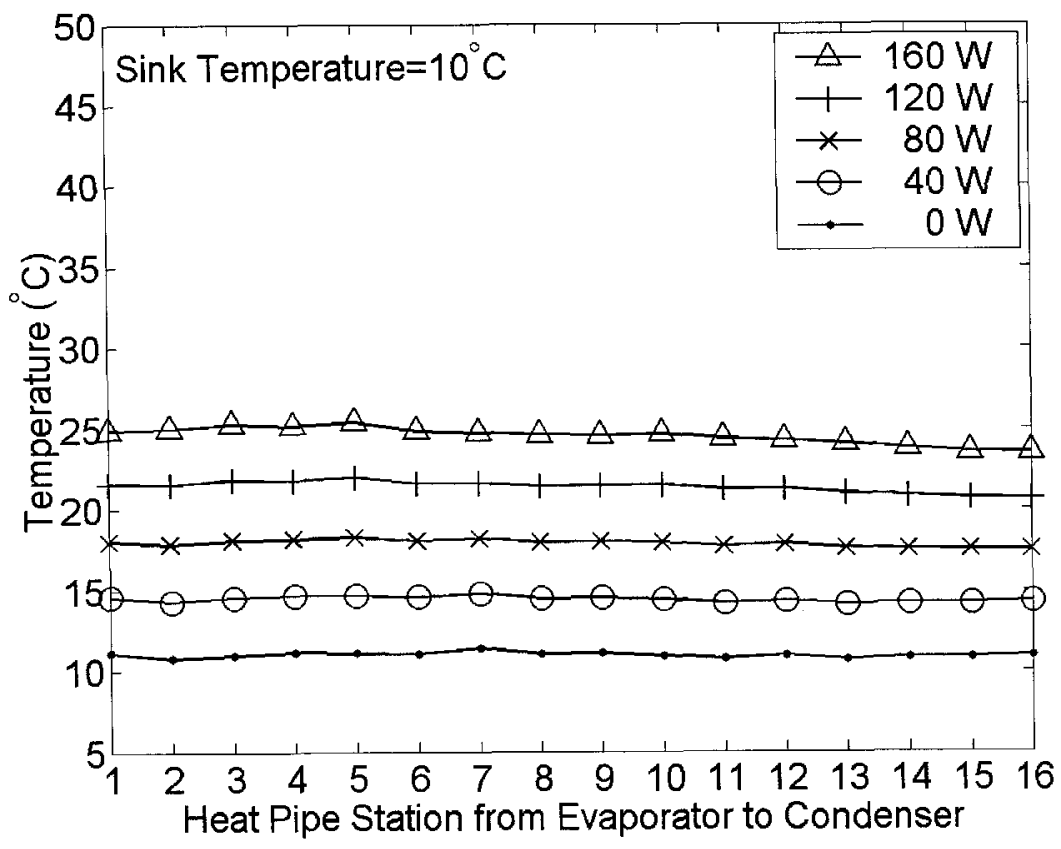

Figure 2.30: AGHP, Station Temperature, Sink $10^{\circ} \mathrm{C}$

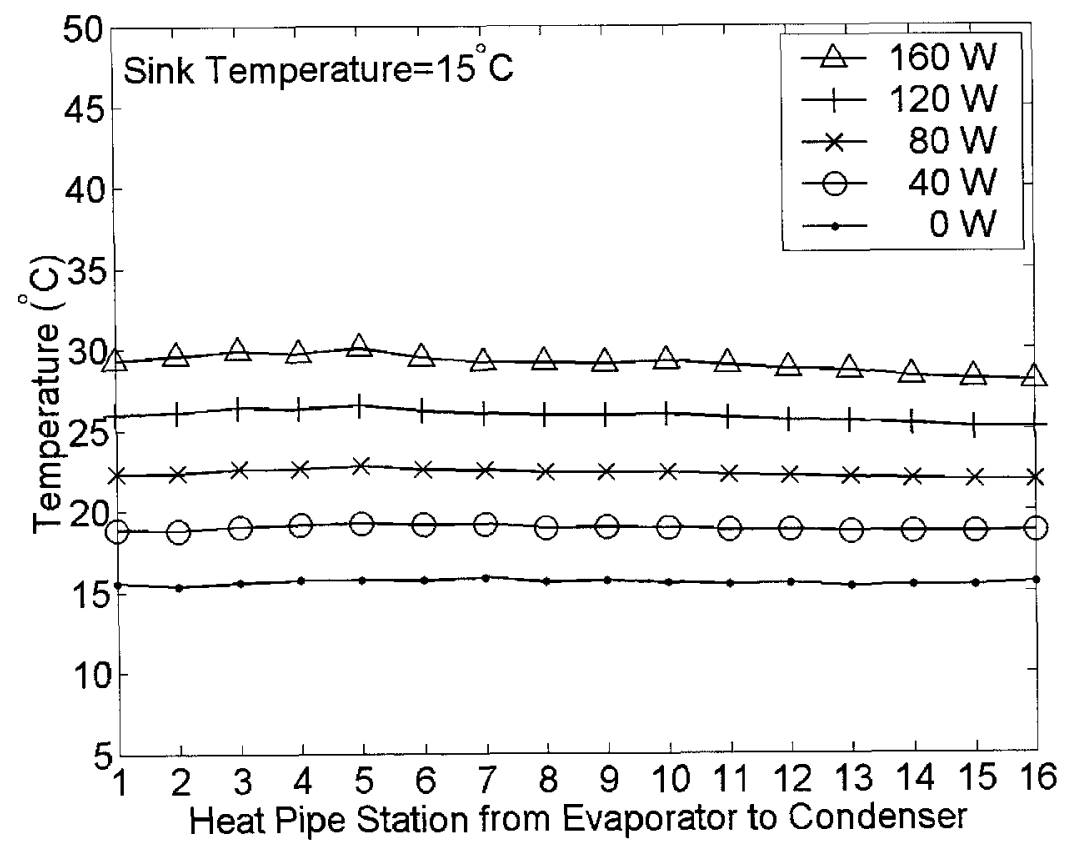

Figure 2.31: AGHP, Station Temperature, Sink $15^{\circ} \mathrm{C}$ 


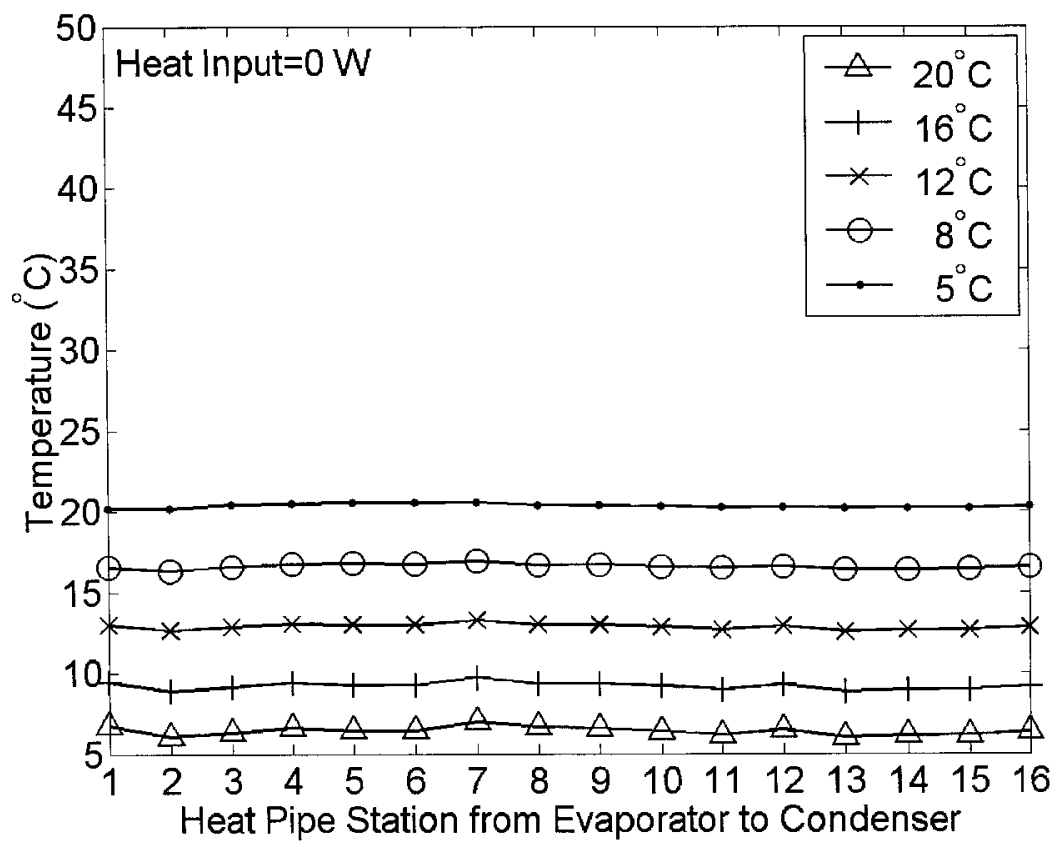

Figure 2.32: AGHP, Station Temperature, Source $0 \mathrm{~W}$

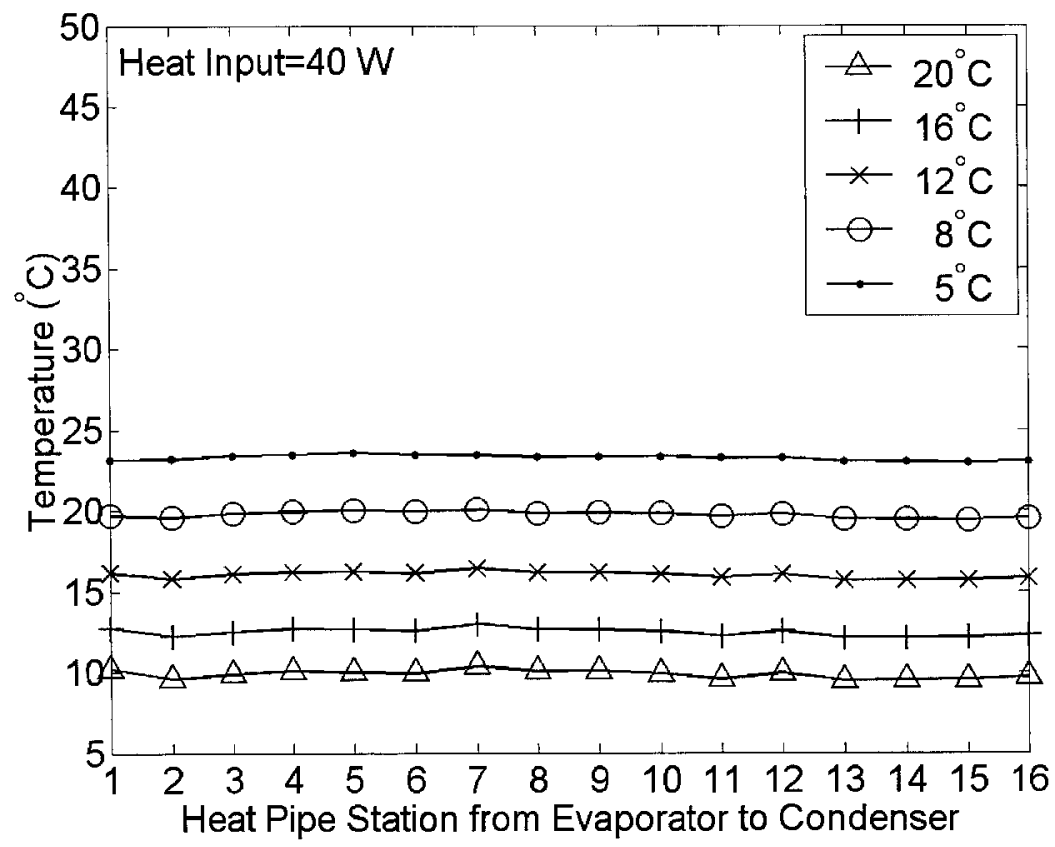

Figure 2.33: AGHP, Station Temperature, Source $40 \mathrm{~W}$ 


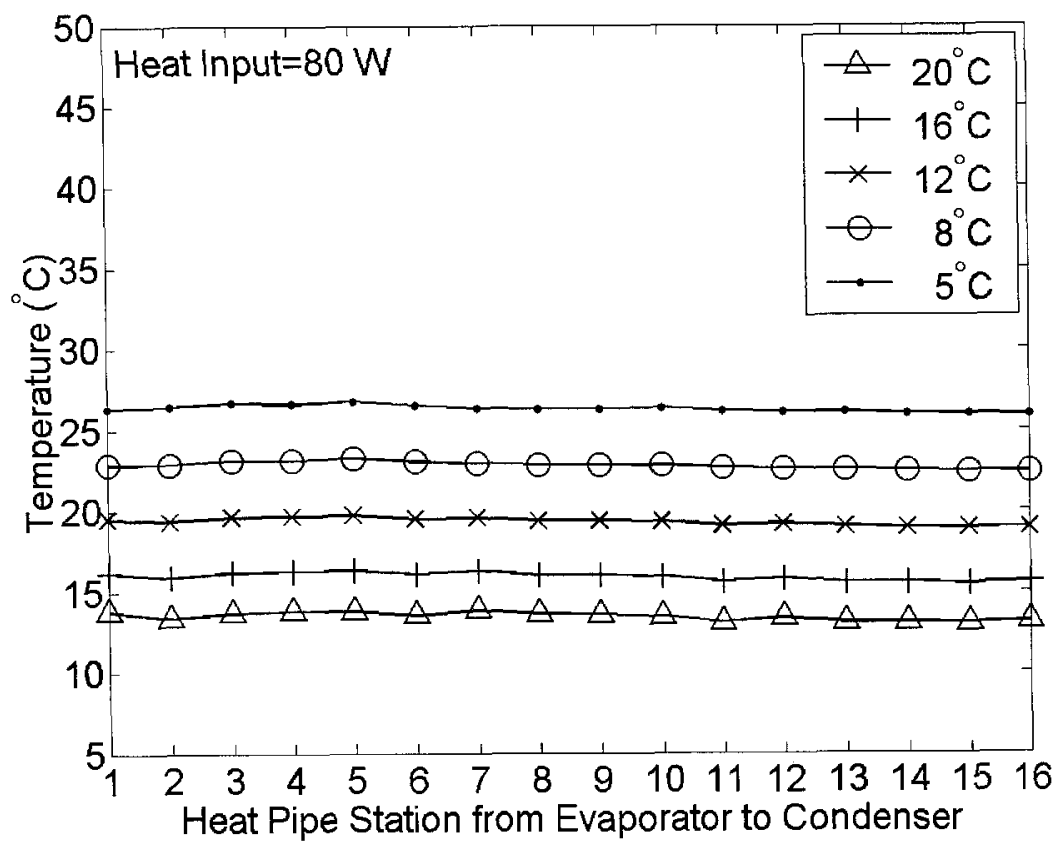

Figure 2.34: AGHP, Station Temperature, Source $80 \mathrm{~W}$

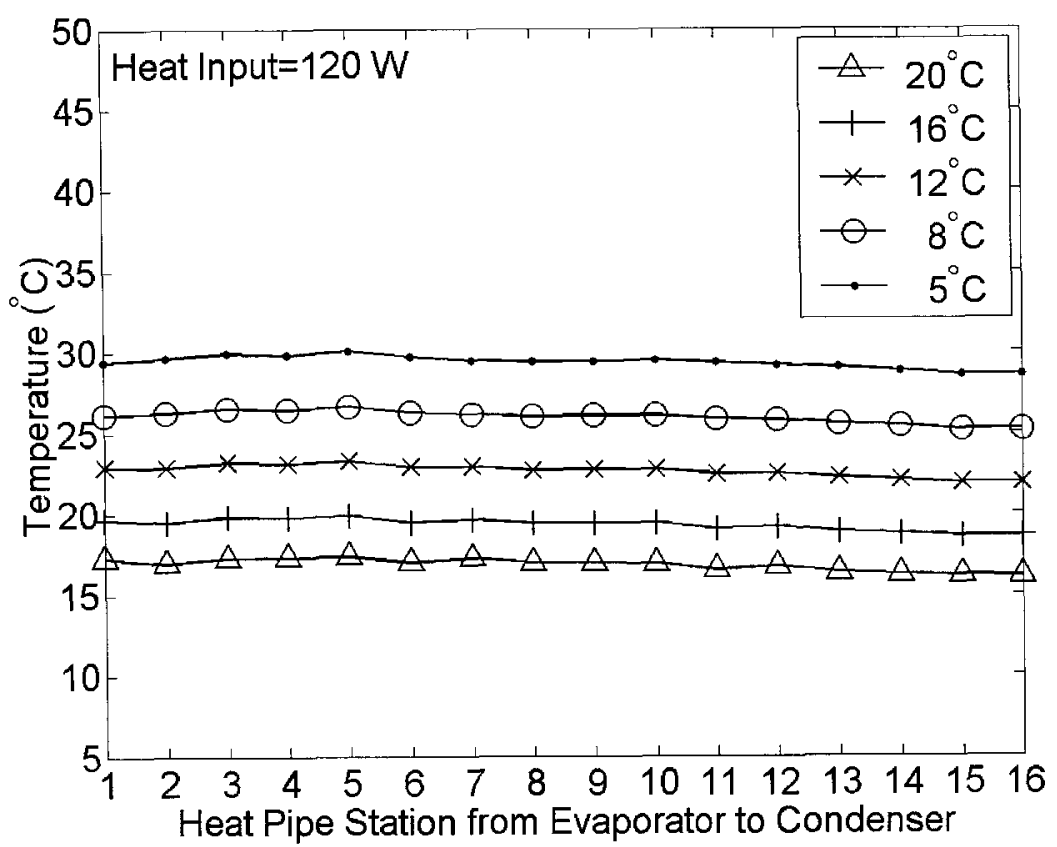

Figure 2.35: AGHP, Station Temperature, Source $120 \mathrm{~W}$ 


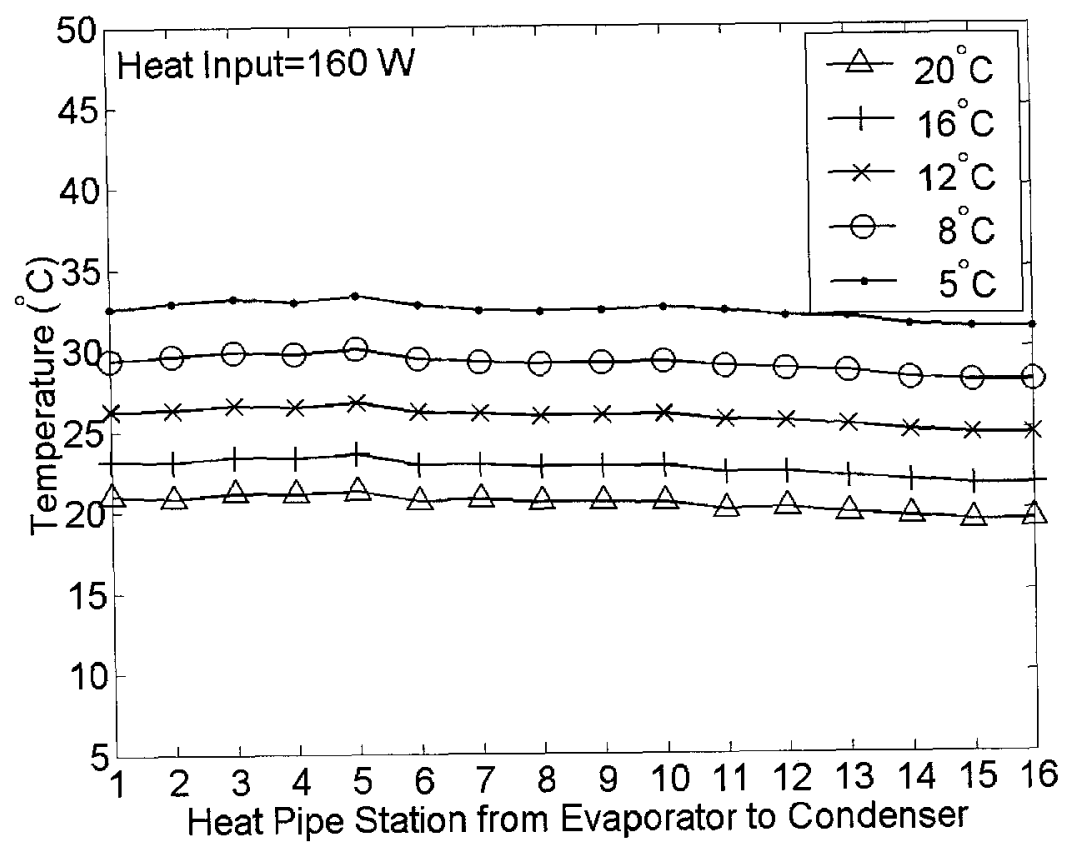

Figure 2.36: AGHP, Station Temperature, Source $160 \mathrm{~W}$

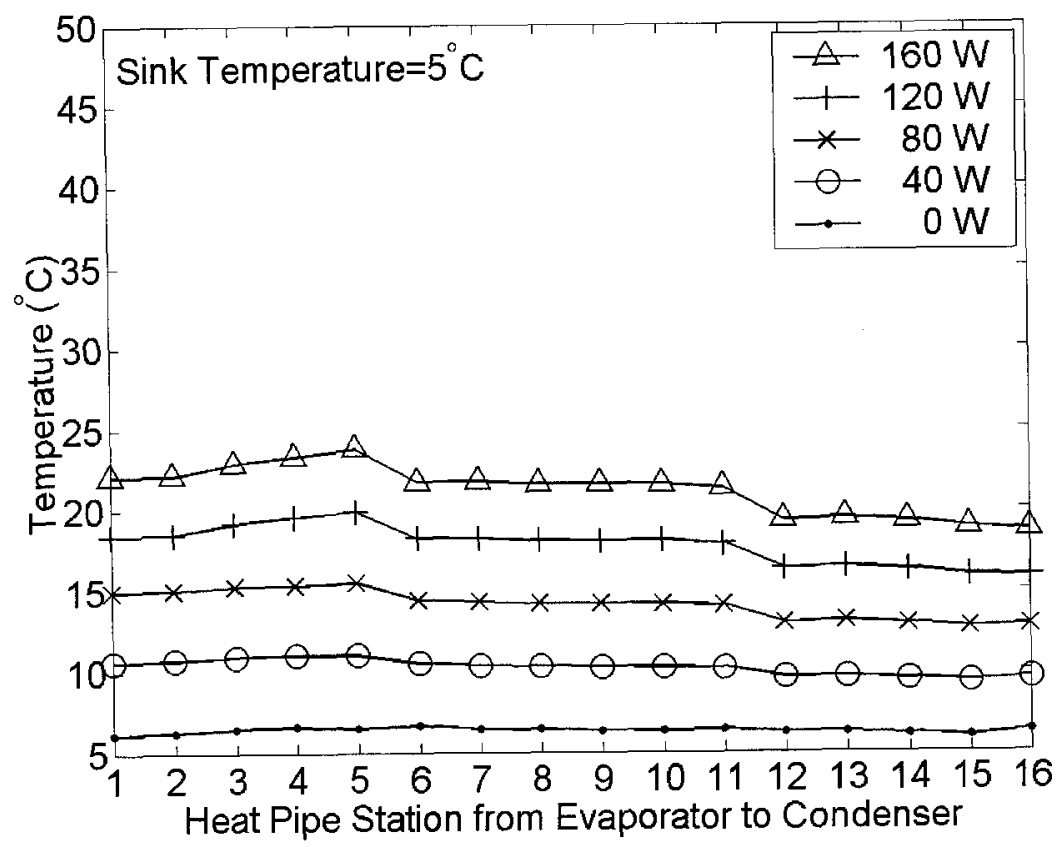

Figure 2.37: AHP, Station Temperature, Sink $5{ }^{\circ} \mathrm{C}$ 


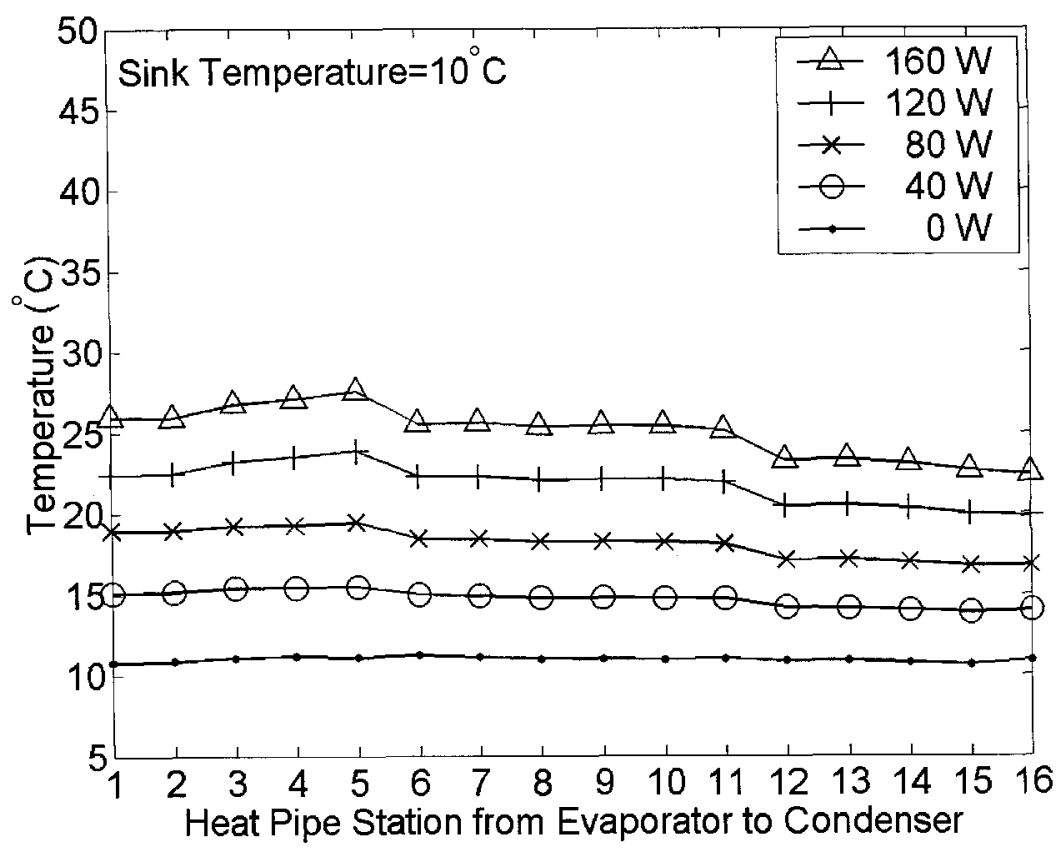

Figure 2.38: AHP, Station Temperature, Sink $10^{\circ} \mathrm{C}$

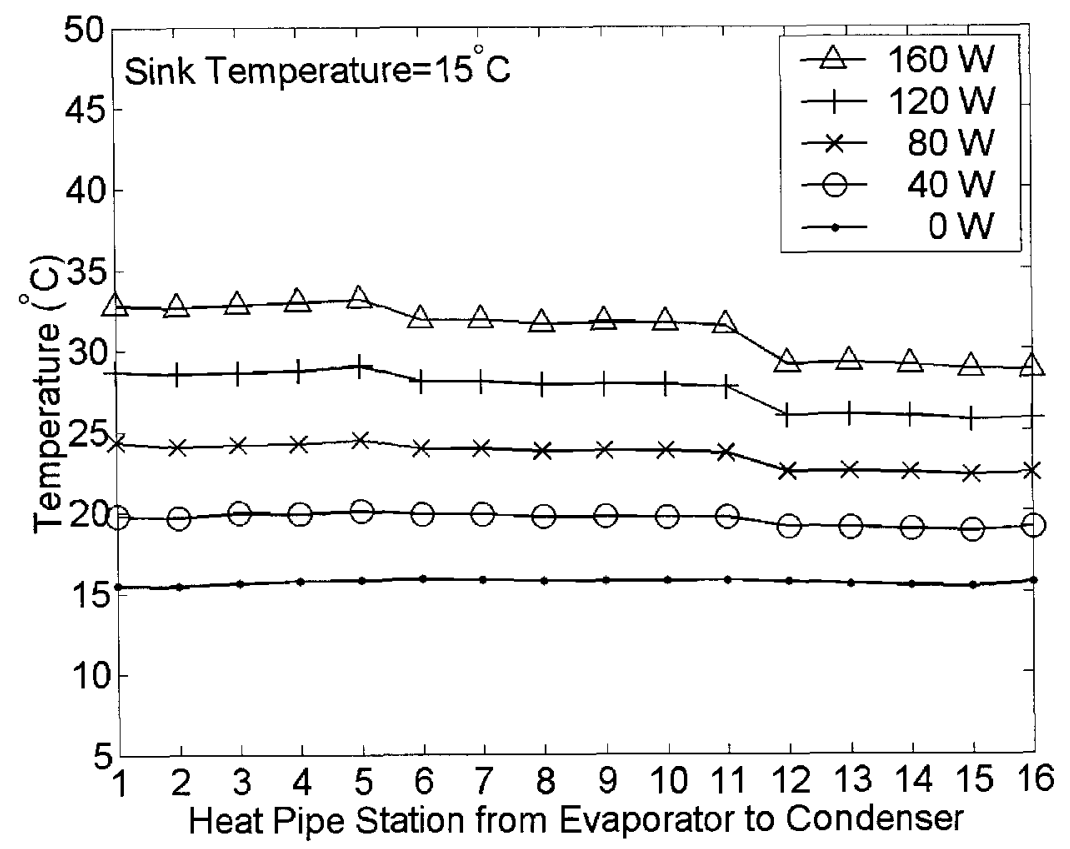

Figure 2.39: AHP, Station Temperature, Sink $15^{\circ} \mathrm{C}$ 


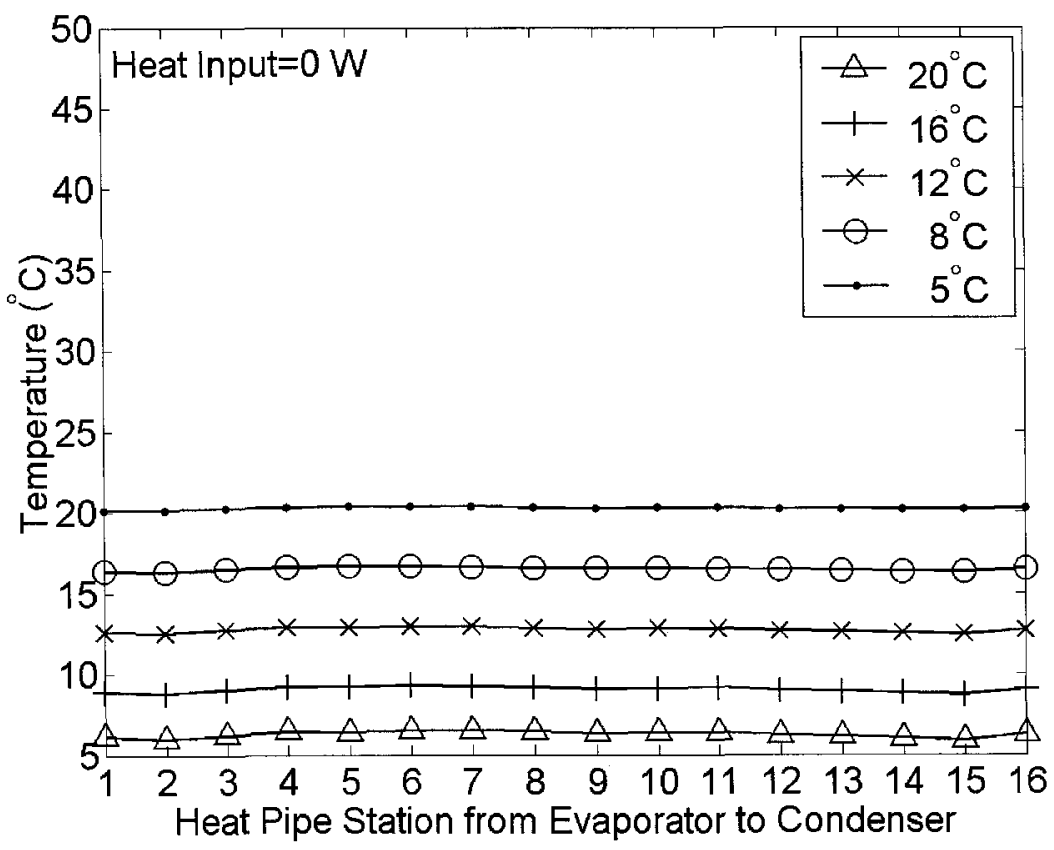

Figure 2.40: AHP, Station Temperature, Source $0 \mathrm{~W}$

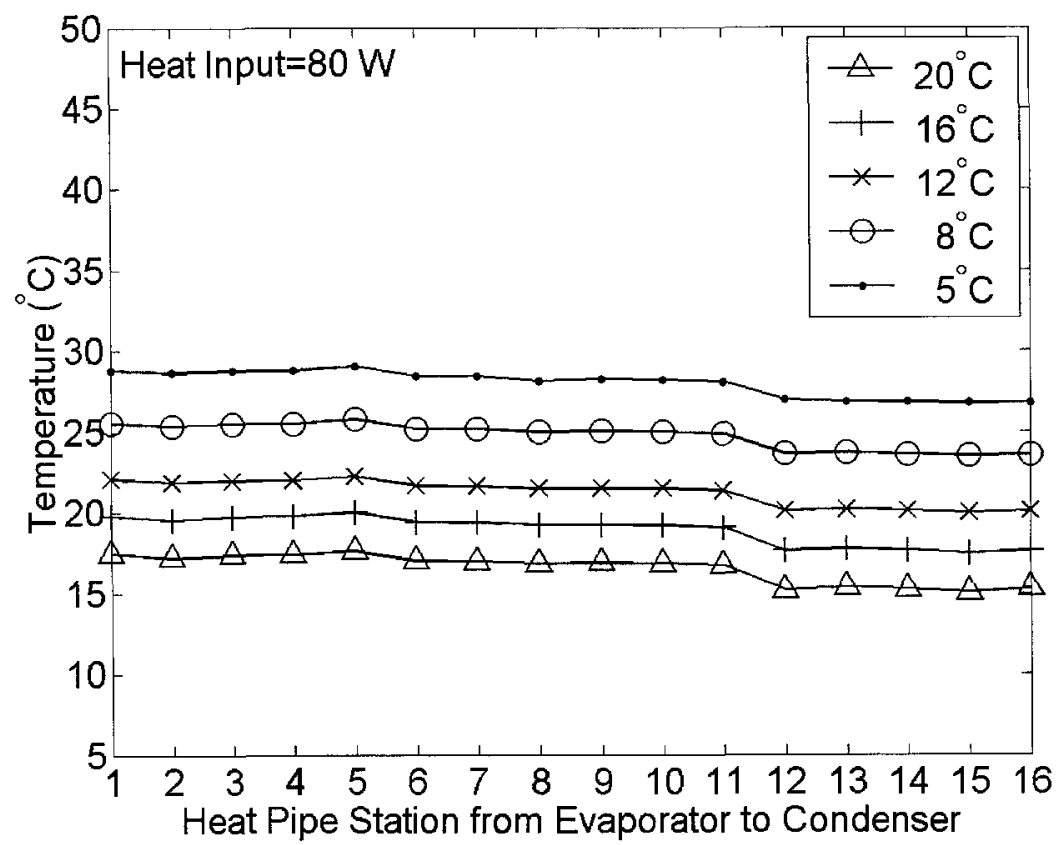

Figure 2.41: AHP, Station Temperature, Source $80 \mathrm{~W}$ 


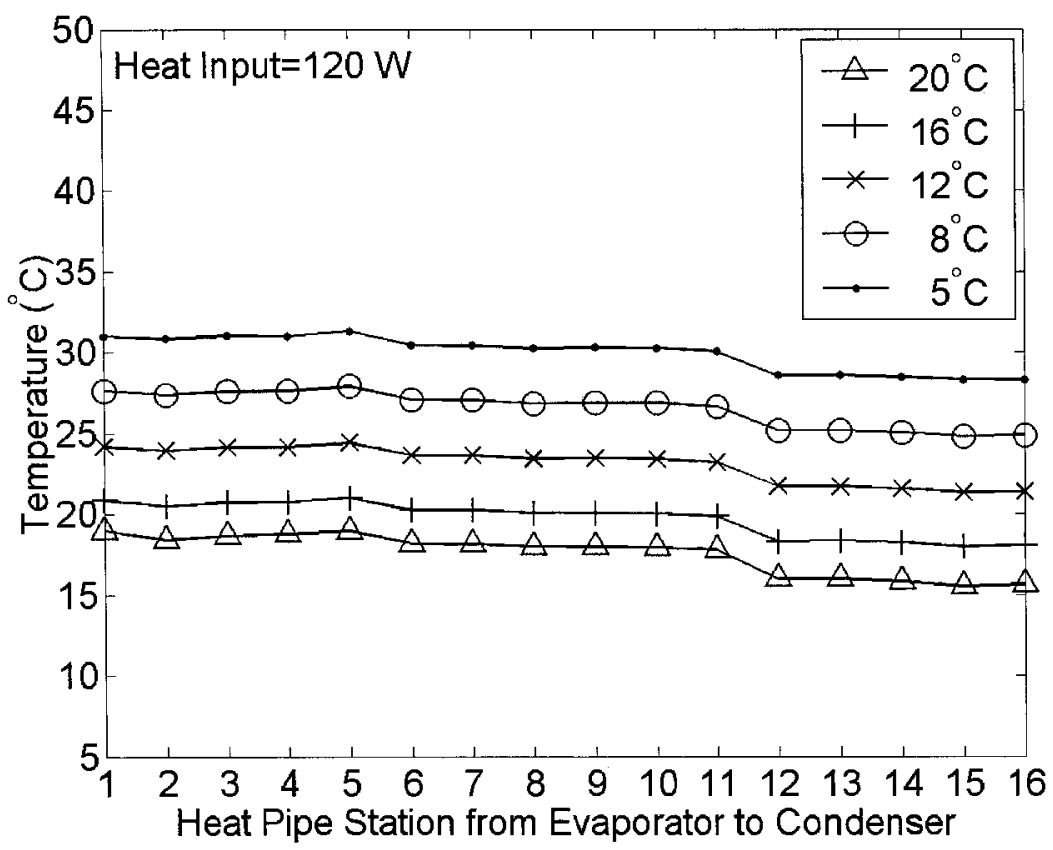

Figure 2.42: AHP, Station Temperature, Source $120 \mathrm{~W}$

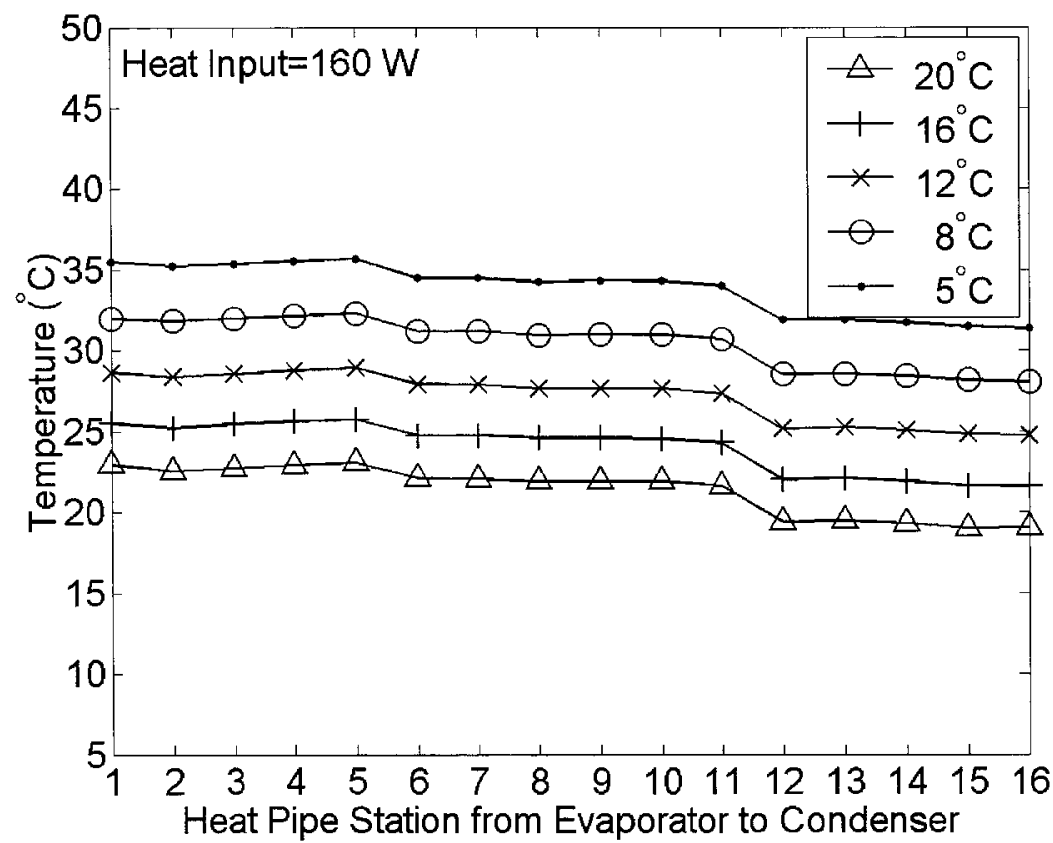

Figure 2.43: AHP, Station Temperature, Source $160 \mathrm{~W}$ 


\subsubsection{Effective Thermal Conductivity}

A well established parameter for comparing heat transfer abilities of different devices is effective thermal conductivity $\lambda_{\text {eff }}$ isolated from Fourier's law seen in Eq.(2.4), where $A$ is the heat pipe cross-sectional area, $\Delta T$ is the temperature difference between thermocouples 7 and 20 on the evaporator and condenser with reference to Figure 2.12. The average evaporator and condenser temperatures could also be used to define $\Delta T$.

$$
\lambda_{\mathrm{eff}}=\frac{Q L_{\mathrm{eff}}}{A \Delta T}
$$

The heat transfer $Q$ is taken as the power input under the assumption that there is no heat loss. The effective length, $L_{\text {eff }}$ of the heat pipe is not equal to the actual length since the axial heat transfer is not uniform along the heat pipe length. Assuming axial heat transfer is zero at the heat pipe ends and changes linearly and symmetrically along the evaporator and condenser, one can obtain the effective length in Eq.(2.5), Ref.[15].

$$
L_{\mathrm{eff}}=0.5 L_{\mathrm{ev}}+L_{\mathrm{ad}}+0.5 L_{\mathrm{co}}
$$

AGHP and AHP effective thermal conductivity as functions of heat input for different sink temperatures can be seen in Figure 2.44 and Figure 2.45, respectively. Dotted lines were used to connect points only for clarity and are not meant to suggest a linear relationship. The data used was from the test portion with increasing heat input. Thermal conductivity for the latter part of the test with decreasing heat input was not included because hysteresis was not observed.

One can see from Figure 2.44 and Figure 2.45 that effective thermal conductivity for the most part decreases with increased heat input. The AHP conductivity 


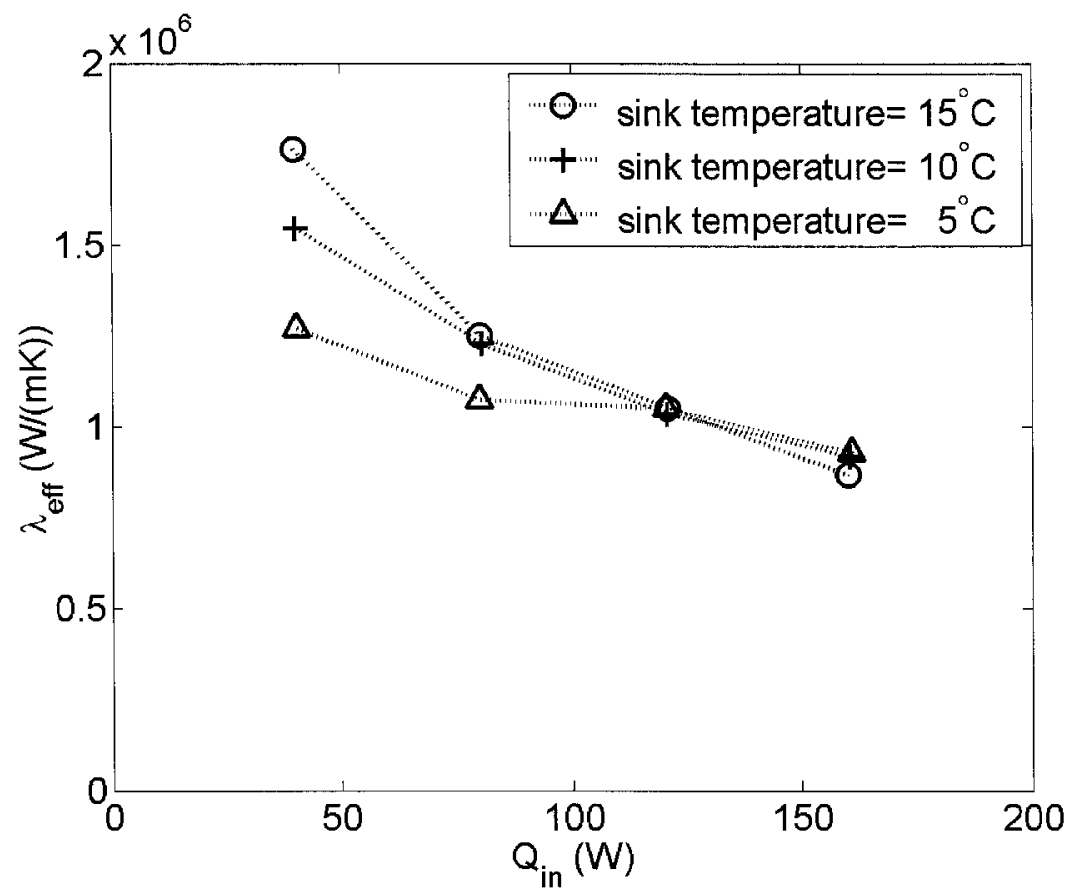

Figure 2.44: AGHP Effective Thermal Conductivity

decreases drastically from $40 \mathrm{~W}$ to $80 \mathrm{~W}$. The AGHP conductivity does drop with increased heat input, but the conductivity decreases less and is greater than the AHP. One reason for which the effective thermal conductivity of the AGHP is higher than for the AHP is due to the higher conductivity of the AGHP body made of aluminum compared to the stainless steel AHP body.

Higher sink temperatures give higher conductivity for the AGHP at low heat input. The reverse occurs at higher heat input as can be seen in Figure 2.44. This trend is not so much observed with the AHP as its performance seems nearly independent of sink temperature based on the overlapping curves in Figure 2.45. 


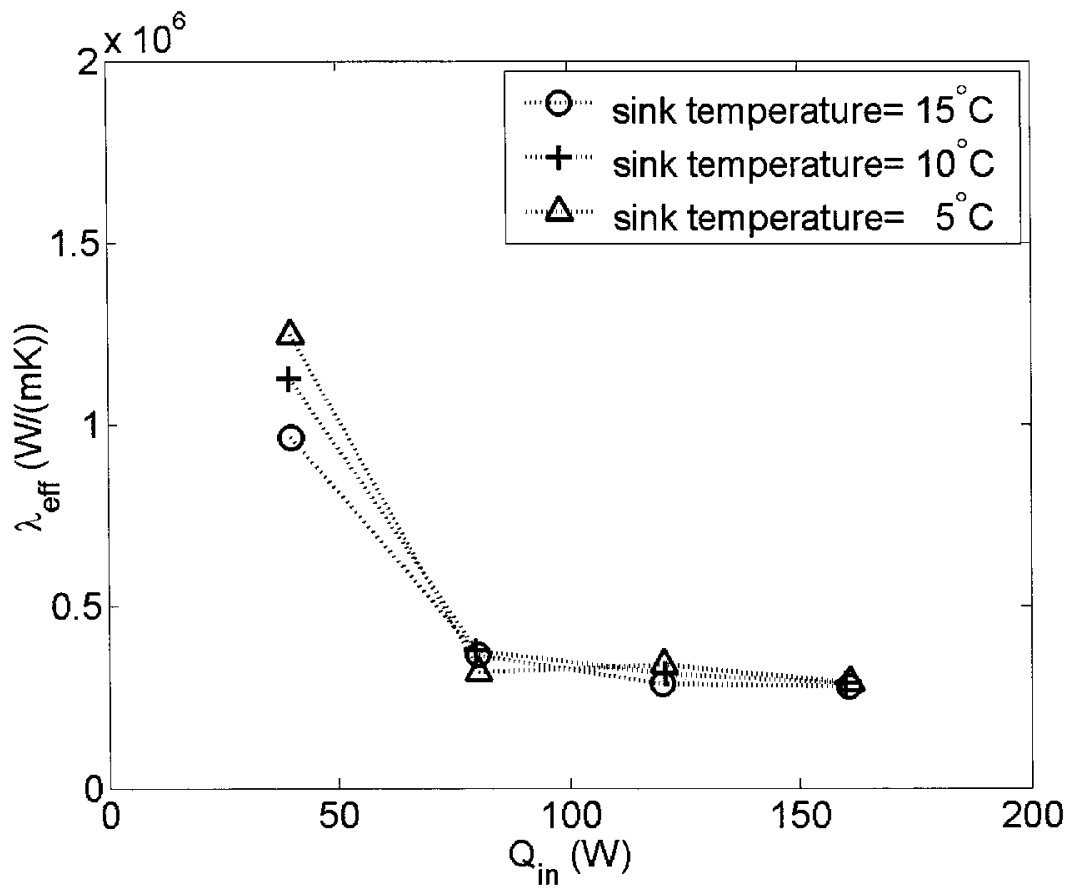

Figure 2.45: AHP Effective Thermal Conductivity

\subsubsection{Heat Balance}

Performing a heat balance between the evaporator and the condenser is of importance to justify the assumption of perfect insulation.

\section{Heat Addition}

The applied load is assumed to be purely resistive. This makes the phase angle $\Upsilon=0$ and the input power becomes

$$
\begin{aligned}
Q_{i n} & =I_{\mathrm{RMS}} V_{\mathrm{RMS}} \cos (\Upsilon) \\
& =I_{\mathrm{RMS}} V_{\mathrm{RMS}}
\end{aligned}
$$

The calculation of heat output is more involved. 


\section{Heat Extraction}

It is of interest to analyze the heat output by the condenser to the heat exchanger. Calculating the condenser heat output is done by applying the first law of thermodynamics across the heat exchanger from which we get:

$$
Q_{\text {out }}=\rho_{c} \dot{V} c_{p_{\text {mix }}}\left(T_{\text {out }}-T_{\text {in }}\right)
$$

where $\rho_{c}$ is the coolant density, $\dot{V}$ is the volumetric coolant flow rate, $c_{p_{\text {mix }}}$ is the coolant specific heat, and $T_{\text {out }}-T_{\text {in }}$ is the temperature difference across the heat exchanger. One can obtain the coolant specific heat by combining the specific heats of its constituents. In this case, the coolant is $70 \%$ water and $30 \%$ laboratory grade ethylene glycol, by volume. Given the specific heat and specific volume of each component, The mixture specific heat based on a weighted mass average is shown in Eq.(2.8):

$$
c_{p_{m i x}}=\frac{\left(v \rho c_{p}\right)_{\mathrm{eg}}+\left(v \rho c_{p}\right)_{\mathrm{w}}}{(v \rho)_{\mathrm{eg}}+(v \rho)_{\mathrm{w}}}
$$

Specific heat as a function of temperature in Kelvin for ethylene glycol and water is obtained from Ref.[22] with polynomial coefficients shown in Tab.(2.1)

$$
\begin{gathered}
c_{p_{\text {eg }}}=\alpha\left(a_{0}+a_{1} T-a_{2} T^{2}+a_{3} T^{3}\right)\left(\frac{\mathrm{J}}{\mathrm{kgK}}\right) \\
c_{p_{\mathrm{w}}}=\alpha\left(a_{0}-a_{1} T+a_{2} T^{2}-a_{3} T^{3}\right)\left(\frac{\mathrm{J}}{\mathrm{kgK}}\right)
\end{gathered}
$$


Table 2.1: Specific Heat Coefficients, Ref.[22]

\begin{tabular}{|c|r|r|}
\hline Coefficient & $c_{p e g}$ & $c_{w}$ \\
\hline$\alpha$ & $4.184 \mathrm{E} 6$ & $4.184 \mathrm{E} 6$ \\
$a_{0}$ & 0.016884 & 2.13974 \\
$a_{1}$ & $3.35083 \mathrm{E}-3$ & $9.68137 \mathrm{E}-3$ \\
$a_{2}$ & $7.224 \mathrm{E}-6$ & $2.68536 \mathrm{E}-5$ \\
$a_{3}$ & $7.61748 \mathrm{E}-9$ & $2.42139 \mathrm{E}-8$ \\
\hline
\end{tabular}

\section{Uncertainty Analysis}

The input and output heat calculations based on measurements both have a degree of uncertainty. The general formula for error propagation given a multivariate function; $z=z(x, y, \ldots)$ is given by Eq.(2.11), Ref.[23].

$$
\delta_{z}=\sqrt{\left(\frac{\partial z}{\partial x} \delta_{x}\right)^{2}+\left(\frac{\partial z}{\partial y} \delta_{y}\right)^{2}+\ldots}
$$

The uncertainty for the heat into the evaporator from Eq.(2.6) is seen in Eq.(2.12).

$$
\delta_{Q_{i n}}=\sqrt{\left(V_{\mathrm{RMS}} \delta_{I_{\mathrm{RMS}}}\right)^{2}+\left(I_{\mathrm{RMS}} \delta_{V_{\mathrm{RMS}}}\right)^{2}}
$$

A linear relation between current and voltage (Ohm's law) was experimentally found by correlating them inside the test domain so evaluation of the error could be performed. For the heat output uncertainty, the temperature difference across the heat exchanger was calculated with Eq.(2.14) before proceeding to the calculation the output heat uncertainty in Eq.(2.15).

$$
\Delta T=T_{\text {out }}-T_{\text {in }}
$$




$$
\begin{gathered}
\delta_{\Delta T}=\sqrt{\left(\delta_{T_{\text {out }}}\right)^{2}+\left(\delta_{T_{\text {in }}}\right)^{2}} \\
\delta_{Q_{\text {out }}}=\rho \sqrt{\left(c_{p} \Delta T \delta_{\dot{V}}\right)^{2}+\left(\dot{V} \Delta T \delta_{c_{p}}\right)^{2}+\left(\dot{V} c_{p} \delta_{\Delta_{T}}\right)^{2}}
\end{gathered}
$$

Graphical representation of Eq.(2.12) given the values in Tab.(2.2) in shown in Fig-

Table 2.2: Property Uncertainty

\begin{tabular}{|c|c|}
\hline Property & Uncertainty \\
\hline$V_{R M S}$ & $0.16 \mathrm{~V}$ \\
$I_{R M S}$ & $0.3 \mathrm{~A}$ \\
$\dot{V}$ & $0.0041 \frac{\mathrm{L}}{\mathrm{s}}$ \\
$c_{p}$ & $100 \frac{\mathrm{J}}{\mathrm{kgK}}$ \\
$T_{\text {in }}, T_{\text {out }}$ & $1^{\circ} \mathrm{C}$ \\
\hline
\end{tabular}

ure 2.46. The uncertainty associated with the heat output from Eq.(2.15) was found to be orders of magnitude larger than the uncertainty associated heat input measurement. This is due to the fact that heat exchanger input and output temperatures are nearly equal for small heat transfer.

The heat transfer range is limited by the operational restrictions placed on the heat pipe and its subsystems. In order to decrease the heat output uncertainty, the temperature difference across the heat exchanger should be increased. This can be done by using a fluid with a lower specific heat or lowering the coolant flow rate. A more expensive solution that does not involve increasing the temperature difference is to use thermistors which are more accurate than thermocouples. It is also noted that the heat output uncertainty does not change greatly with heat input because the temperature difference in Eq.(2.15) is small compared to the third term which does not depend on heat input variation. One can conclude that only the measured 
heat input can be used to give reasonable results relating to heat transfer calculations requiring heat transfer as an input.

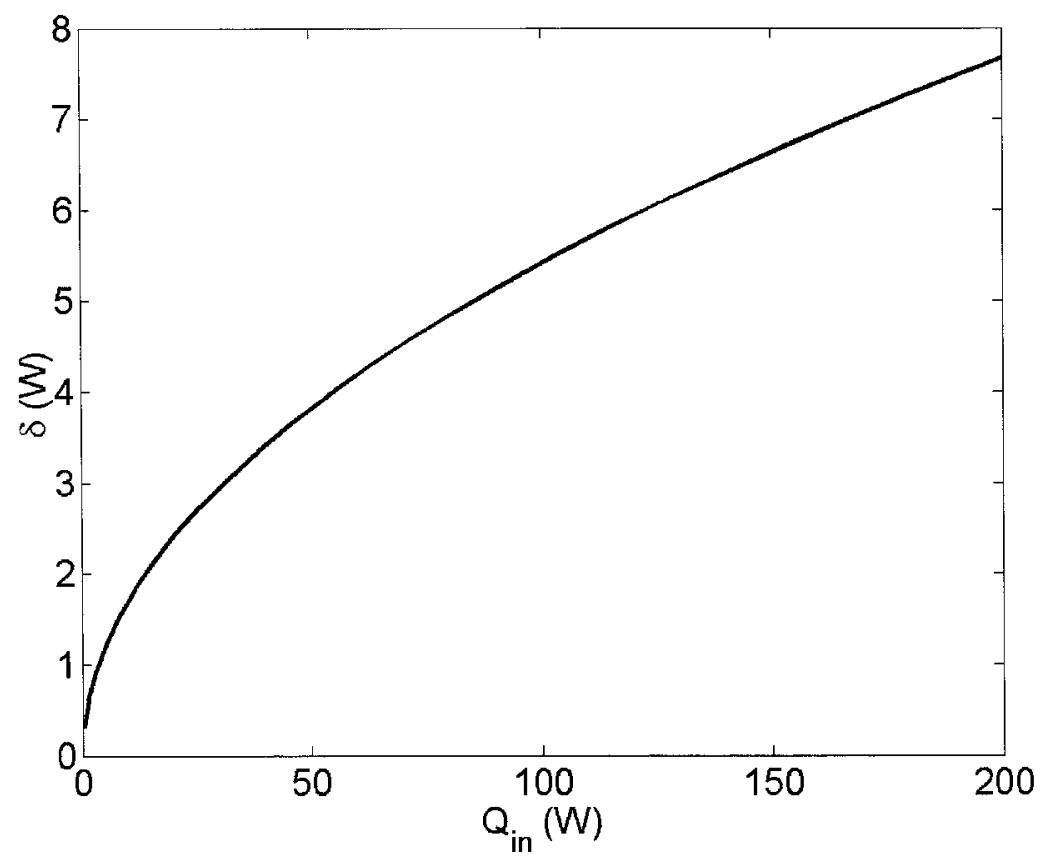

Figure 2.46: Heat Input Measurement Uncertainty

\subsubsection{Inclination Testing}

Adverse incline testing was performed with $5 \mathrm{~W}$ evaporator input and a sink temperature of $5^{\circ} \mathrm{C}$. Dryout was deemed to occur at $\psi=4^{\circ}$ for the AGHP and the AHP based on the evaporator temperature divergence seen in Figure 2.47 and Figure 2.48. Gravity assisted testing was preformed for the AHP and the AGHP with a favourable tilt angle of $\psi=-90^{\circ}$. Temperature variation in time and space results are seen in Figures 2.49, 2.51, 2.53, 2.55. Horizontal tests in Figures 2.50, 2.52, 2.54, 2.56 are shown for comparison. 


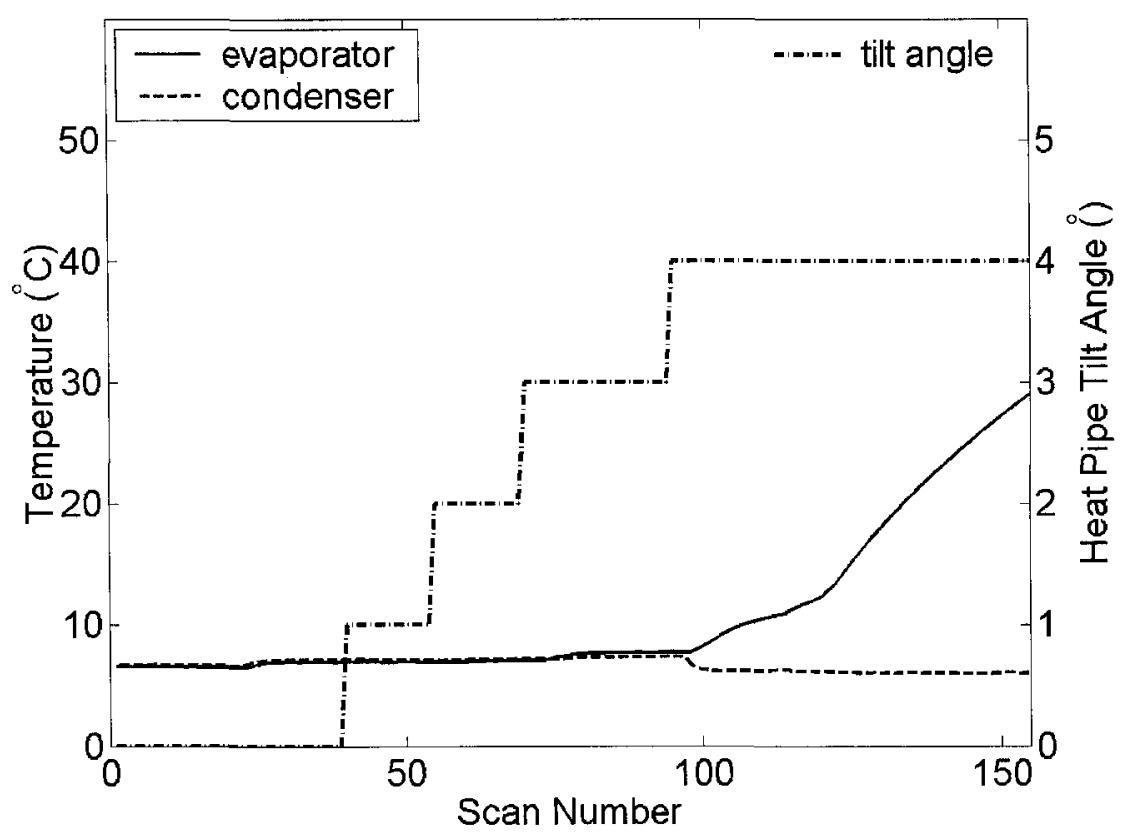

Figure 2.47: AGHP, Average Temperature, Source 5 W, Tilt Dryout

AGHP testing with $\psi=-90^{\circ}$ creating a favourable pressure gradient shows a smaller temperature difference between different heat pipe stations seen in Figure 2.49, compared to testing without inclination seen in Figure 2.50. The favourable pressure gradient accounts for friction and allows for a more nearly isothermal performance.

For the AHP, one can conclude from Figures 2.53, 2.54, 2.55, 2.56 that the temperature difference between the evaporator and condenser is greater with gravity assistance. Given the test with $\psi=-90^{\circ}$ was performed after all other tests, performance degradation is assumed. 


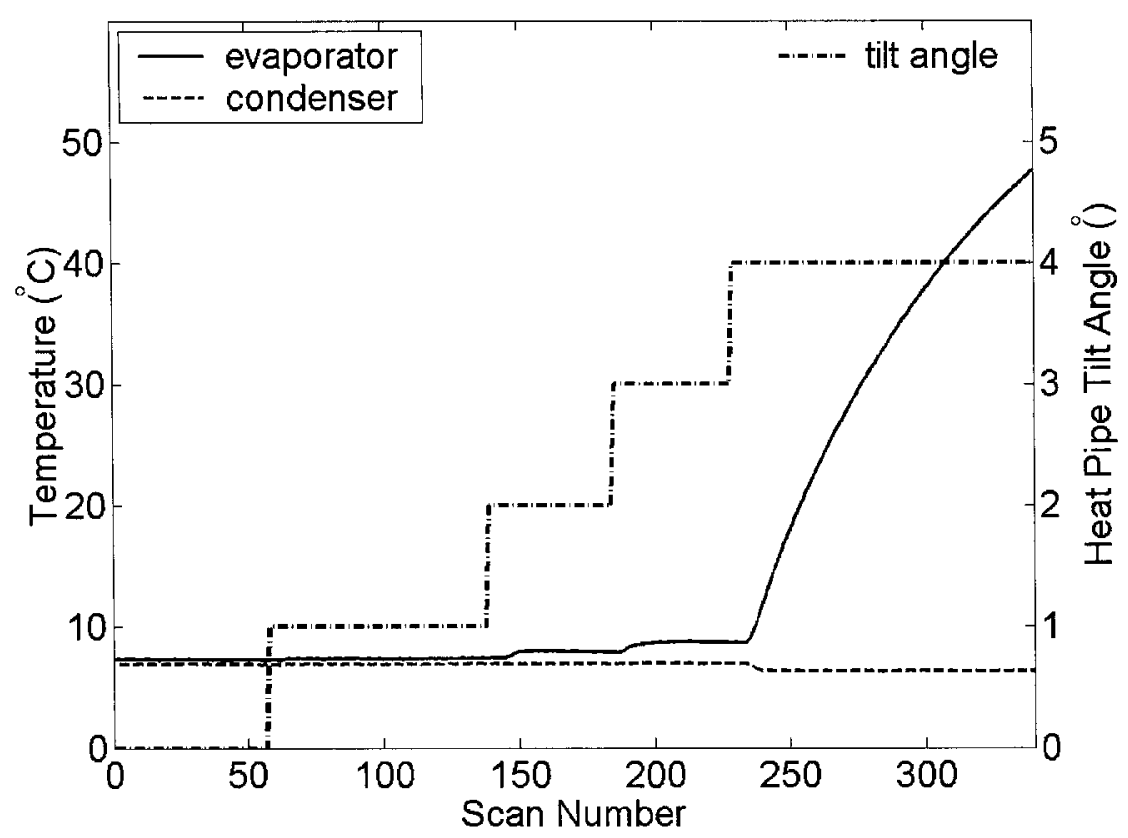

Figure 2.48: AHP, Average Temperature, Source 5 W, Tilt Dryout

\subsubsection{Horizontal Dryout}

AGHP and AHP dryout was observed at $190 \mathrm{~W}$ and $370 \mathrm{~W}$ and is shown in Figure 2.57 and Figure 2.58, respectively. It is interesting to note the departure from and return to steady-state for the AHP at $360 \mathrm{~W}$. This can be used as an indicator to dryout given a slight power increase. This was not observed with the AGHP. Comparison can be made using dryout data supplied by the manufacturer in Figure 2.59 and Figure 2.60 for the AGHP and AHP, respectively.

\subsubsection{AHP Heater Location Variation}

The artery inside the AHP is not symmetric with respect to heater location. It was of interest to see if the proximity of the heater to the artery would create a difference in performance with the heater above or beneath the evaporator. The possible testing 


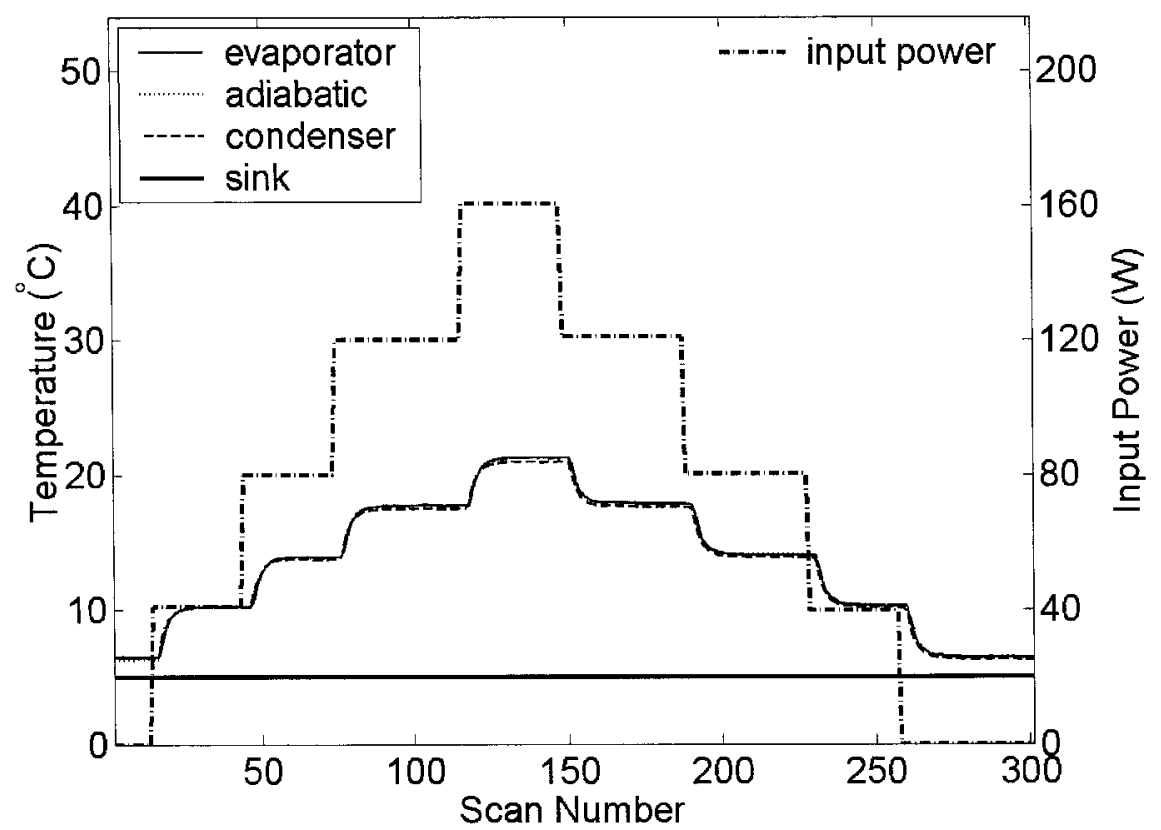

Figure 2.49: AGHP, Average Temperature, $\psi=-90^{\circ}$ tilt

scenarios are shown graphically using Figure 2.61. The heat pipe was placed with its artery at the bottom with respect to gravity based on the orientation markings given by the manufacturer. Comparison of performance with the heater below and above the evaporator is seen in Figure 2.62 and Figure 2.22, respectively. From observation, both orientations tend to give nearly equal results with respect to heater location. 


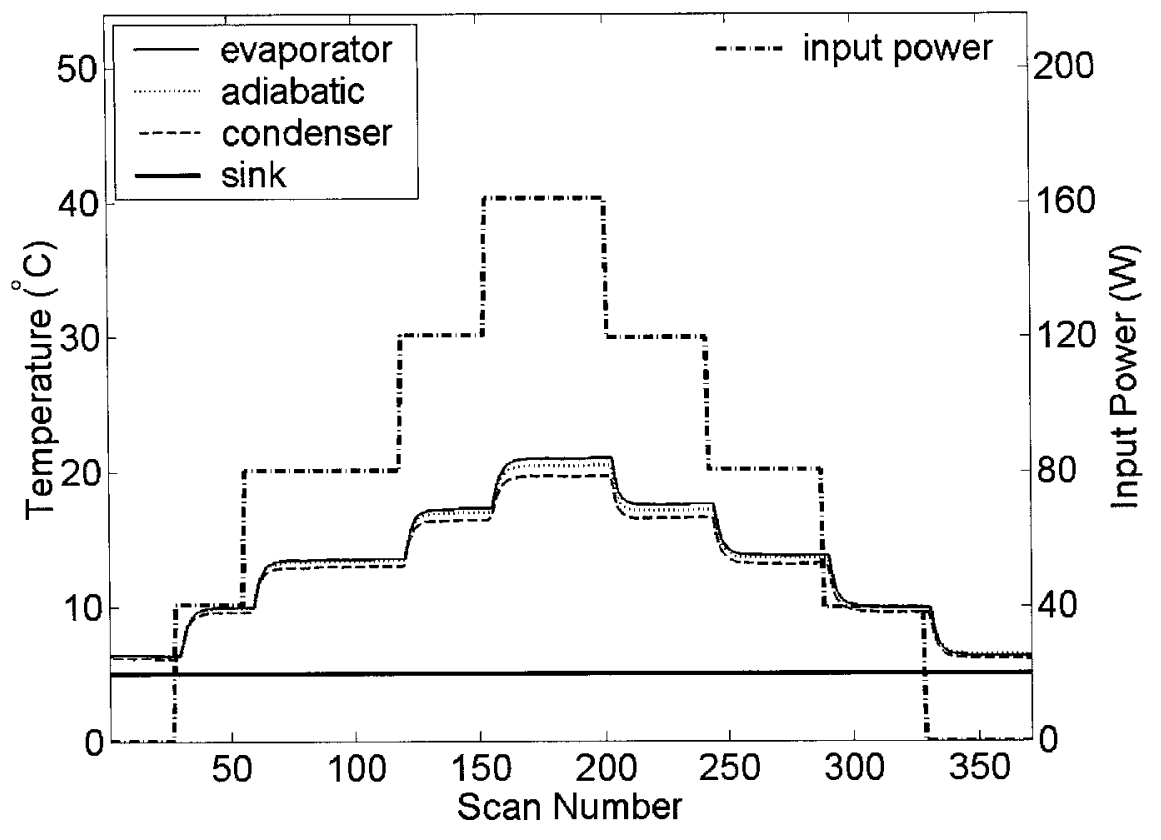

Figure 2.50: AGHP, Average Temperature, $\psi=0^{\circ}$ tilt

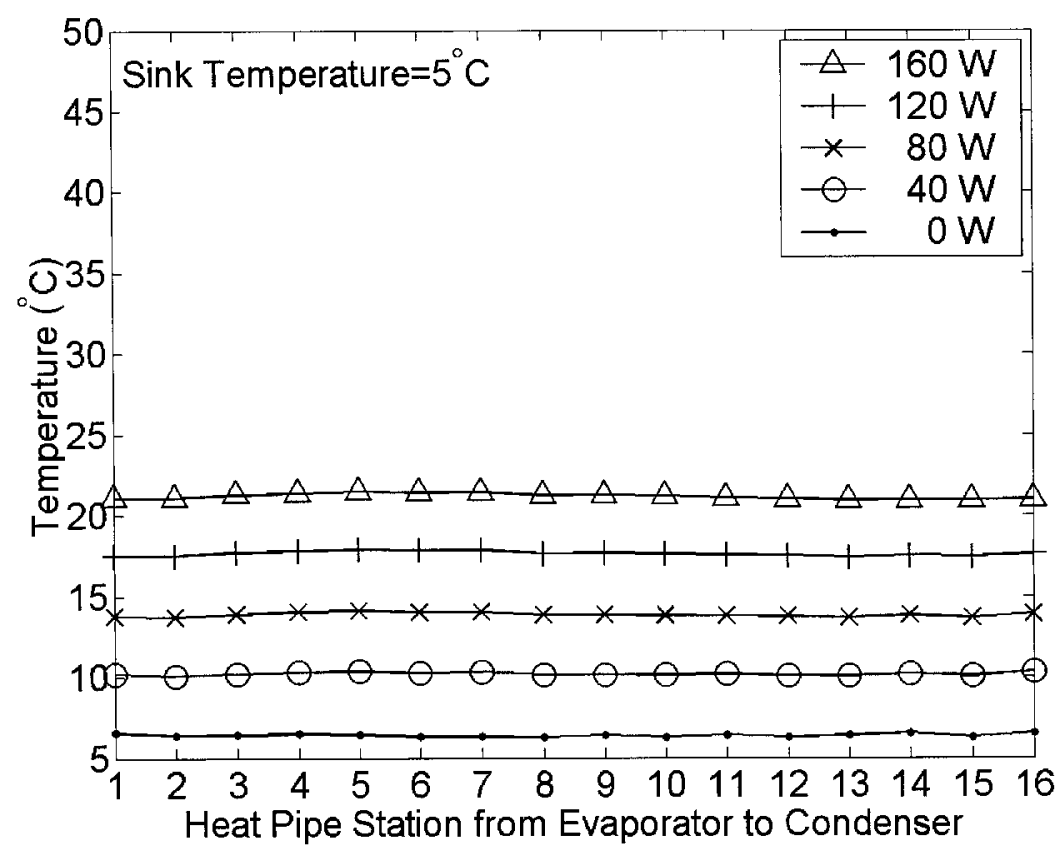

Figure 2.51: AGHP, Station Temperature, $\psi=-90^{\circ}$ tilt 


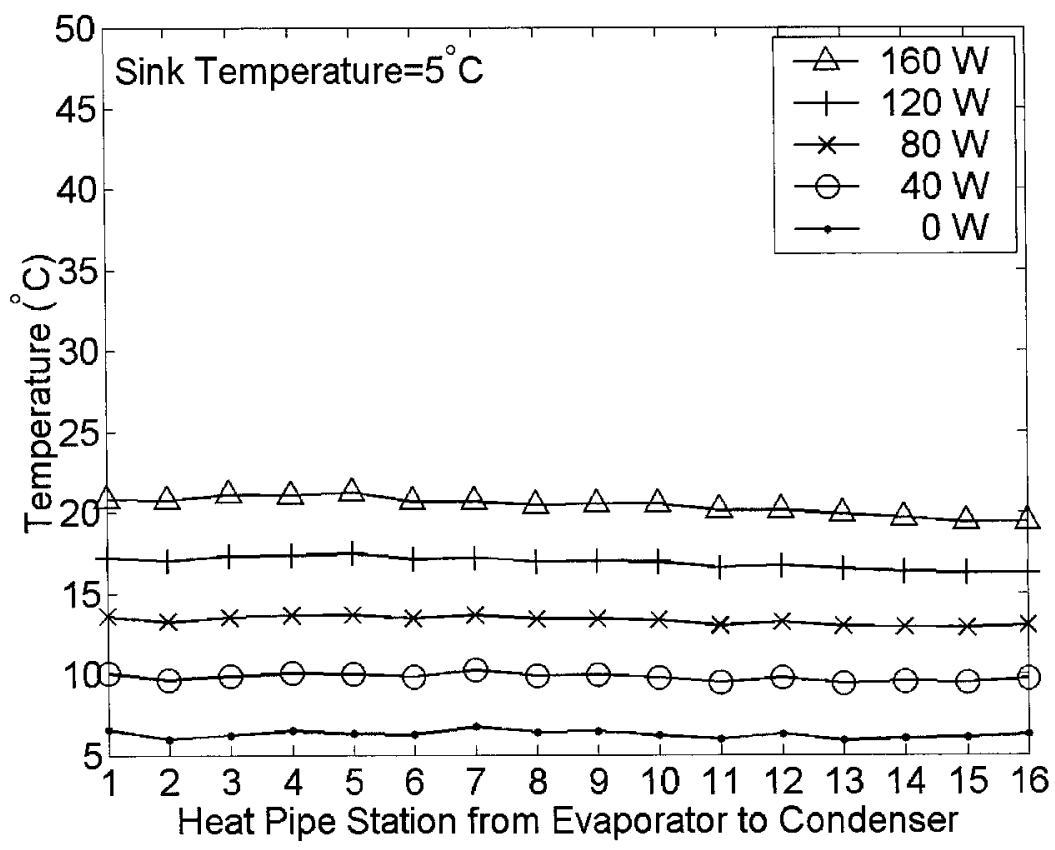

Figure 2.52: AGHP, Station Temperature, $\psi=0^{\circ}$ tilt

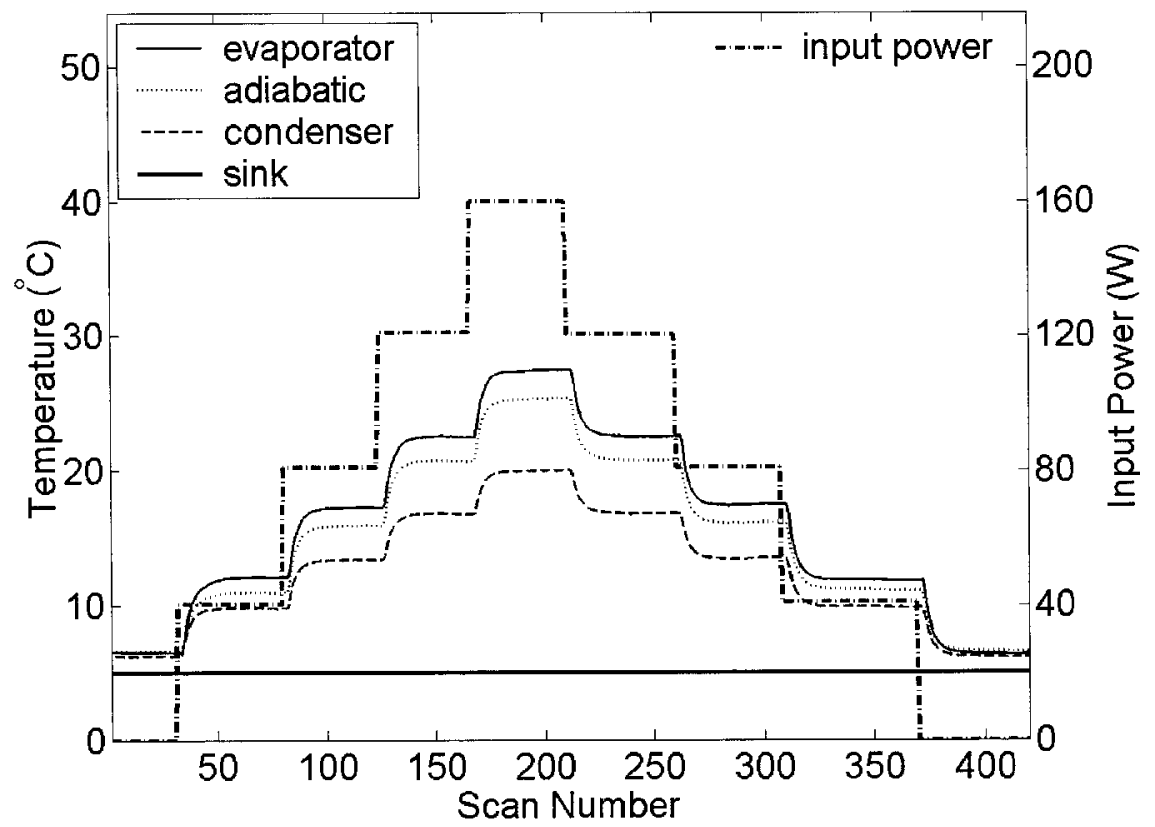

Figure 2.53: AHP, Average Temperature, $\psi=-90^{\circ}$ tilt 


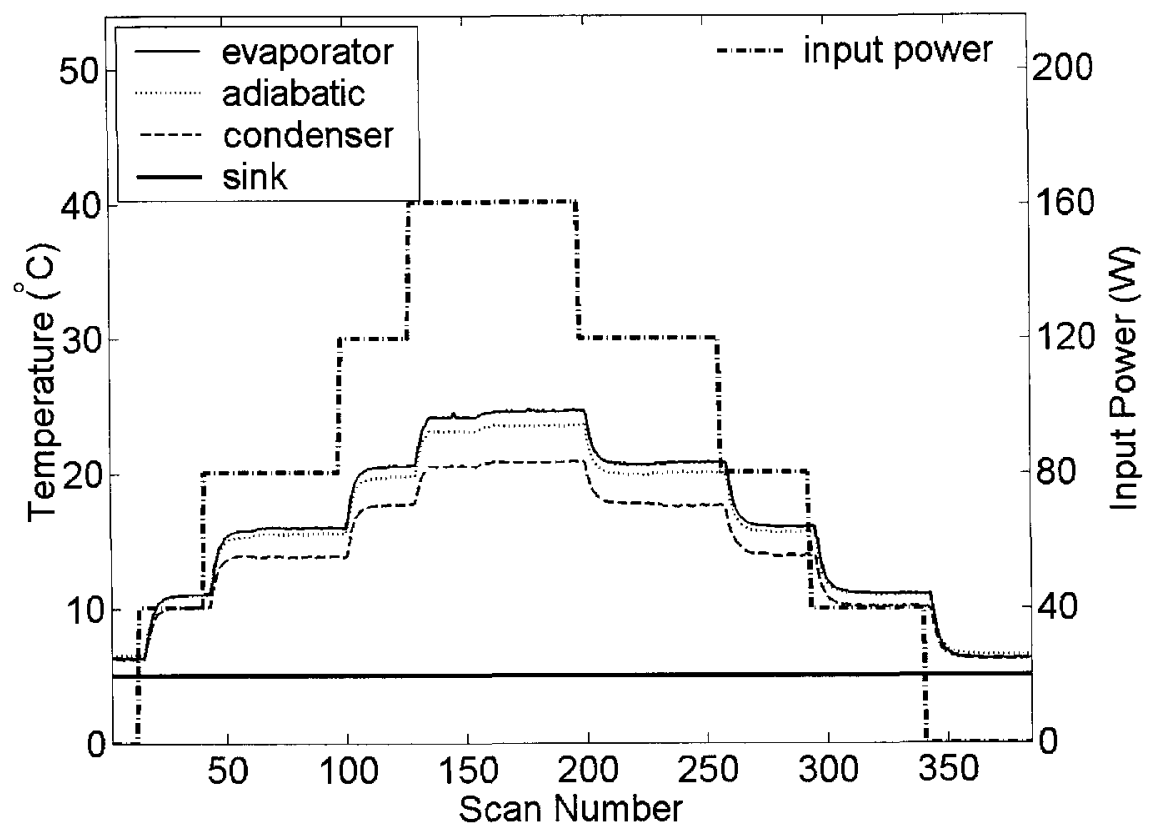

Figure 2.54: AHP, Average Temperature, $\psi=0^{\circ}$ tilt

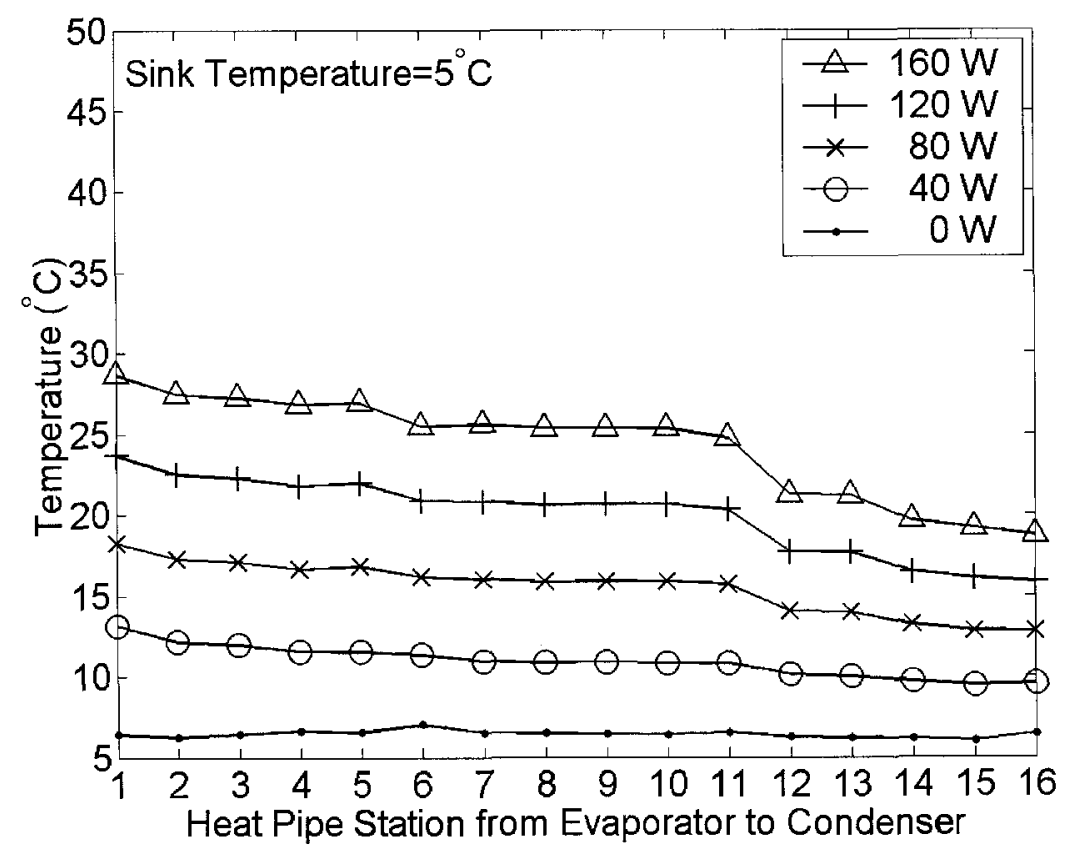

Figure 2.55: AHP, Station Temperature, $\psi=-90^{\circ}$ tilt 


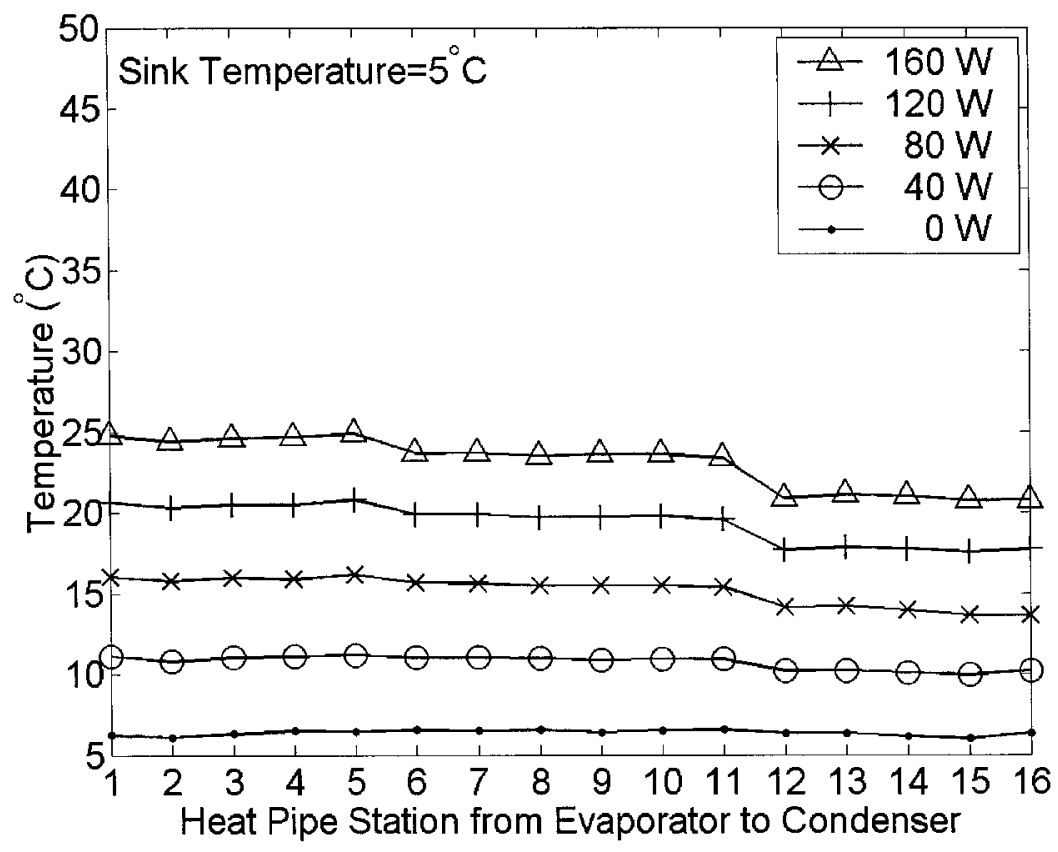

Figure 2.56: AHP, Station Temperature, $\psi=0^{\circ}$ tilt

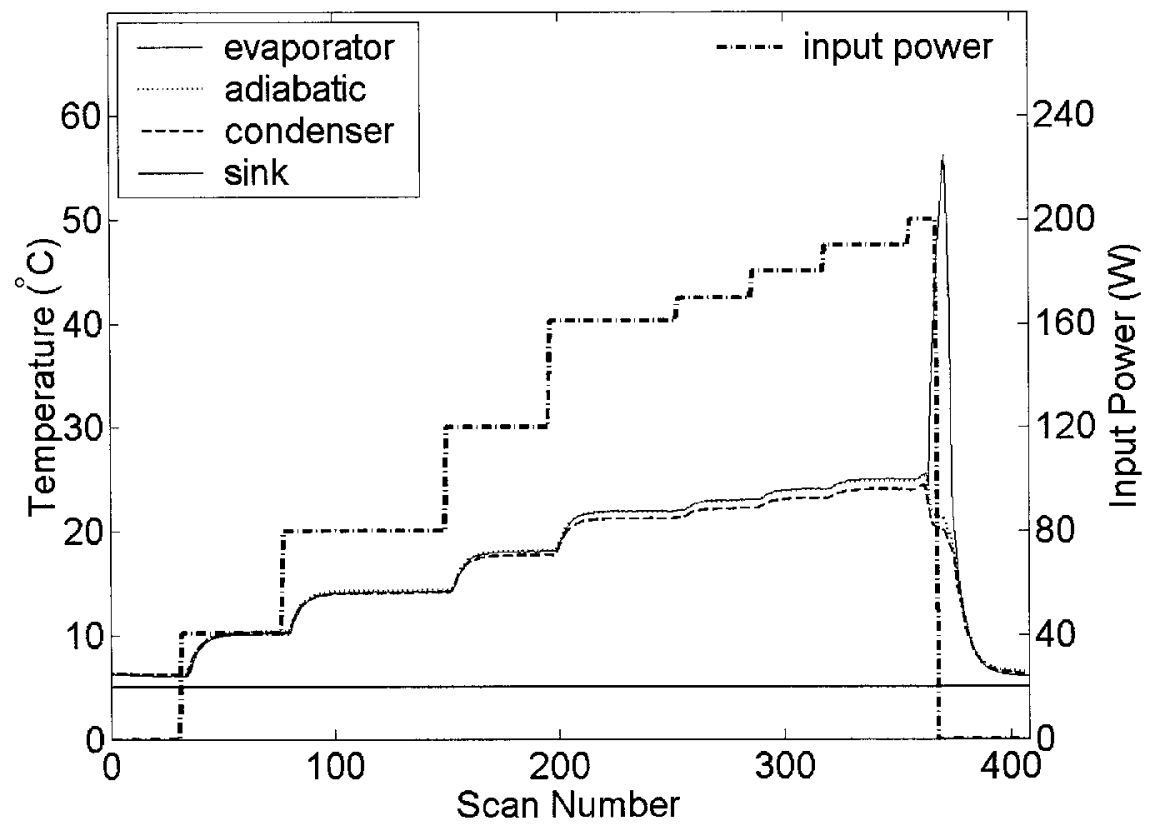

Figure 2.57: AGHP, Average Temperature, Horizontal Dryout 


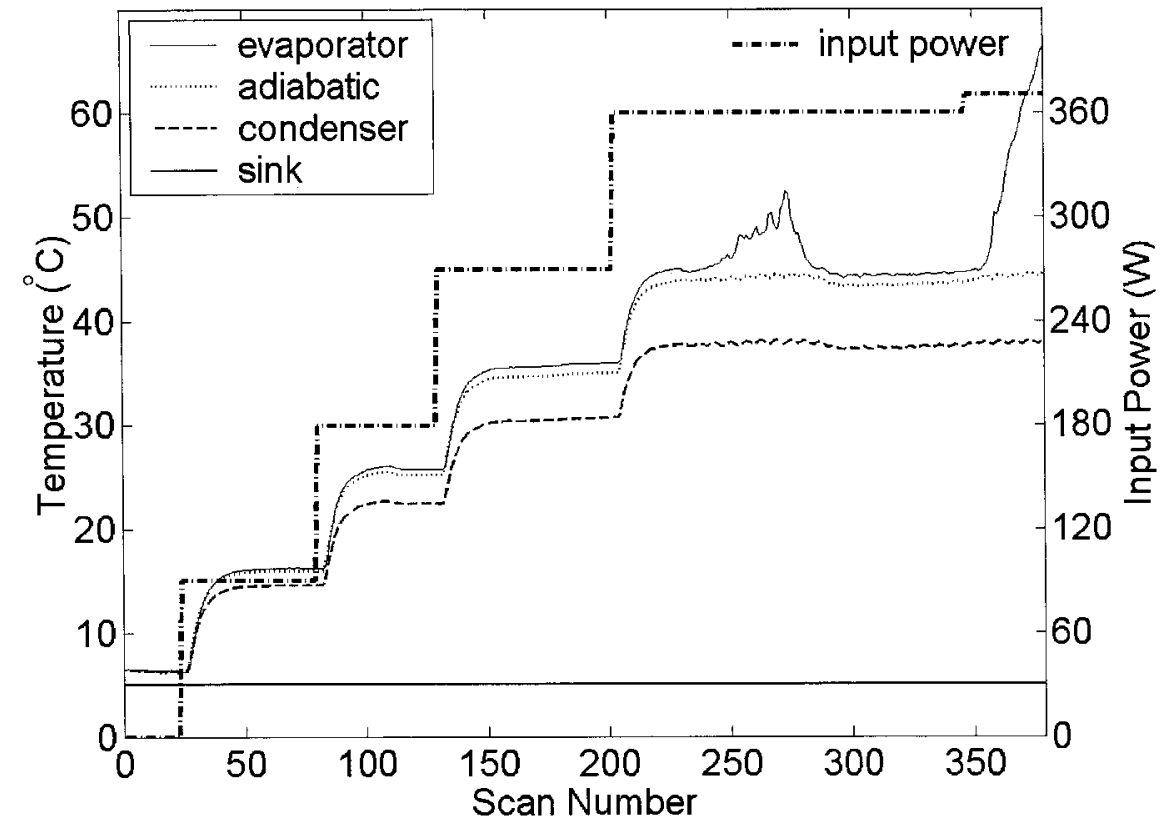

Figure 2.58: AHP, Average Temperature, Horizontal Dryout

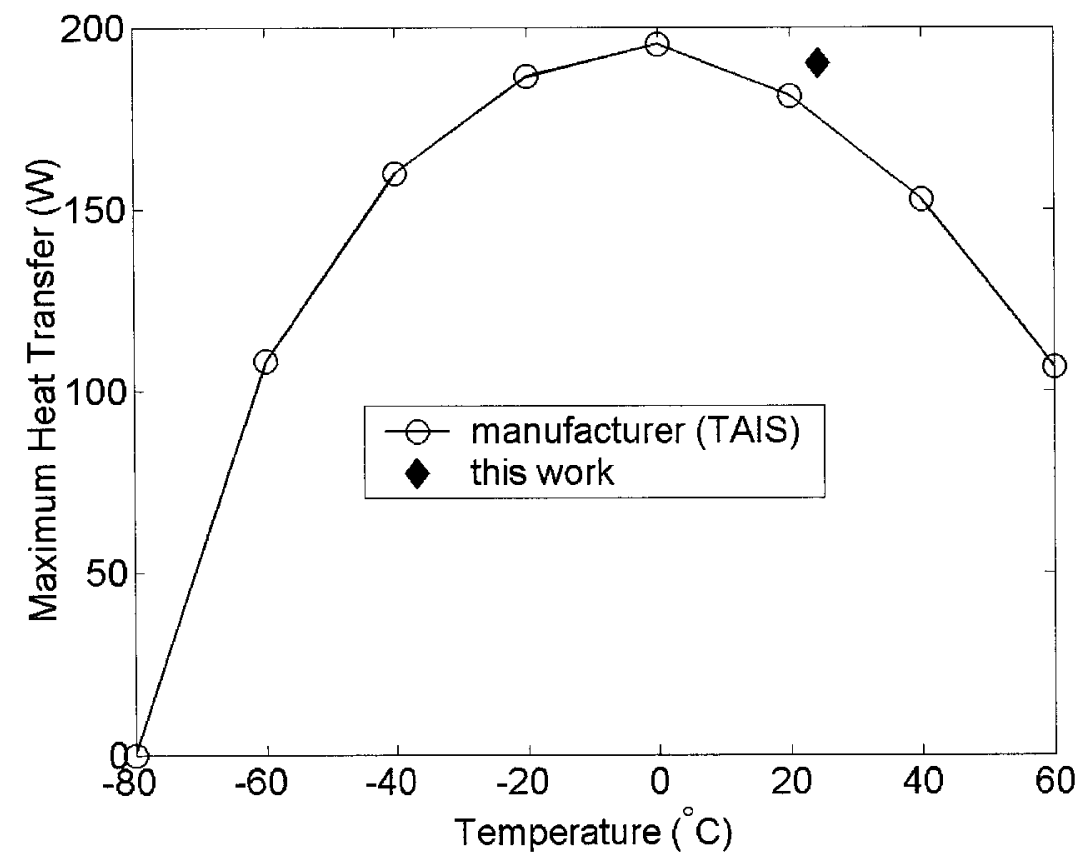

Figure 2.59: AGHP, Manufacturer Data Comparison 


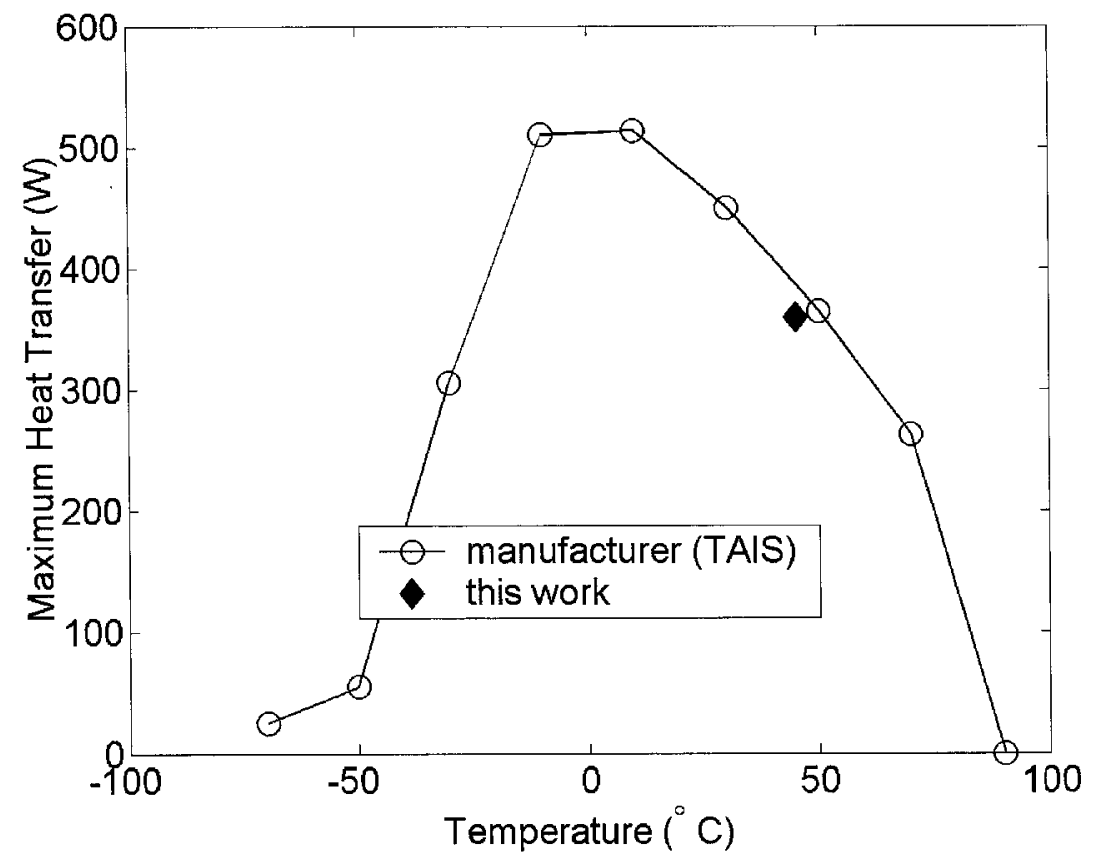

Figure 2.60: AHP, Manufacturer Data Comparison

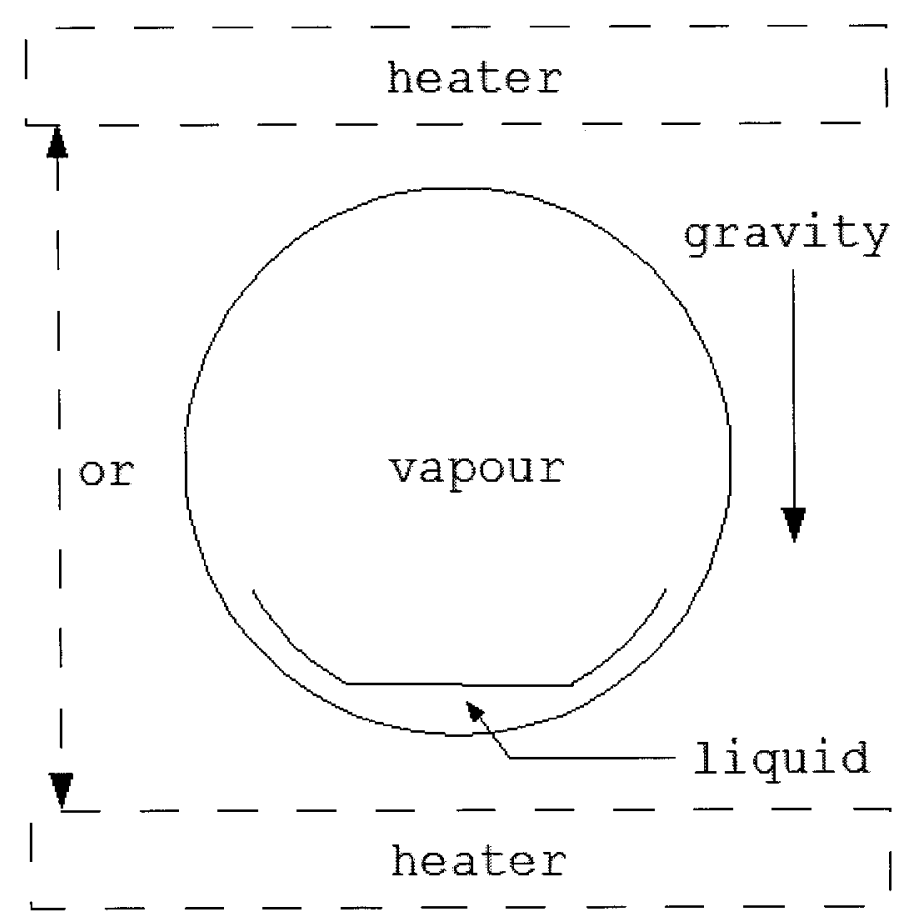

Figure 2.61: Possible AHP Heater Configurations 


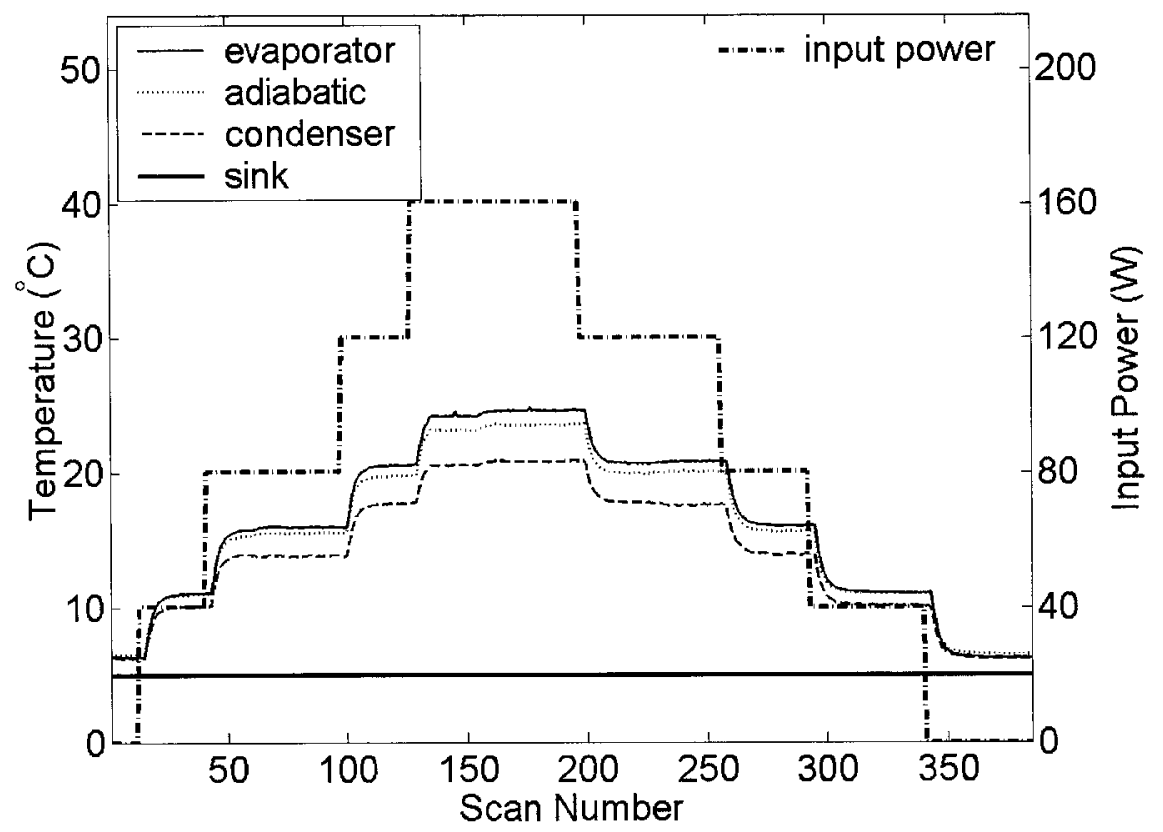

Figure 2.62: AHP, Average Temperature, Heater below Evaporator 


\section{Chapter 3}

\section{Numerical Modelling}

\subsection{Axially Grooved Heat Pipe Capillary Limit Theory}

The capillary pressure across the liquid-vapour interface can be approximated using the Laplace-Young equation, Eq.(3.1)

$$
P_{c}=\sigma\left(\frac{1}{R_{1}}+\frac{1}{R_{2}}\right)
$$

where $\sigma$ is the surface tension. A heat pipe's capillary transport limit is reached when the capillary pressure is at its theoretical maximum. The physical repercussion of this condition is penetration of the liquid flow by the vapour.

For an axially grooved heat pipe, choosing $R_{1}$ to be in the flow cross-sectional plane leads to $R_{2}=\infty$ based on the assumption of axially fully developed flow. For maximum capillary pressure with $R_{1}=\frac{w}{2 \cos (\theta)}$ based on the geometry of Figure 3.1 and Eq.(3.1), the contact angle $\theta$ must equal zero. 


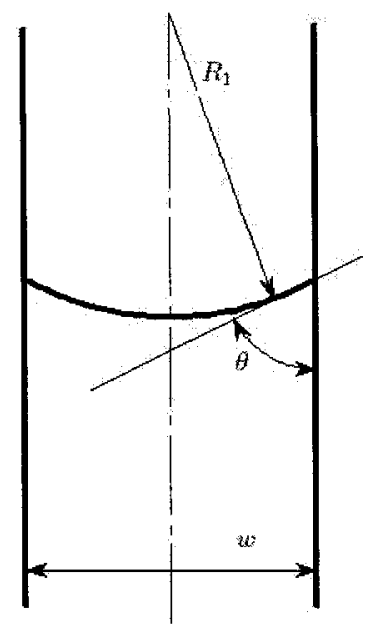

Figure 3.1: Axial Groove Meniscus

\subsection{Liquid Groove}

\subsubsection{Global Pressure Losses}

The axial liquid pressure change for steady-state flow is due to friction and body forces such as the axial component of gravity. The impact of inertial forces can be considered negligible due to the low liquid velocity. Noting that the heat pipe under question is curved, a liquid pressure gradient due to gravity will only be felt in line segments not axially perpendicular the earth's gravitational field. This is stated 
mathematically in Eq.(3.2) with the aid of Figure 3.2, where $\psi$ is the tilt angle.

$$
\left.\frac{d P_{l}}{d z}\right|_{\vec{g}}= \begin{cases}0 & \text { if } z \text { in } L_{1}, \\ -\rho_{l} \vec{g} \sin (\psi) \sin (\gamma) & \text { if } z \text { in } L_{2}, \\ -\rho_{l} \vec{g} \sin (\psi) & \text { if } z \text { in } L_{3}, \\ -\rho_{l} \vec{g} \sin (\psi) \sin (\gamma) & \text { if } z \text { in } L_{4}, \\ 0 & \text { if } z \text { in } L_{5}\end{cases}
$$

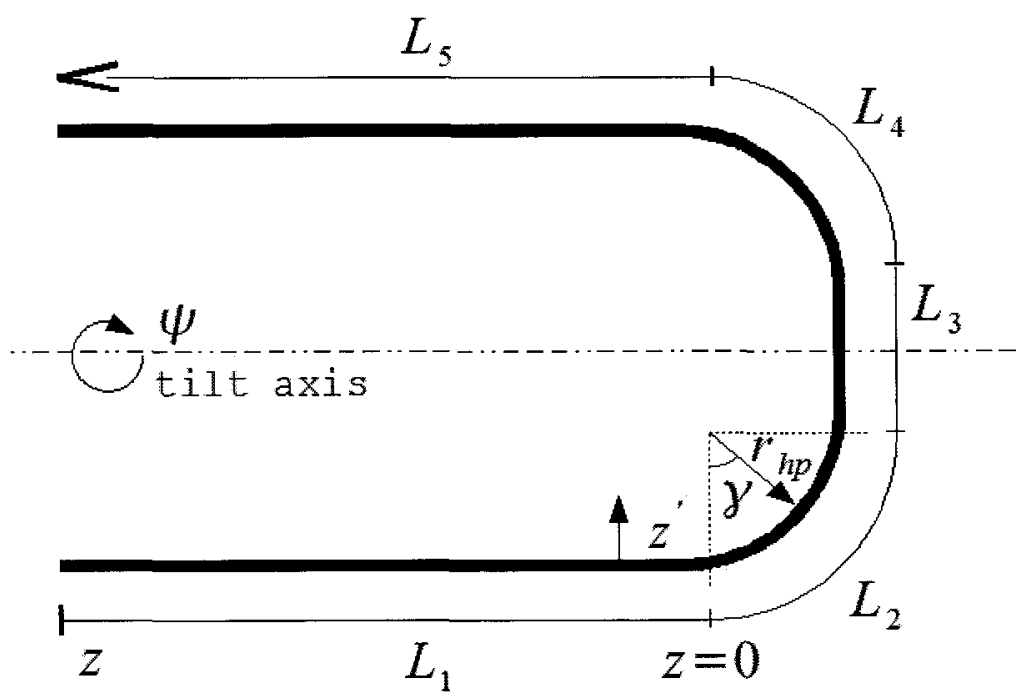

Figure 3.2: Gravitational Influence on Line Segments

Deriving the hydrostatic pressure gradient for the curved segments of the heat pipe, i.e., $L_{2}$ and $L_{4}$ here involves the introduction of a new coordinate $z^{\prime}$ as seen in Figure 3.2. The following trigonometric and differential relations are obtained in 
Eqs.(3.3 - 3.6).

$$
\begin{aligned}
z & =r_{h p} \gamma \\
z^{\prime} & =r_{h p}(1-\cos (\gamma)) \\
\frac{\partial z^{\prime}}{\partial z} & =\sin (\gamma) \\
\left.\frac{\partial P_{l}}{\partial z}\right|_{\vec{g}} & =\left.\frac{\partial P_{l}}{\partial z^{\prime}}\right|_{\vec{g}} \frac{\partial z^{\prime}}{\partial z} \\
& =-\rho \vec{g} \sin (\psi) \sin (\gamma)
\end{aligned}
$$

One may integrate to find the pressure change over the entire curved section. Noting $\gamma=\frac{z}{r_{h p}}$ leads to Eq.(3.7)

$$
\begin{aligned}
\left.\Delta P_{l, L_{2,4}}\right|_{\vec{g}} & =-\rho \vec{g} \sin (\psi) \int_{0}^{\frac{\pi r}{2}} \sin \left(\frac{z}{r_{h p}}\right) d z \\
& =-\left.\rho \vec{g} \sin (\psi)\left[-r_{h p} \cos \left(\frac{z}{r_{h p}}\right)\right]\right|_{0} ^{\frac{\pi r}{2}} \\
& =-r_{h p} \rho \vec{g} \sin (\psi)
\end{aligned}
$$

With two curved components and one straightaway, the total pressure drop due to gravity is stated in Eq.(3.8). Although the result for the total pressure change in Eq.(3.7) could have been obtained easily without the explicit derivation, the advantage of knowing the change in pressure at any station along the heat pipe could be used in local modelling.

$$
\left.\Delta P_{l}\right|_{\vec{g}}=-\rho_{l} \vec{g} \sin (\psi)\left(2 r_{h p}+L_{3}\right)
$$


It is common in fluid mechanics to use the hydraulic radius defined in Eq.(3.9) as the characteristic length in the non-dimensional analysis of conduit flow.

$$
r_{h l}=\frac{2 A_{l}}{P}
$$

Conduit flow is similar to flow in the liquid groove of a heat pipe, excluding the behaviour at the liquid-vapour interface.

\subsubsection{Groove Modelling}

A sample length of heat pipe extruded through the same die as the tested AGHP was used to obtain an accurate representation of the groove contour. Different methods were tried to magnify the groove for ease of measurement. An overhead projector and an HP-PSC-1310 digital scanner gave poor resolution and were abandoned. Eventually, a Mitutoyo profile projector, type PJ-300 seen in Figure 3.3 was used to measure the axial groove heat pipe cross-section coordinates at every 0.001 in $(0.0254 \mathrm{~mm})$. The groove chosen for measurement from the seventeen available was the only one having its axis of symmetry coincident with the axis of symmetry of the heat pipe cross-section as seen in Figure 3.4. This groove was chosen because the orthogonal heat pipe saddle wall could be used as a guide when attempting to make the groove's axis of symmetry parallel to one of the profile projector's travel directions. The projector measurement accuracy was given as 0.0001 in $(0.00254$ $\mathrm{mm}$ ). A numerical grid was then created with the boundary along the projection profile.

The 72 outline points measured were fit to a 6th degree polynomial with Matlab's polyfit function which fits the data using a least-squares approach. The polynomial 


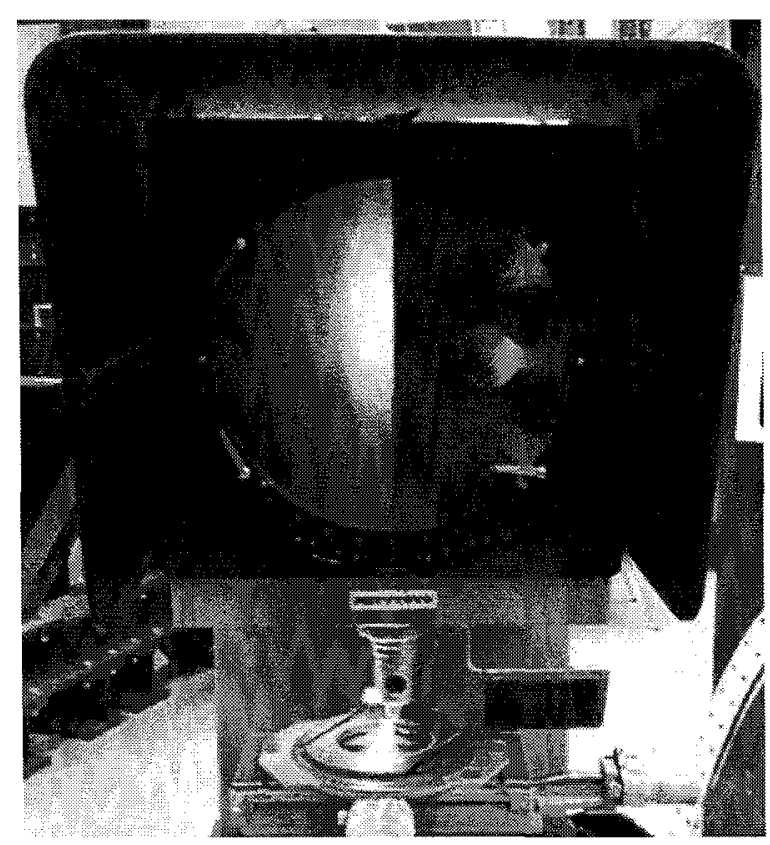

Figure 3.3: Mitutoyo Profile Projector

fit serves the purpose of smoothing out the discontinuities measured at a particular cross-section caused partially by imperfections created when the cross-section sample was cut. The natural jaggedness associated with a particular cross-section is considered random and would disappear if one were to take the average of an infinite number of cross-sections constituting the conduit. Another convenience obtained when fitting the data to a polynomial is the ability to differentiate a point of the outline analytically. This ability is useful when calculating contact angles. A slight modification was made to the first and the last point such that the axial symmetry condition could be met since some information is lost when the data is fit to a polynomial. A constant slope was forced on both endpoints to obtain symmetry. Comparison of the 6th order fit and the points measures at every thousandth of an inch is seen in Figure 3.5. Normalization of the groove coordinates by its length $L_{g}=0.071 \mathrm{in}(1.80 \mathrm{~mm})$ is performed with Eq.(3.10) and Eq.(3.11). All numerical 


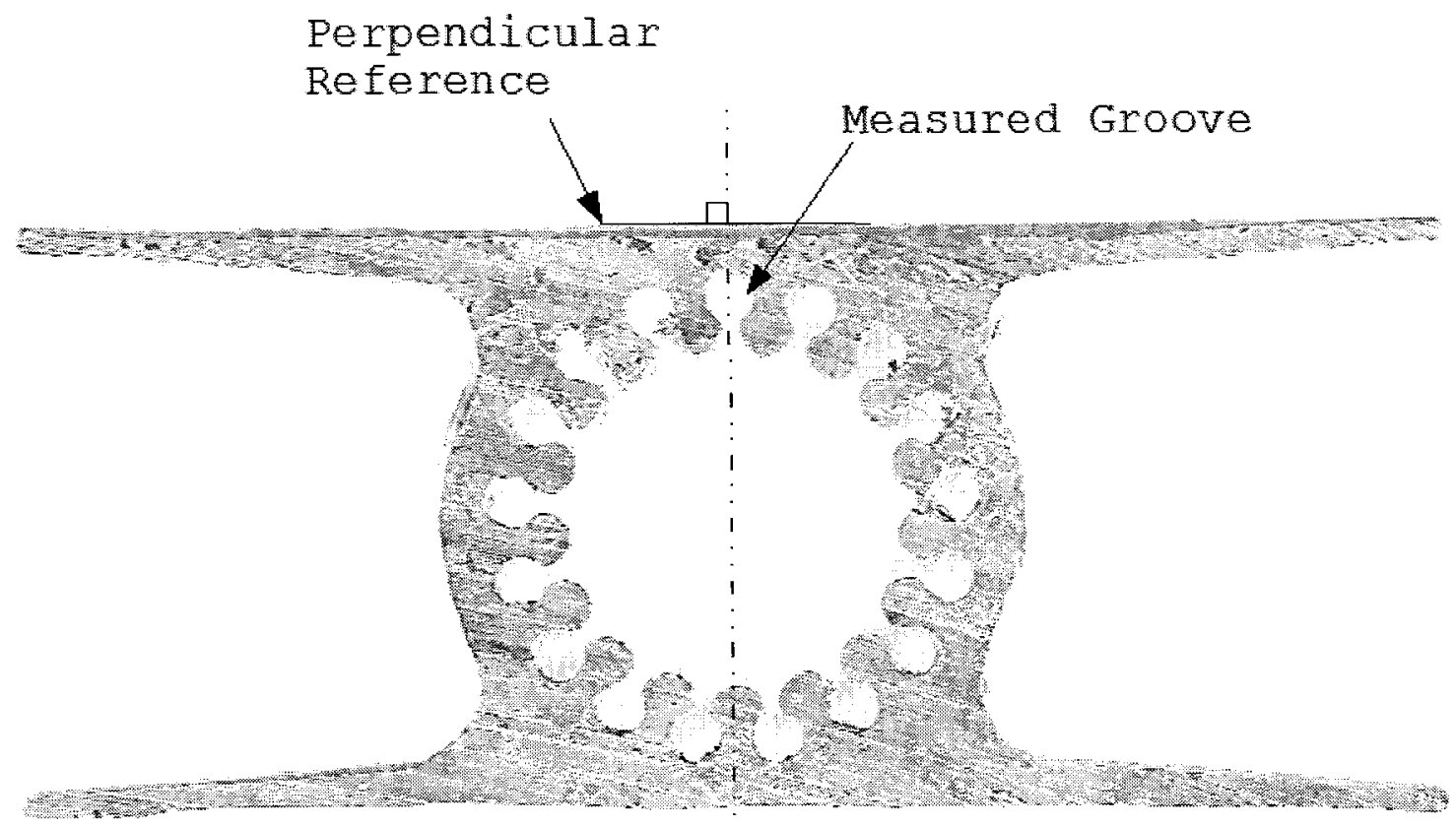

Figure 3.4: AGHP Cross-Section Used for Measurement

work with respect to the liquid groove will use this convention.

$$
\begin{gathered}
x^{*}=\frac{x}{L_{g}} \\
y^{*}=\frac{y}{L_{g}}
\end{gathered}
$$

The groove area was calculated by numerically integrating the digital map of the groove contour using the trapezoid rule for the area between the axis of symmetry and groove wall as seen in Eq.(3.12). The part of the perimeter touching the groove wall was calculated by adding the lengths of all line segments connecting adjacent groove outline nodes using the pythagorean theorem, Eq.(3.13). Note the division 


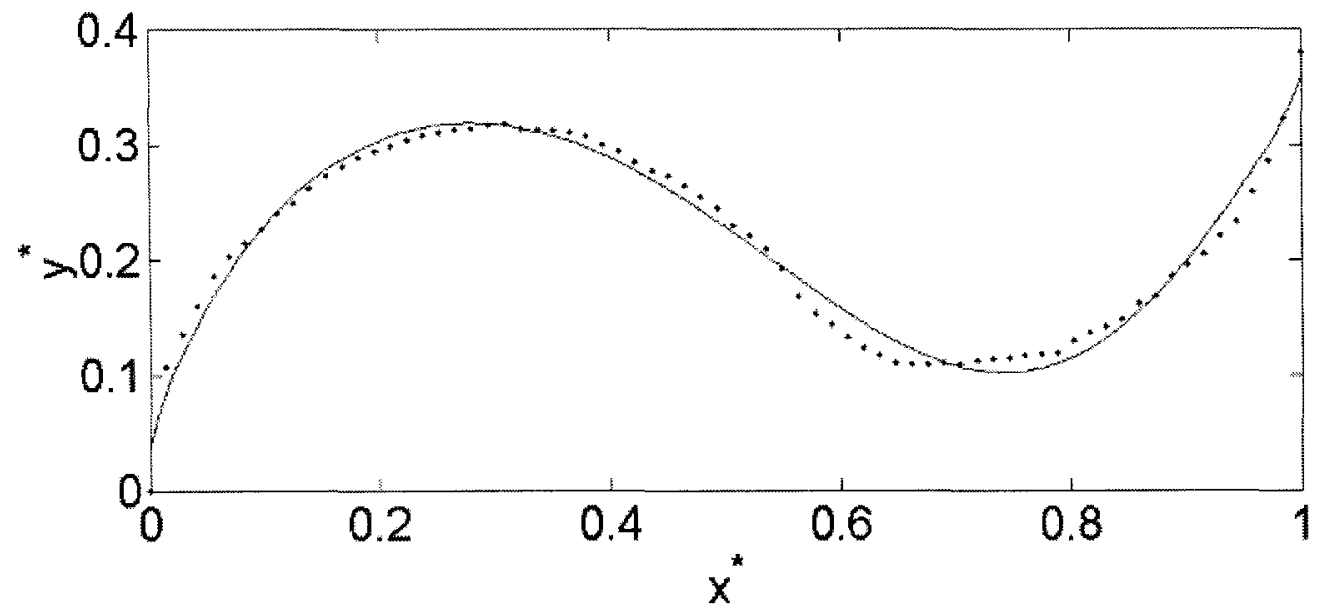

Figure 3.5: Curve Fit to Measured Points

by two since only half the groove is integrated.

$$
\begin{gathered}
\frac{A_{l}}{2}=\sum_{i=0}^{i_{\text {end }}} \frac{1}{2}\left(y_{i+1}+y_{i}\right)\left(x_{i+1}-x_{i}\right)-A_{d} \\
\frac{P}{2}=\sum_{i=0}^{i_{\text {end }}} \sqrt{\left(x_{i+1}-x_{i}\right)^{2}+\left(y_{i+1}-y_{i}\right)^{2}}+L_{i n t}
\end{gathered}
$$

The area deficit $A_{d}$, is defined in this work as the liquid cross-sectional area lost from curving the interface and is seen surrounded by dashed lines in Figure 3.6. It is subtracted from the integrated area using geometric relations obtained with knowledge of the contact angle and the slope of the groove at the attachment point.

The range in location of the attachment point and magnitude of the contact angle gives four general geometric configurations leading to the development of four slightly different mathematical formulations used to calculate the area deficit. The four configurations are seen in Figure 3.7 , where $\lambda$ is the groove slope tangent an- 


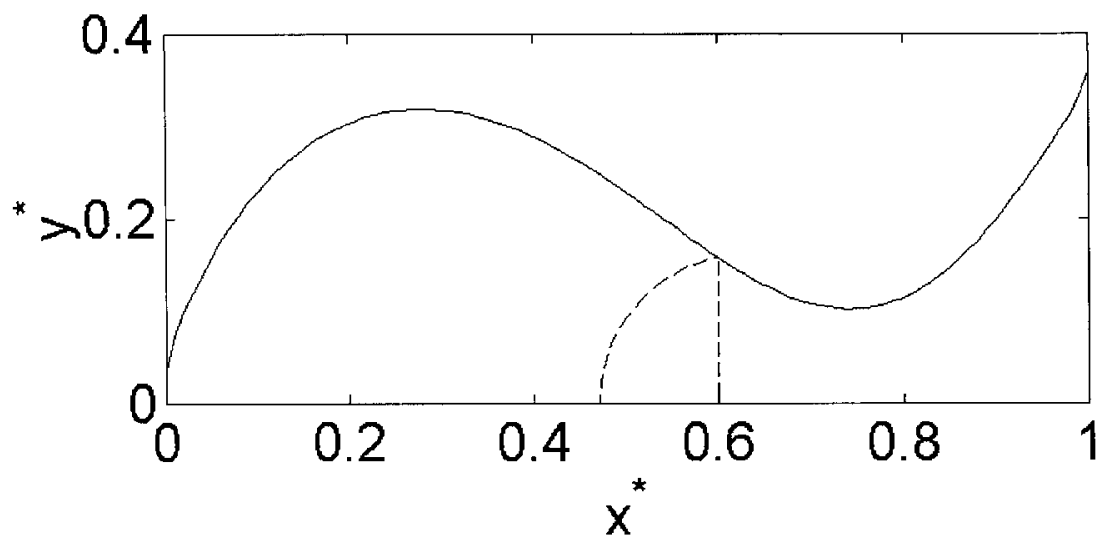

Figure 3.6: Outlined Area Deficit

gle at the attachment point and $\theta$ is the contact angle. These angles can be seen specifically for Case 2 in Figure 3.8. The angle $\sigma_{R}$ is made by the interfacial radius of curvature crossing the attachment point and the groove axis of symmetry. The term $\sigma_{i}$ represents the varying angle made between the radius of curvature and axis of symmetry as one draws the liquid-vapour interface curve. The resultant $x$ and $y$ coordinates of the interface are functions of the sweep angle. All cases are generalized in Tab.(3.1).

Once $\sigma_{R}$ is determined, one can solve for the interfacial radius of curvature $R$ and horizontal distance between the attachment point and the centre of curvature $h$, using Eq.(3.14) and Eq.(3.15), respectively.

$$
\begin{gathered}
R=\frac{w}{2 \sin \left(\sigma_{R}\right)} \\
h=\sqrt{R^{2}-\left(\frac{w}{2}\right)^{2}}
\end{gathered}
$$

Cases 1,3 , and 4 can be described using geometric constructions similar to the one 

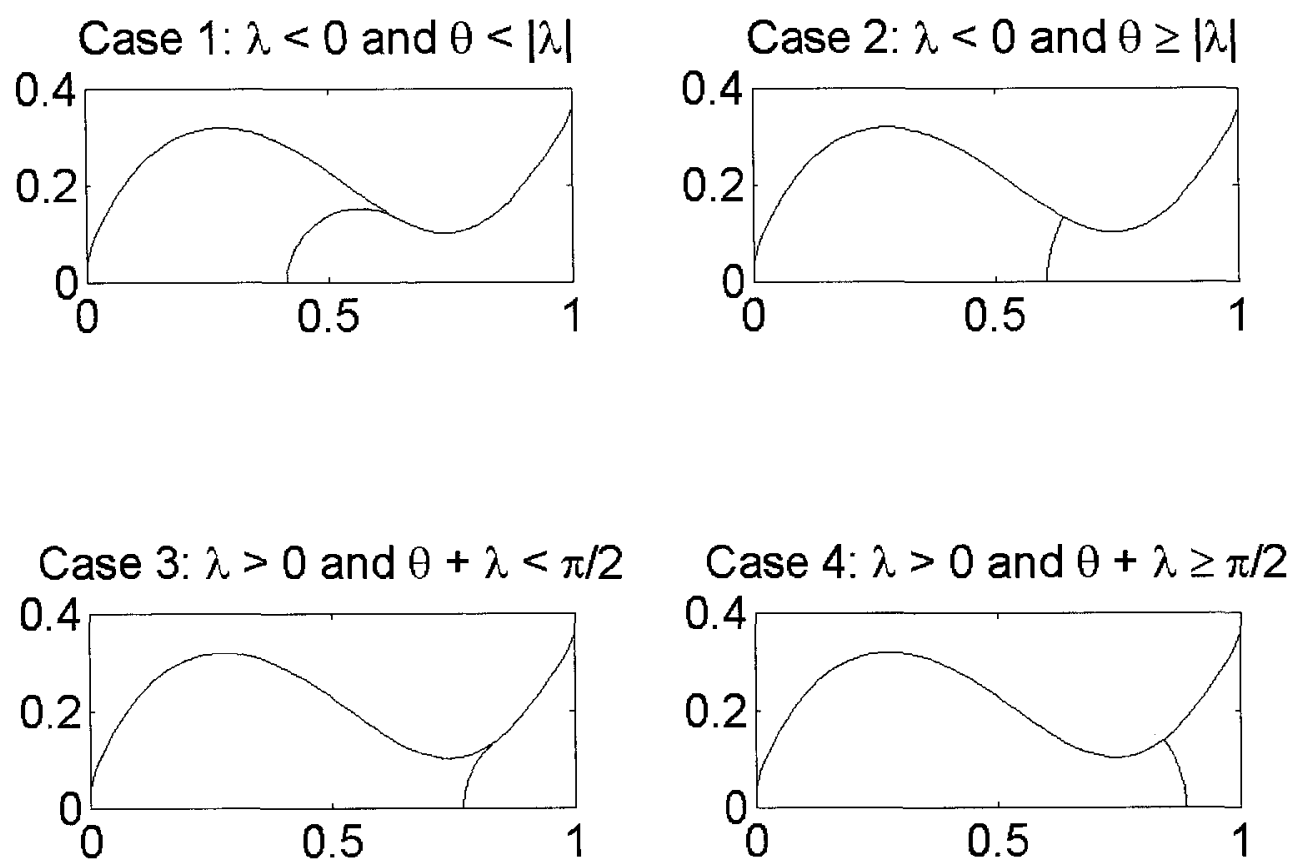

Figure 3.7: Four Interfacial Cases

applied to Case 2. Case 4 is physically unreasonable since it suggests that the liquid pressure is greater than the vapour pressure at that particular cross-section. This would not allow for the heat transfer cycle to occur given ammonia as a working fluid. Case 4 is introduced due to its mathematical feasibility and is encountered during parametrization, but the results are ignored. The area deficit can by found using the arc area formed by the interfacial radius of curvature and the area of the right angle triangle formed by $\mathrm{R}, \mathrm{h}$, and $\frac{w}{2}$ from Figure 3.8. The liquid-vapour interface length, $L_{\text {int }}$ can be found using $\mathrm{R}$ and $\sigma_{R}$ from Figure 3.8. The area deficit can be subtracted from the area between the groove wall and axis of symmetry to obtain 


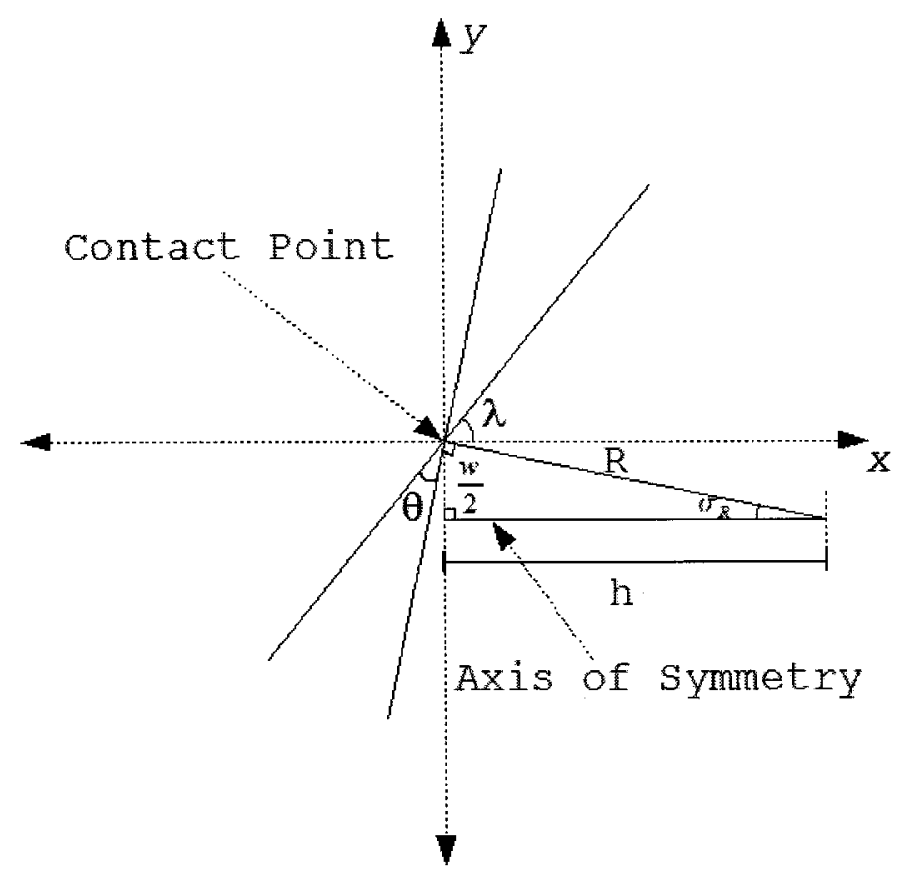

Figure 3.8: Angle Layout for Case 2

the area of the half-groove. The interface length is added to the length of the groove wall to obtain the perimeter of the half-groove.

No model is used to approximate the contact wetting angle based on the flow physics. A parametric variation of the wetting angle can be performed to evaluate the effect of contact angle on Poiseuille number and eventually the overall heat transfer.

\subsection{Governing Equation}

Axial groove flow behaviour of a given heat pipe is needed for accurate modelling. The continuity and momentum equations for fully developed incompressible flow reduce to the Poisson equation, Eq.(3.16) and will be taken as the governing equation 
Table 3.1: Four Interfacial Cases

\begin{tabular}{|c|c|c|c|c|}
\hline & Case 1 & Case 2 & Case 3 & Case 4 \\
\hline$\sigma_{R}$ & $|\lambda|-\theta+\frac{\pi}{2}$ & $-|\lambda|+\theta+\frac{\pi}{2}$ & $\lambda+\theta-\frac{\pi}{2}$ & $-\lambda-\theta+\frac{\pi}{2}$ \\
$A_{d}$ & $\frac{\pi-\sigma_{R}}{2} R^{2}+\frac{h w}{4}$ & $\frac{\sigma_{R}}{2} R^{2}-\frac{h w}{4}$ & $\frac{\sigma_{R}}{2} R^{2}-\frac{h w}{4}$ & $-\frac{\sigma_{R}}{2} R^{2}+\frac{h w}{4}$ \\
$L_{\text {int }}$ & $R\left(\pi-\sigma_{R}\right)$ & $R\left(\sigma_{R}\right)$ & $R\left(\sigma_{R}\right)$ & $R\left(\sigma_{R}\right)$ \\
$x\left(\sigma_{i}\right)$ & $-h+R \cos \left(\sigma_{i}\right)$ & $h-R \cos \left(\sigma_{i}\right)$ & $h-R \cos \left(\sigma_{i}\right)$ & $-h+R \cos \left(\sigma_{i}\right)$ \\
$y\left(\sigma_{i}\right)$ & $R \sin \left(\sigma_{i}\right)$ & $R \sin \left(\sigma_{i}\right)$ & $R \sin \left(\sigma_{i}\right)$ & $R \sin \left(\sigma_{i}\right)$ \\
\hline
\end{tabular}

for the liquid flow.

$$
\frac{\partial^{2} u}{\partial x^{2}}+\frac{\partial^{2} u}{\partial y^{2}}=\frac{1}{\mu_{l}} \frac{d P_{l}}{d z}
$$

Closed form solutions of Eq.(3.16) exist for simple geometries Ref.[7], but numerical methods become preferable when faced with more complicated cross-sections.

Relevant dimensionless parameters include the Reynolds number in Eq.(3.17) and the friction factor in Eq.(3.18). These combine to form the Poiseuille number in Eq.(3.19).

$$
\begin{gathered}
R e_{l}=\frac{2 r_{h l} \rho_{l} \bar{u}_{l}}{\mu_{l}} \\
f_{l}=\frac{2 \tau_{l}}{\rho_{l} \bar{u}_{l}^{2}} \\
P o_{l}=R e_{l} f_{l}
\end{gathered}
$$


The Poiseuille number is obtained numerically when solving for the nondimensional velocity field using the governing equation. The flow remains steady from balancing the wall shear and the fluid pressure change as seen in Figure 3.9 and Eq.(3.20).

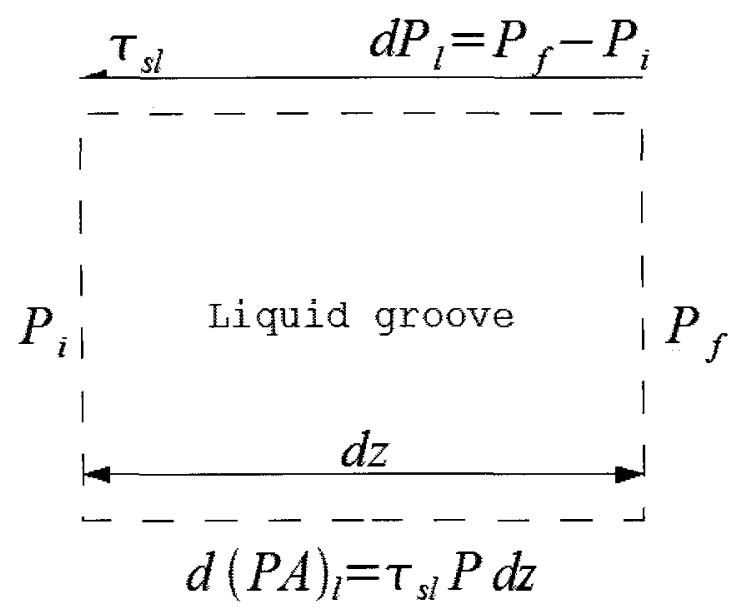

Figure 3.9: Balance Between Pressure and Shear

$$
\frac{d P_{l}}{d z}=\tau_{\mathrm{sl}} \frac{P}{A_{l}}
$$

The significance of the Poiseuille number is its independence with respect to the pressure gradient. A general solution can thus be obtained that is uniquely dependent on the groove geometry.

It should be noted that the balance of shear and pressure forces is valid only when the entire groove is bound by the no-slip condition. The liquid flow will be modelled as having a vapour counterflow boundary condition, reducing the validity of assumed wall shear and pressure difference balance. Given that the portion of the groove boundary subject to these conditions is much less than the total groove perimeter, the error introduced by this assumption is considered negligible. 
The non-dimensionalization of important parameters with the use of Eq.(3.10) and Eq.(3.11) is stated in Eqs.(3.21-3.23).

$$
\begin{aligned}
P^{*} & =\frac{P}{L_{g}} \\
A^{*} & =\frac{A}{L_{g}{ }^{2}} \\
\bar{u}^{*} & =-\frac{\bar{u}}{\frac{L_{g}{ }^{2}}{\mu_{l}} \frac{d P_{l}}{d z}}
\end{aligned}
$$

The governing equation can also be rewritten in non-dimensional form by combining Eqs.(3.10, 3.11, 3.16, 3.23) to form Eq.(3.24).

$$
\frac{\partial^{2} u^{*}}{\partial x^{* 2}}+\frac{\partial^{2} u^{*}}{\partial y^{* 2}}=-1
$$

The Poiseuille number can now be obtained using the average velocity from the partial differential equation solution to Eq.(3.24) as seen in Eq.(3.25).

$$
P o_{l}=\frac{8}{\bar{u}^{*}}\left(\frac{A^{*}}{P^{*}}\right)^{2}
$$

One can see from Eq.(3.25) that the area to perimeter ratio plays an important role in determining the Poiseuille number. The variation in the area to perimeter ratio with attachment point, $x^{*}$ and contact angle, $\theta$ is seen in Figure 3.10. The variation of the interfacial boundary length with $x^{*}$ and $\theta$ is seen in Figure 3.11.

The trends in Figure 3.10 will be helpful in distinguishing effects on the Poiseuille number due to shape and average velocity. Interface length variation seen in Figure 3.11 will be important when determining the effects of interfacial shear given that a longer interface with the same shear value will produce an overall greater resistance. 


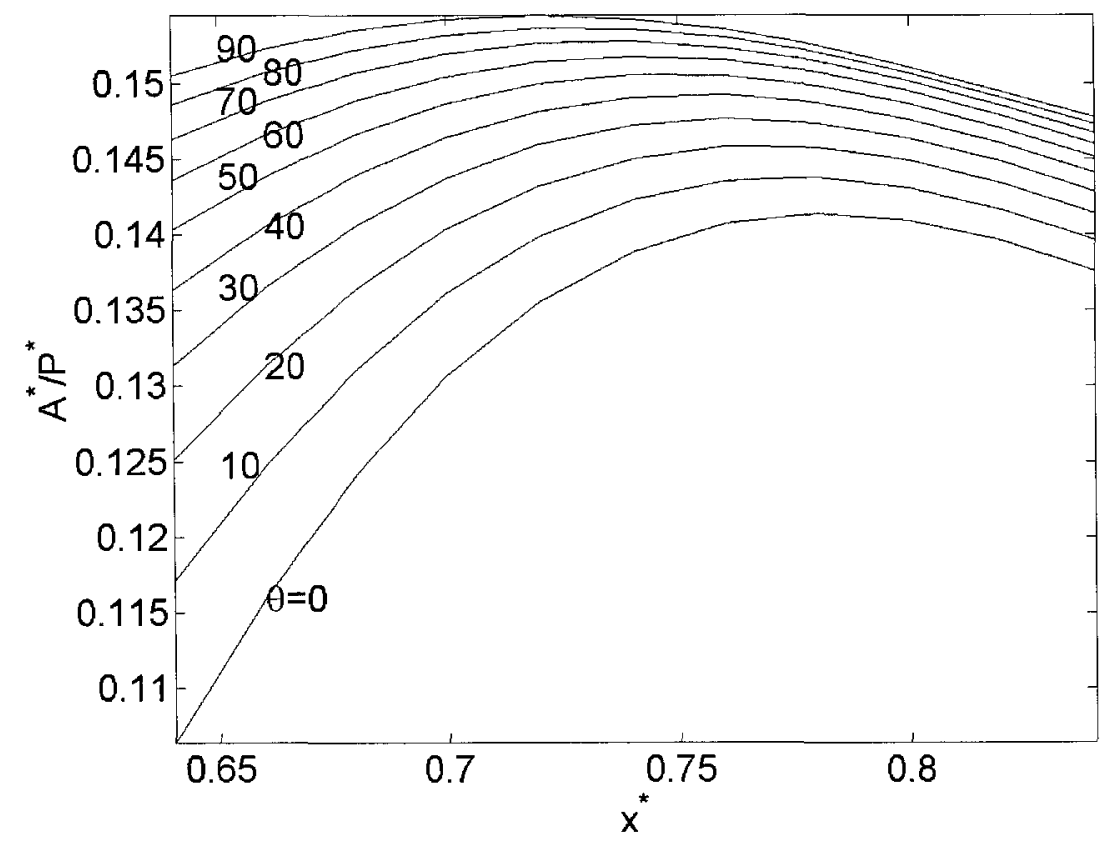

Figure 3.10: Area to Perimeter Variation

\subsubsection{Boundary Conditions}

The liquid groove has three different boundary conditions illustrated in Figure 3.12. The symmetry condition from A-B is stated in Eq.(3.26) as:

$$
\left.\frac{\partial u^{*}}{\partial y^{*}}\right|_{y^{*}=0}=0
$$

The liquid-vapour interface B-C was set either to no slip or having a constant normal shear of magnitude determined from an assumed vapour flow to be discussed later. The solid-liquid interface C-A was set to no slip without exception. 


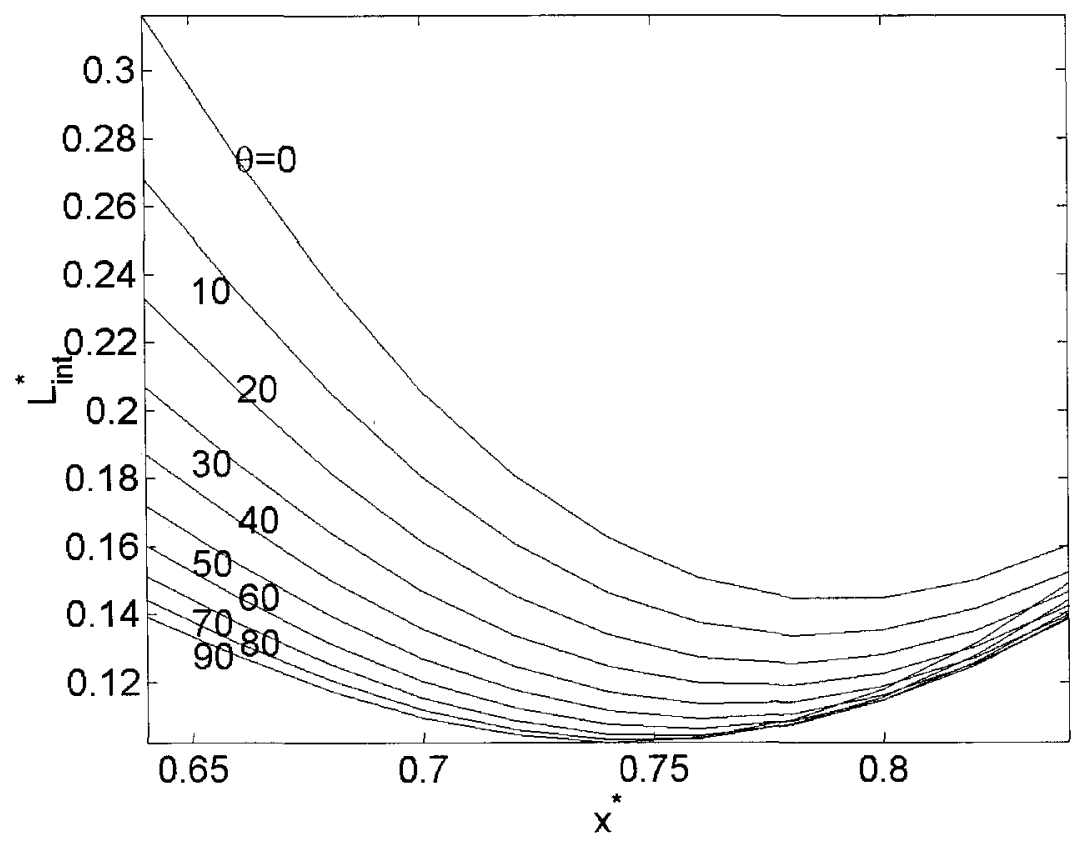

Figure 3.11: Interface Length Variation

\section{Velocity at Liquid-Vapour Interface}

A broader coupled solution between the liquid and vapour can yield the interfacial shear stress and corresponding velocity gradient for a Newtonian fluid. The liquid axial velocity at the liquid-vapour interface is dependent upon the vapour flow. Mass conservation expressed through Eq.(3.27),

$$
\begin{aligned}
\dot{m}_{v} & =\dot{m}_{l} \\
\rho_{v} A_{v} \bar{u}_{v} & =n \rho_{l} A_{l} \bar{u}_{l} \\
\bar{u}_{v} & =n \frac{\rho_{l} A_{l}}{\rho_{v} A_{v}} \bar{u}_{l}
\end{aligned}
$$

requiring the combined mass flow of the circumferential grooves be equal to the counter mass flow of the vapour groove for a given cross-section under steady-state. 


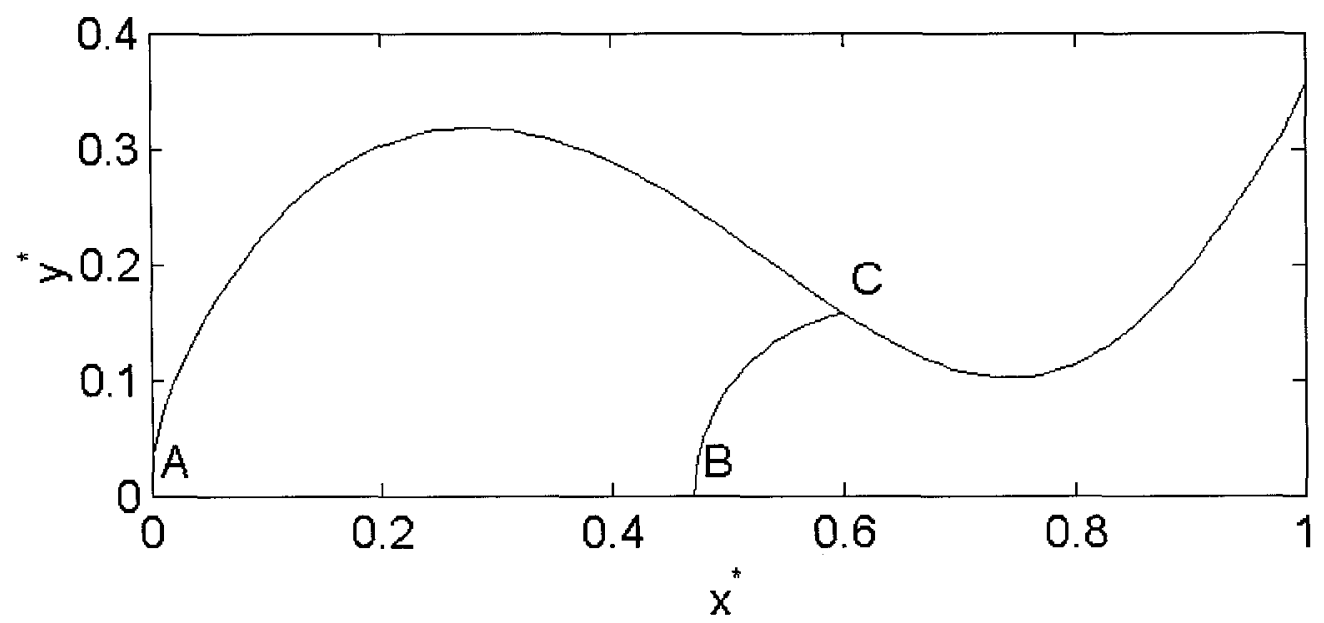

Figure 3.12: Liquid Groove Boundary Segments

Assuming a parabolic vapour velocity profile in Eq.(3.28) and differentiating with respect to the radial vapour direction in Eq.(3.29) gives:

$$
\begin{gathered}
u_{v}=2 \bar{u}_{v}\left[1-\left(\frac{r_{v}}{R_{v}}\right)^{2}\right] \\
\left.\frac{\partial u_{v}}{\partial r_{v}}\right|_{r_{v}=R_{v}}=\frac{-4 \bar{u}_{v}}{R_{v}}
\end{gathered}
$$

The parabolic profile chosen assumes zero velocity at the boundary. This assumption is reasonable because liquid velocity is small and acts almost as a wall.

Shear on the liquid is assumed equal to shear on the vapour at the interface to preclude tearing and is expressed in Eq.(3.30).

$$
\mu_{v} \frac{\partial u_{v}}{\partial x}=\mu_{l} \frac{\partial u_{l}}{\partial x}
$$


Given that the assumed circular vapour groove radial direction is approximately parallel to the liquid-vapour interface normal, one can combine Eqs.(3.27, 3.29, 3.30) to obtain the liquid velocity gradient at the liquid-vapour interface as seen in Eq.(3.31).

$$
\left.\frac{\partial u_{l}}{\partial \hat{n}}\right|_{\mathrm{lv}}=\frac{-4 n \mu_{v}}{R_{v} \mu_{l}}\left(\frac{\rho_{l} A_{l}}{\rho_{v} A_{v}} \overline{u_{l}}\right)
$$

One can see from Eq.(3.31) that the average velocity is needed to get the velocity gradient at the interface and an iterative solution is needed to solve this problem.

\subsection{Solving the Governing Equation}

Two approaches were taken to solve the governing equation. A finite difference method code was written in $\mathrm{C}++$ and Matlab's FEM solver were used. The FEM solver was eventually used exclusively to develop the capillary limit model.

\subsubsection{Finite Difference Model}

In order to solve Eq.(3.24), the finite difference formulation of Eq.(3.32) is used as a starting point to get the Point Gauss-Seidel iteration general formula Eq.(3.33), Ref.[24] with $\zeta=\frac{\Delta x^{*}}{\Delta y^{*}}$.

$$
\begin{aligned}
& \frac{u_{i+1, j}^{*}-2 u_{i, j}^{*}+u_{i-1, j}^{*}}{\left(\Delta x^{*}\right)^{2}}+\frac{u_{i, j+1}^{*}-2 u_{i, j}^{*}+u_{i, j-1}^{*}}{\left(\Delta y^{*}\right)^{2}}=-1 \\
& u_{i, j}^{* k+1}=\frac{1}{2\left(1+\zeta^{2}\right)}\left[\left(\Delta x^{*}\right)^{2}+{u_{i+1, j}^{*}}^{k}+{u_{i-1, j}^{*}}^{k+1}+\zeta^{2}\left({u_{i, j+1}^{*}}^{k}+u_{i, j-1}^{*}{ }^{k+1}\right)\right]
\end{aligned}
$$


Dividing Eq.(3.33) by $\left(\Delta x^{*}\right)^{2}$ and taking the $*$ to represent the new non-dimensional velocity yields Eq.(3.34).

$$
u_{i, j}^{* k+1}=\frac{1}{2\left(1+\zeta^{2}\right)}\left[-1+u_{i+1, j}^{*}{ }^{k}+u_{i-1, j}^{*}{ }^{k+1}+\zeta^{2}\left(u_{i, j+1}^{*}{ }^{k}+u_{i, j-1}^{*}{ }^{k+1}\right)\right]
$$

The shear stress normal to the liquid-vapour interface is known from the simultaneous evaluation of the vapour counterflow. The shear stress can be broken down into normal components with the origin located at the local point where shear stress is being evaluated and with axes parallel to those of the corresponding groove grid. The angle $\xi$ that the normal makes with respect to the $x^{*}$ axis is seen in Eq.(3.35) and Figure 3.13, Ref.[25].

$$
\xi=\arctan \left(-d y^{*} / d x^{*}\right)
$$

The shear stress over viscosity can be written in terms of the interface geometry and change in the flow field based on existing coordinates with Eq.(3.36). Conversion of the unit vector to its trigonometric components is based on the identity of $\cos ^{2}(\xi)+$ sir of the unit vector to its trigonometric components is based on the identity of $\cos ^{2}(\xi)+$ $\sin ^{2}(\xi)=1$

$$
\begin{aligned}
\left(\frac{\tau_{\mathrm{lv}}}{\mu_{l}}\right)^{*} & =\hat{n} \cdot \nabla u^{*} \\
& =\left(n_{\hat{i}}, n_{\hat{j}}\right) \cdot\left(\frac{\partial u^{*}}{\partial x^{*}}, \frac{\partial u^{*}}{\partial y^{*}}\right) \\
& =\left[\cos (\xi) \frac{\partial u^{*}}{\partial x^{*}}+\sin (\xi) \frac{\partial u^{*}}{\partial y^{*}}\right]
\end{aligned}
$$




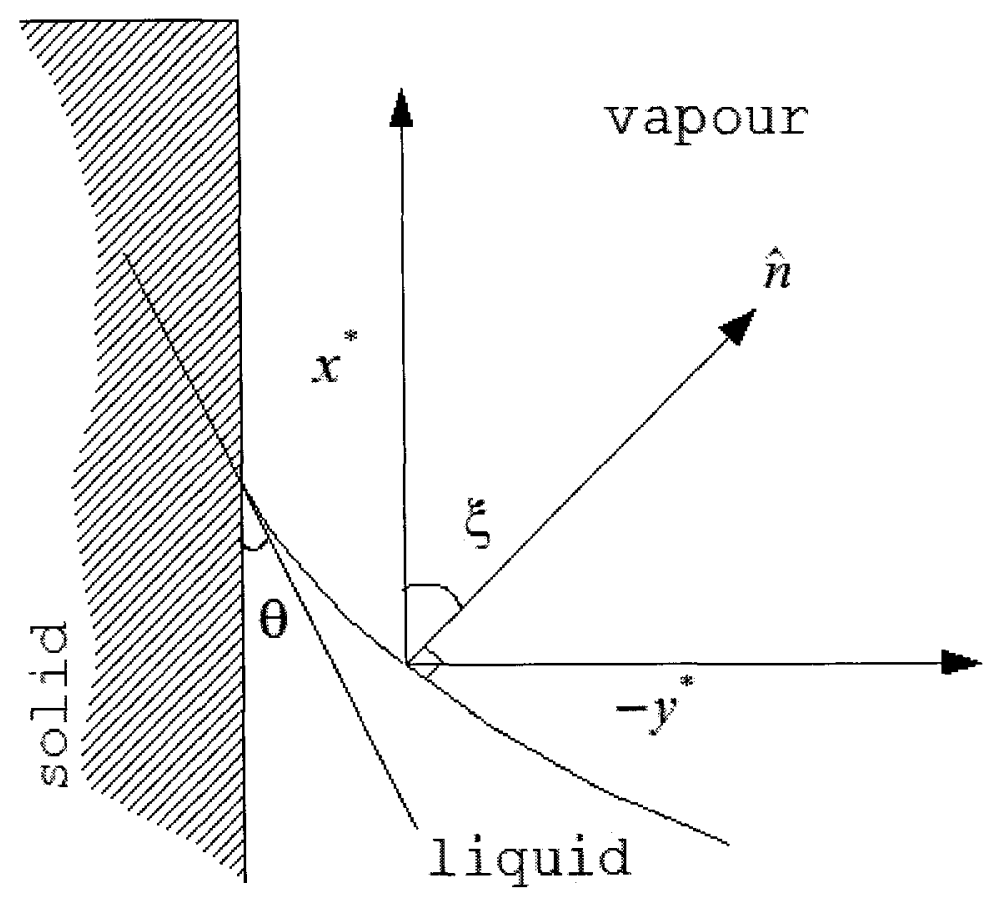

Figure 3.13: Liquid-Vapour Interface Coordinates

A first order backward difference discretization and non-dimensionalization of Eq.(3.31) to obtain the liquid velocity at the liquid-vapour interface is performed in Eq.(3.37).

$$
u_{i, j}^{*}=\frac{-4 n \Delta x^{*} \mu_{v}}{R_{v}^{*} \mu_{l}}\left(\frac{\rho_{l} A_{l}}{\rho_{v} A_{v}} \bar{u}_{l}^{*}\right)+u_{i-1, j}^{*}
$$

Incorporation of the boundary condition at the liquid-vapour interface requires knowing the shape of the meniscus and the velocity gradient in the liquid groove as seen in Figure 3.13. 
Satisfaction of Eq.(3.36) which imposes the shear stress at the interface is achieved by applying a backward-difference first order discretization scheme to the $\frac{\partial u^{*}}{\partial x^{*}}$ component and a forward-difference first order scheme to the $\frac{\partial u^{*}}{\partial y^{*}}$ component. This combination of different schemes is mandatory if placing the origin at the intersection of the axis of symmetry and the groove wall as is seen in Figure 3.14.

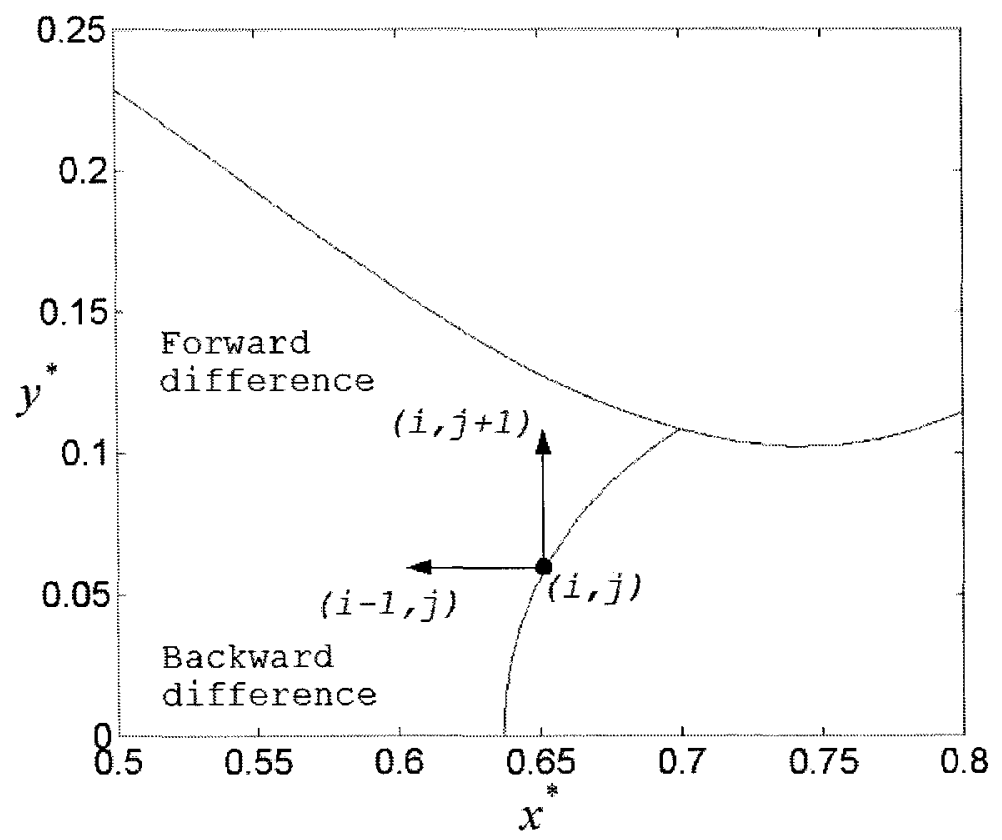

Figure 3.14: Mandatory Discretization Scheme

Isolating Eq.(3.36) for velocity gives Eq.(3.38).

$$
u_{i, j}^{*}=\frac{\left(\frac{\tau_{\mathrm{V}}}{\mu_{l}}\right)^{*}+\frac{\cos (\xi)}{\Delta x^{*}} u_{i-1, j}^{*}+\frac{\sin (\xi)}{\Delta y^{*}} u_{i, j-1}^{*}}{\frac{\cos (\xi)}{\Delta x^{*}}-\frac{\sin (\xi)}{\Delta y^{*}}}
$$

Calculating the interface velocity using the above method requires knowing the liquid mass flow rate. Since the mass flow rate is not known a priori, an iterative approach must be taken. One can first assume a mass flow rate without vapour's influence 
on the solution for the first iteration. The obtained mass flow rate can be used to find the corresponding vapour velocity field to satisfy mass conservation. Successive iterations can yield a more accurate interface velocity profile that tie the physics of the vapour counterflow to the liquid flow until convergence is reached.

There is an inherent numerical instability in Eq.(3.38) due to the variation in $\xi$. Given that $\xi$ remains in the first quadrant, the solution may lead to divergence from the denominator, $\frac{\cos (\xi)}{\Delta x^{*}}-\frac{\sin (\xi)}{\Delta y^{*}}$. The step size can be made different for points near the interface such that Eq.(3.38) does not diverge. To avoid divergence, one can also choose one of two alternative origins for the domain such that the discretization scheme is the same in both directions. Scheme similarity based on chosen origin is summarized in Figure 3.15.

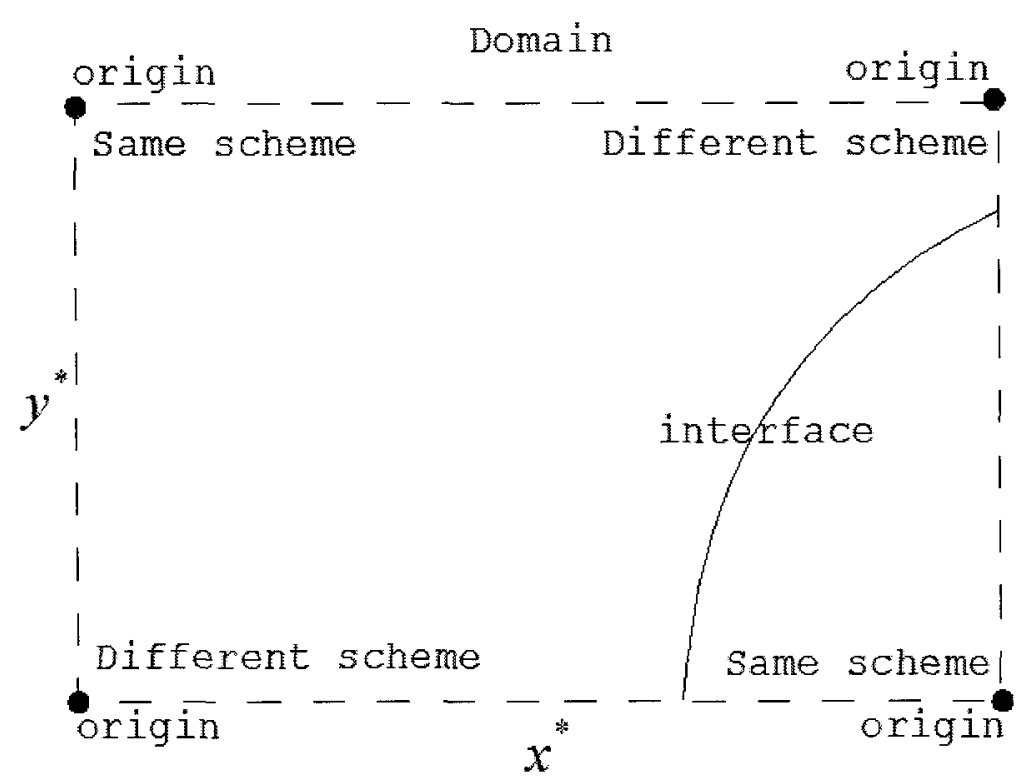

Figure 3.15: Scheme Similarity Based on Domain Origin 


\subsubsection{Finite Element Model}

The groove outline is input to a program utilizing Matlab's Partial Differential Equation Toolbox which generates a mesh given a closed two-dimensional contour. An axial groove contour is uniquely defined by its attachment point and contact angle. Figure 3.16 shows a mesh for a contour having an attachment point at $x^{*}=0.7$ and contact angle at $\theta=45^{\circ}$.

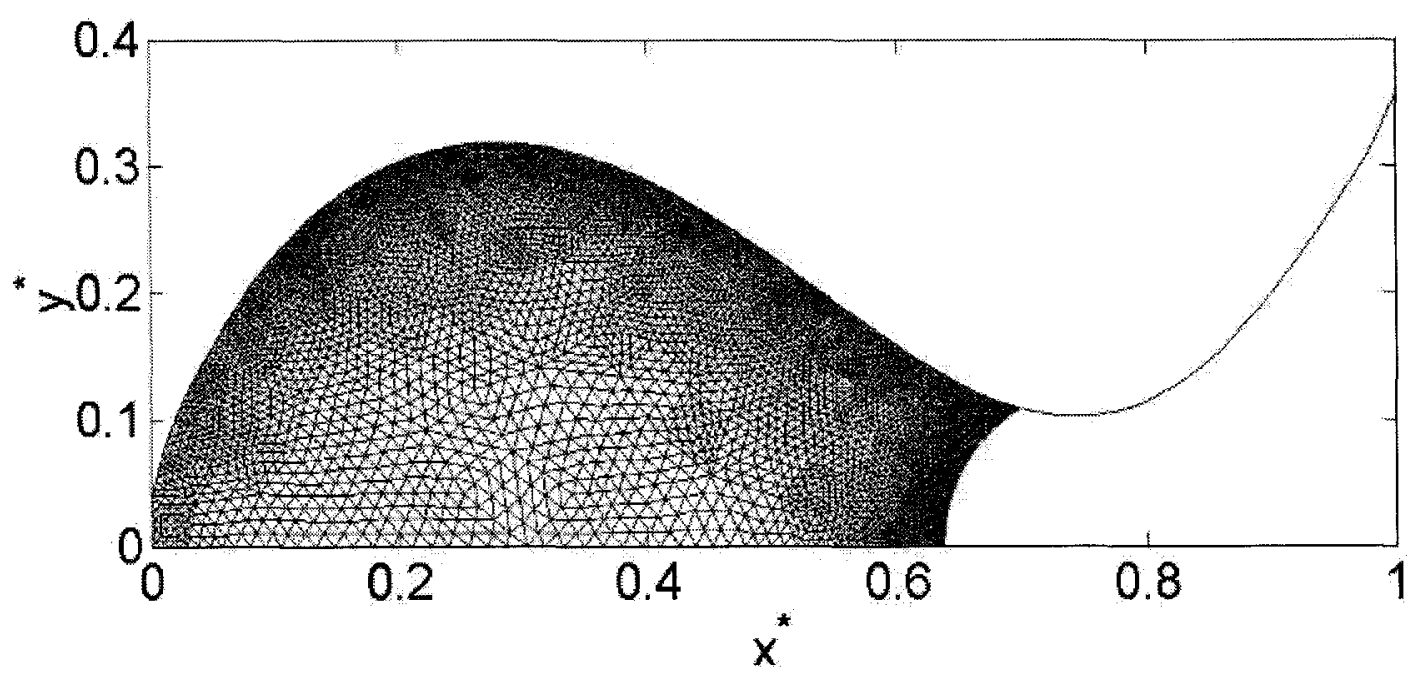

Figure 3.16: Finite Element Mesh, $x^{*}=0.7, \theta=45^{\circ}$

\section{Algorithm for Shear Magnitude at Liquid-Vapour Interface}

The liquid vapour interface was analyzed with no slip and with shear resulting from vapour counterflow. The counterflow shear was assumed constant along the liquidvapour interface and equal to the liquid shear. The shears were set equal to represent no tearing at the interface. An iterative approach was used to find the correct shear to line the liquid-vapour interface. The liquid interfacial shear stress was first set to zero and velocity field was evaluated using the Poiseuille solver. The mass flow rate was obtained by integrating the velocity field. The vapour shear stress at the interface 
was found as a function of the liquid mass flow rate. Numerical experimentation led to the conclusion that liquid and vapour shear variation was nearly linear. This relation is similar to the one made in Ref.[11] pertaining to the linear relationship between interfacial shear and average velocity. It was also found that $\tau_{l}>(1 \rightarrow \infty)$ would give divergent results for $\tau_{v}$ given successive iterations equating the vapour and liquid shear. The chosen liquid-vapour interface shear magnitude was obtained from the linear interpolation of liquid and vapour shears within the input domain of $\left[\tau_{l}^{*}=0, \tau_{l}^{*}=1\right]$. This process is summarized in Figure 3.17 .

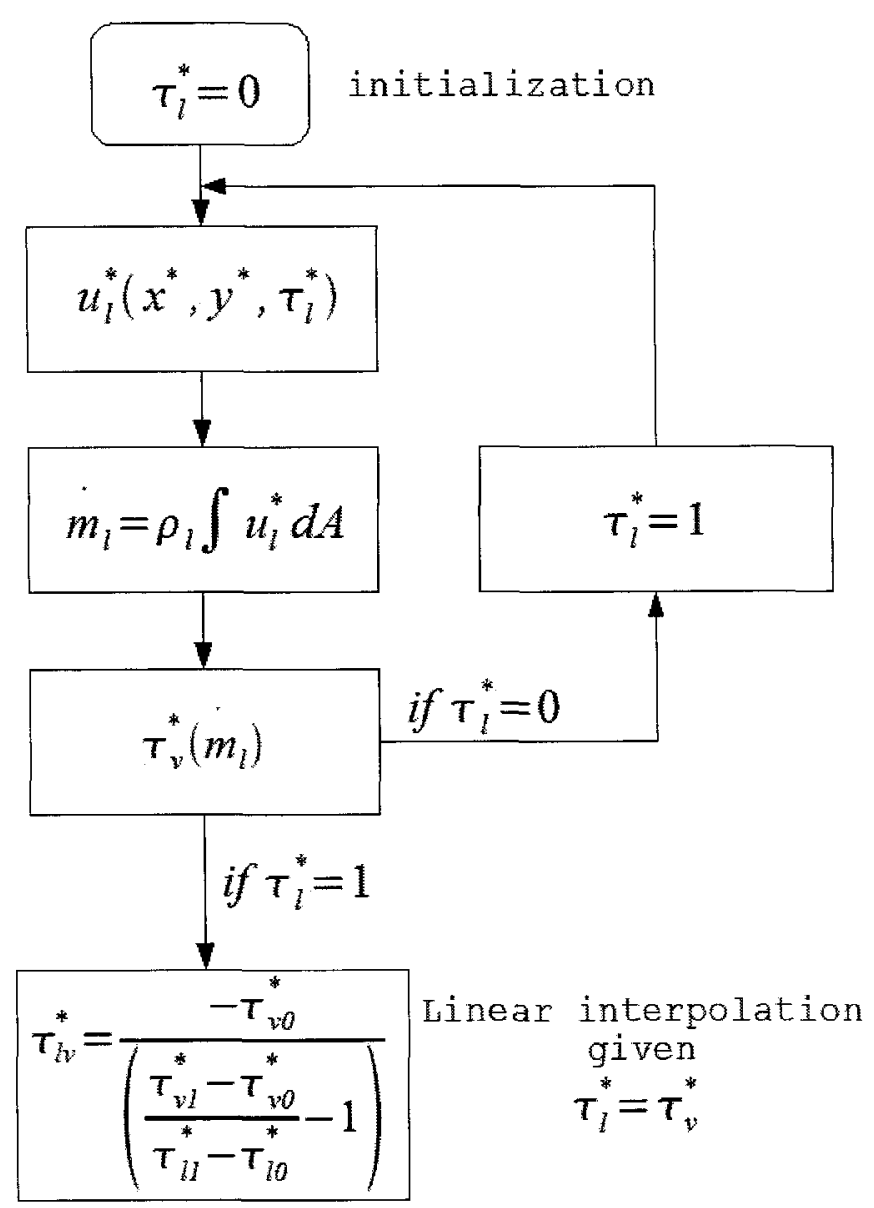

Figure 3.17: Determining Interfacial Shear 


\section{FEM Solver}

Due to the convergence problem associated with a curved interface using the finite difference code, Matlab's PDE toolbox FEM solver was used. The program structure developed allowed for the automated run of a parametric analysis. The variation in attachment point, contact angle, and liquid-vapour boundary was carried through by calling a single function that would repeat the solution algorithm for all the desired input scenarios. Relevant parameters were sent to a data file for future graphical representation. Information such as the velocity field was discarded after creating sample contour plots and calculating the average velocity.

\subsection{Vapour Flow}

The vapour flow is analyzed differently than the liquid flow due to its lower density and higher velocity. The lower density justifies neglecting body forces resulting from tilting. The higher velocity makes the inertial terms important. The vapour groove cross-section is assumed circular throughout for the sake of simplicity making its Poiseuille number theoretically equal to 16 . The vapour can be modelled using a control volume approach as seen in Figure 3.18.

For a low Reynolds number (laminar) flow, one can assume a parabolic velocity profile, Ref.[15]. From balancing the terms seen in Figure 3.18, the following equation is obtained:

$$
\frac{d P_{v}}{d z}=\underbrace{\frac{-8 \mu_{v} \dot{m}_{v}}{A_{v} r_{h, v}^{2} \rho_{v}}}_{\text {viscous }}-\underbrace{\frac{8 \dot{m}_{v}}{3 A_{v}^{2} \rho_{v}} \frac{d \dot{m}_{v}}{d z}}_{\text {inertial }}
$$




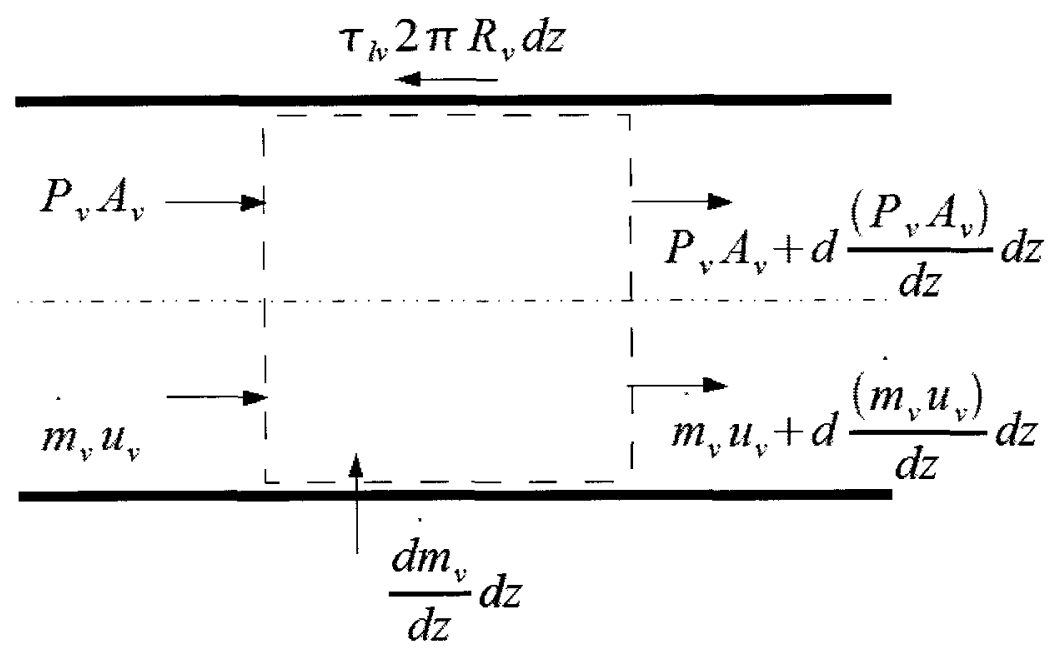

Figure 3.18: Axial Vapour Control Volume

The vapour mass flow rate can be related to the heat transferred and the latent heat of vapourization using:

$$
\dot{m}_{v}=\frac{Q}{h_{f g}}
$$

Combining Eq.(3.39) and Eq.(3.40) results in:

$$
\frac{d P_{v}}{d z}=\frac{-8 \mu_{v} \dot{m}_{v}}{A_{v} R_{v}^{2} \rho_{v} h_{f g}} Q-\frac{4}{3 A_{v}^{2} h_{f g}^{2} \rho_{v}} \frac{d Q^{2}}{d z}
$$

\subsection{Combining Liquid and Vapour Flows}

From the first law of thermodynamics with the assumption that heat transferred through the heat pipe casing is negligible compared to that transferred by liquid convection in the adiabatic portion, the average liquid velocity can be approximated using Eq.(3.42).

$$
\bar{u}_{l}=\frac{Q}{n A_{l} \rho_{l} h_{f g}}
$$


By combining Eqs.(3.8, 3.17, 3.18, and 3.42), one can obtain:

$$
\frac{d P_{l}}{d z}=-\frac{\left(f_{l} R e_{l}\right) \mu_{l}}{2 n A_{l} r_{h l}^{2} h_{f g} \rho_{l}} Q+\left.\frac{d P_{l}}{d z}\right|_{\vec{g}}
$$

The condition for capillary limit avoidance is dependent on the difference between the changes of vapour and liquid pressures along the heat pipe and can be noted as:

$$
\frac{\sigma}{R_{1}} \geq \int_{0}^{L}\left(\frac{d P_{v}}{d z}-\frac{d P_{l}}{d z}\right) d z
$$

The expansion of Eq.(3.44) using Eq.(3.43) and Eq.(3.41) results in Eq.(3.45).

$$
\frac{\sigma}{R_{1}} \geq \int_{0}^{L}\left(\frac{8 \mu_{v}}{A_{v} R_{v}^{2} \rho_{v} h_{f g}} Q+\frac{4}{3 A_{v}^{2} h_{f g}^{2} \rho_{v}} \frac{d Q^{2}}{d z}+\frac{\left(f_{l} R e_{l}\right) \mu_{l}}{2 n A_{l} r_{h l}^{2} h_{f g} \rho_{l}} Q-\left.\frac{d P_{l}}{d z}\right|_{\vec{g}}\right) d z
$$

Assuming no heat leak at either end; $Q(0)=Q(L)=0$ negates the following term:

$$
\begin{aligned}
\frac{4}{3 A_{v}^{2} h_{f g}^{2} \rho_{v}} \int_{0}^{L} \frac{d Q^{2}}{d z} d z & =\frac{8}{3 A_{v}^{2} h_{g h}^{2} \rho_{v}} \int_{Q(0)}^{Q(L)} Q d Q \\
& =\frac{4}{3 A_{v}^{2} h_{f g}^{2} \rho_{v}}\left(Q^{2}(L)-Q^{2}(0)\right) \\
& =0
\end{aligned}
$$

One can rearrange Eq.(3.45) for the heat transport factor:

$$
\left(Q L_{\mathrm{eff}}\right)_{\max }=\frac{2 h_{f g}\left(\frac{\sigma}{R_{1}}+\left.\Delta P_{l}\right|_{\vec{g}}\right)}{\frac{16 \mu_{v}}{A_{v} R_{v}^{2} \rho_{v}}+\frac{\left(f_{l} R e_{l}\right) \mu_{l}}{n A_{l} r_{h l}^{2} \rho_{l}}}
$$




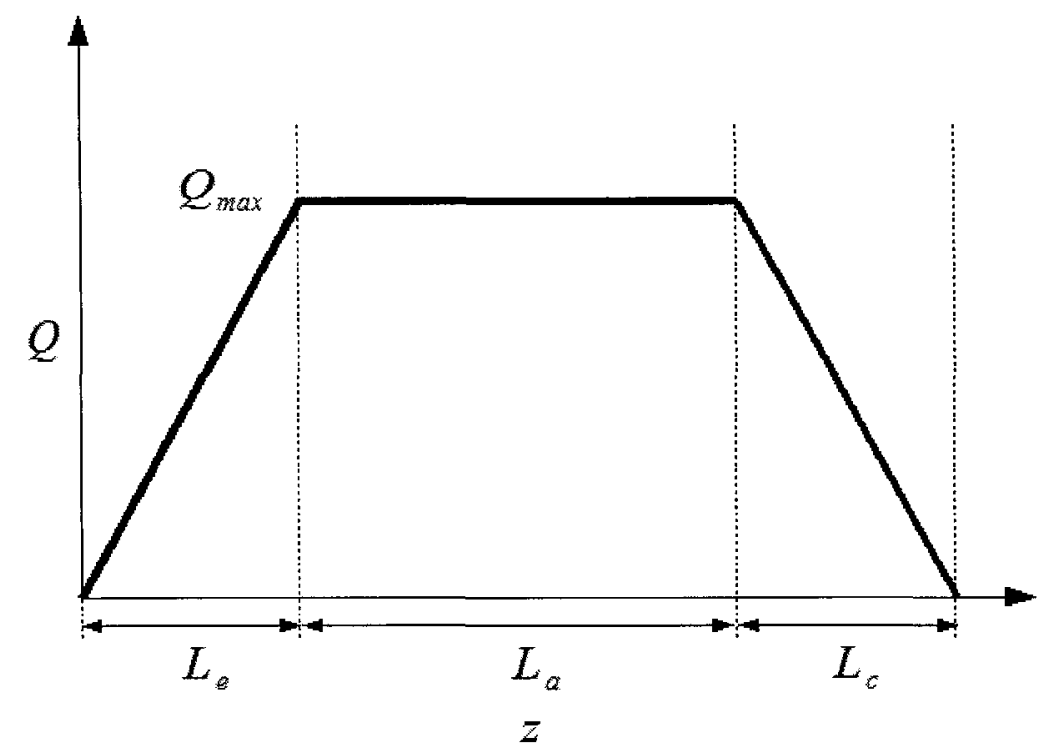

Figure 3.19: Assumed Axial Heat Transfer

In order to solve for the maximum heat transfer at the capillary limit, one introduces an axial heat transfer distribution. The radial heat input and output throughout the evaporator and condenser are assumed constant and there is no heat transfer at the endpoints. This can be seen graphically in Figure 3.19. One can integrate along the heat pipe assuming the above heat distribution to obtain

$$
\begin{aligned}
Q_{\max } L_{\mathrm{eff}} & =\int_{0}^{L} Q d z \\
& =Q_{\max }\left(0.5 L_{c}+L_{a}+0.5 L_{e}\right)
\end{aligned}
$$

Combining Eq.(3.19), Eq.(3.46), and Eq.(3.47) leads to the final equation for the maximum heat transfer at the capillary limit:

$$
Q_{\text {max }}=\frac{2 h_{f g}\left(\frac{\sigma}{R_{1}}+\left.\Delta P_{l}\right|_{\vec{g}}\right)}{\left(\frac{16 \mu_{v}}{A_{v} R_{v}^{2} \rho_{v}}+\frac{P o_{l} \mu_{l}}{n A_{l} r_{h l}^{2} \rho_{l}}\right)\left(0.5 L_{c}+L_{a}+0.5 L_{e}\right)}
$$




\subsection{Capillary Limit Code Structure}

The process used to obtain the capillary limit for a given contact angle, attachment point, and test temperature is shown in Figure 3.20. The rounded rectangles represent user input and the diamond represents the conditional necessity to introduce heat pipe geometry and ammonia properties when solving for the Poiseuille number if interfacial vapour counterflow is to be modelled.

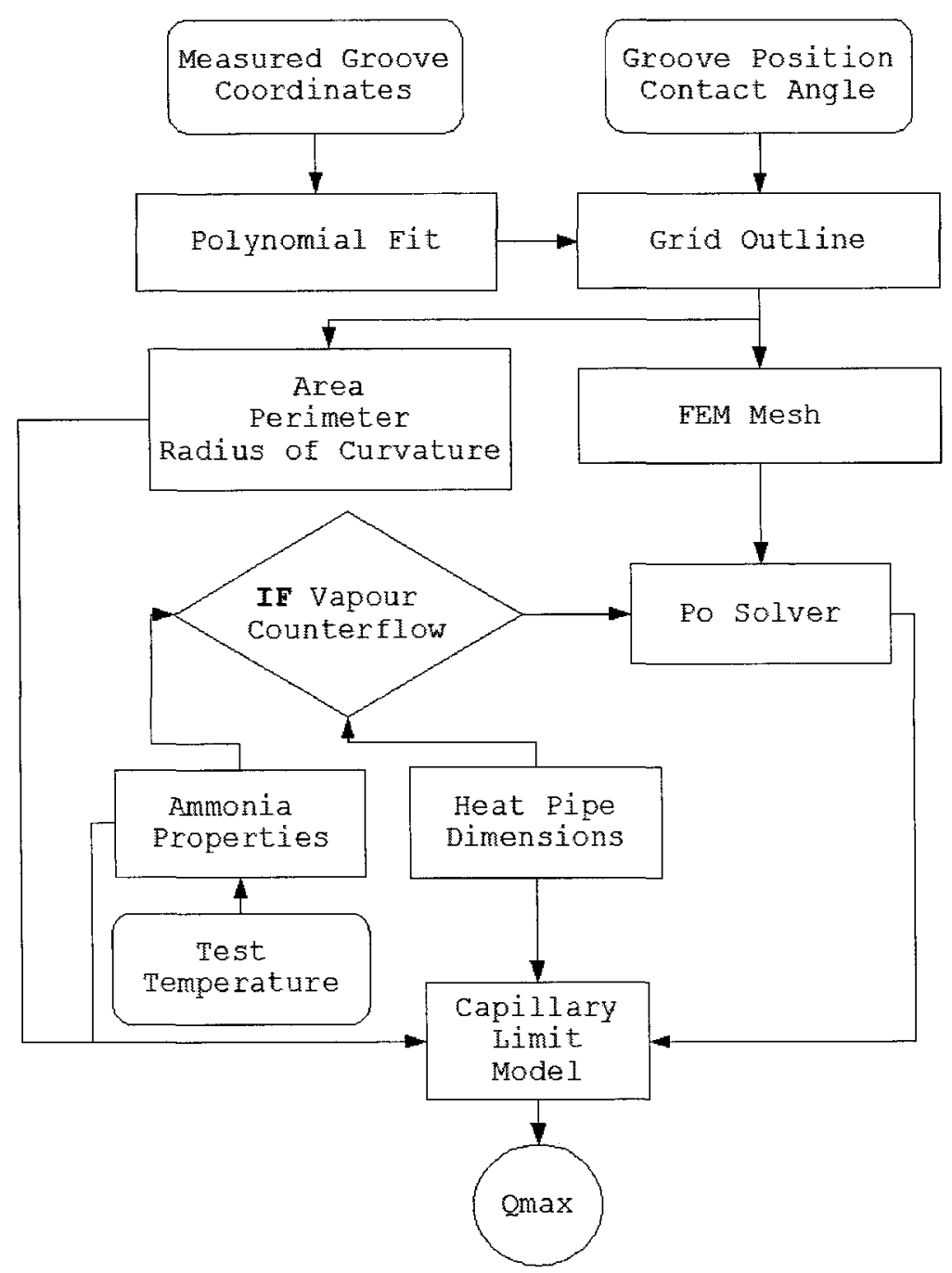

Figure 3.20: Capillary Limit Algorithm Flowchart 


\subsection{Numerical Results}

The finite difference model gave satisfactory results when compared to a working FEM code except for when shear was set normal to a curved liquid-vapour interface. Problems arose for this condition and rather than persisting with trying to make the condition work, Matlab's PDE toolbox was used since numerically solving Poison's equation was only an intermediate step and the overall model was considered more important.

The variation in contact angle and attachment point was restricted from $0^{\circ}$ to $90^{\circ}$ and 0.64 to 0.84 , respectively. Physically speaking, the limiting radius of curvature is infinite for there to be a necessary pressure difference between the liquid and vapour. Cases not showing the necessary pressure difference are shown nonetheless because they are mathematically feasible. The groove neck is found at $x^{*}=0.74$ and a spread of $\Delta x^{*}=0.1$ in each direction was considered reasonable. The exact position of the attachment point will vary according to the axial position, heat applied, and the amount of fluid charged. 


\subsubsection{Velocity Fields Resulting from Different Boundary Conditions}

Velocity contours are shown with $\theta=0^{\circ}$ for no slip at $x^{*}=0.64$, no shear at $x^{*}=0.74$, and vapour counterflow at $x^{*}=0.84$ in Figure 3.21, Figure 3.22, and Figure 3.23, respectively.

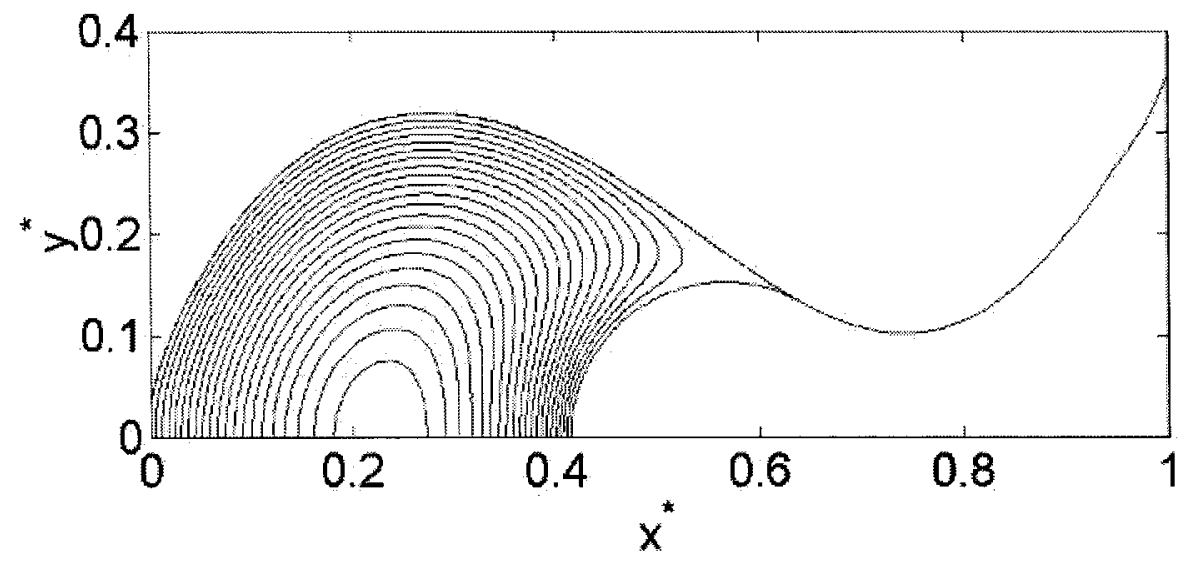

Figure 3.21: No Slip Velocity Contour, $x^{*}=0.64, \theta=0^{\circ}$

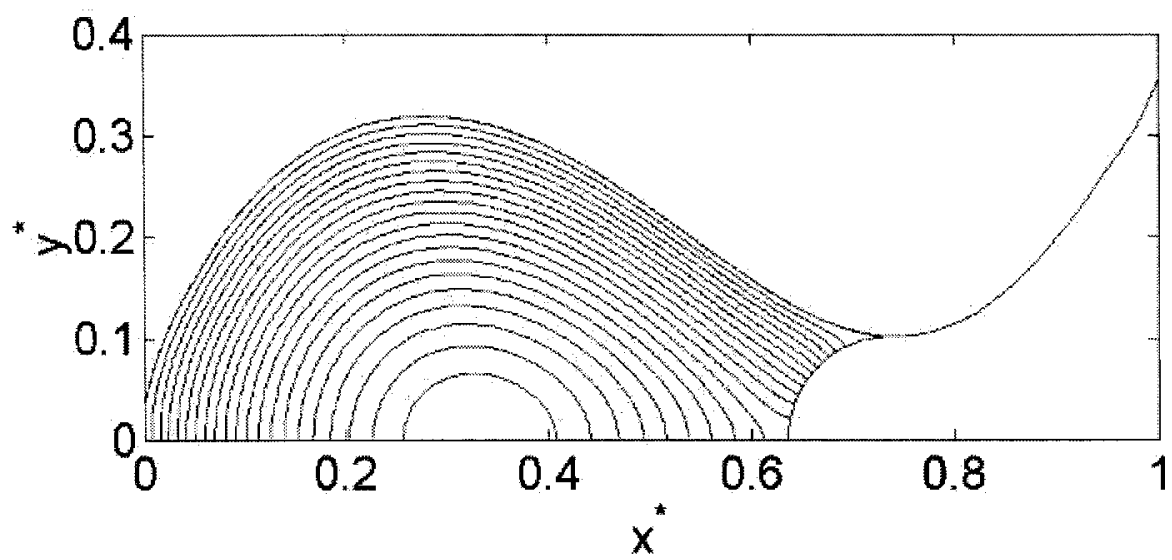

Figure 3.22: No Shear Velocity Contour, $x^{*}=0.74, \theta=0^{\circ}$ 


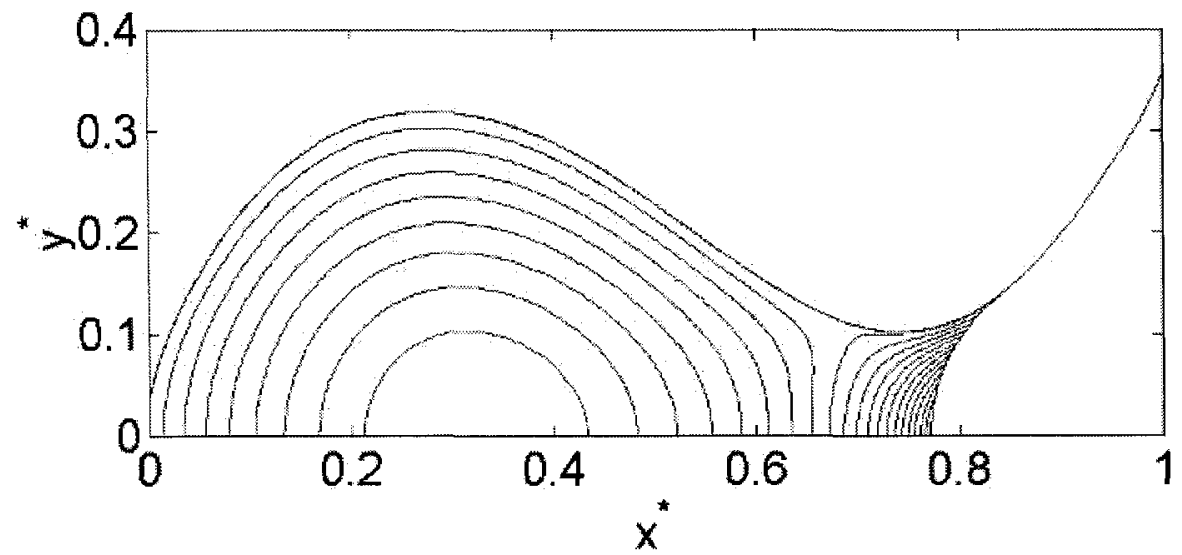

Figure 3.23: Vapour Counterflow Shear Velocity Contour, $x^{*}=0.84, \theta=0^{\circ}$

One can check the validity of the boundary conditions imposed by observing Figures 3.21-3.23. All velocity contours crossing the axis of symmetry do so with perpendicularity. The no-slip condition makes adjacent contours in Figure 3.21 nearly parallel, which is to be expected. The no-shear condition in Figure 3.22 is verified when seeing the contours perpendicular to the liquid-vapour interface. The vapourcounterflow condition shown in Figure 3.23 causes two separate contour islands, indicating a change in flow direction. This can be explained by the pulling effect that the vapour travelling in the opposite direction has on the liquid. 


\subsubsection{Contact Angle Variation}

The variation in contact angle was observed at the three attachment points of $x^{*}=$ $\left[0.64,0.74\right.$ (the neck), and 0.84]. This angle variation between $0^{\circ}$ and $90^{\circ}$ can be seen in Figure 3.24.

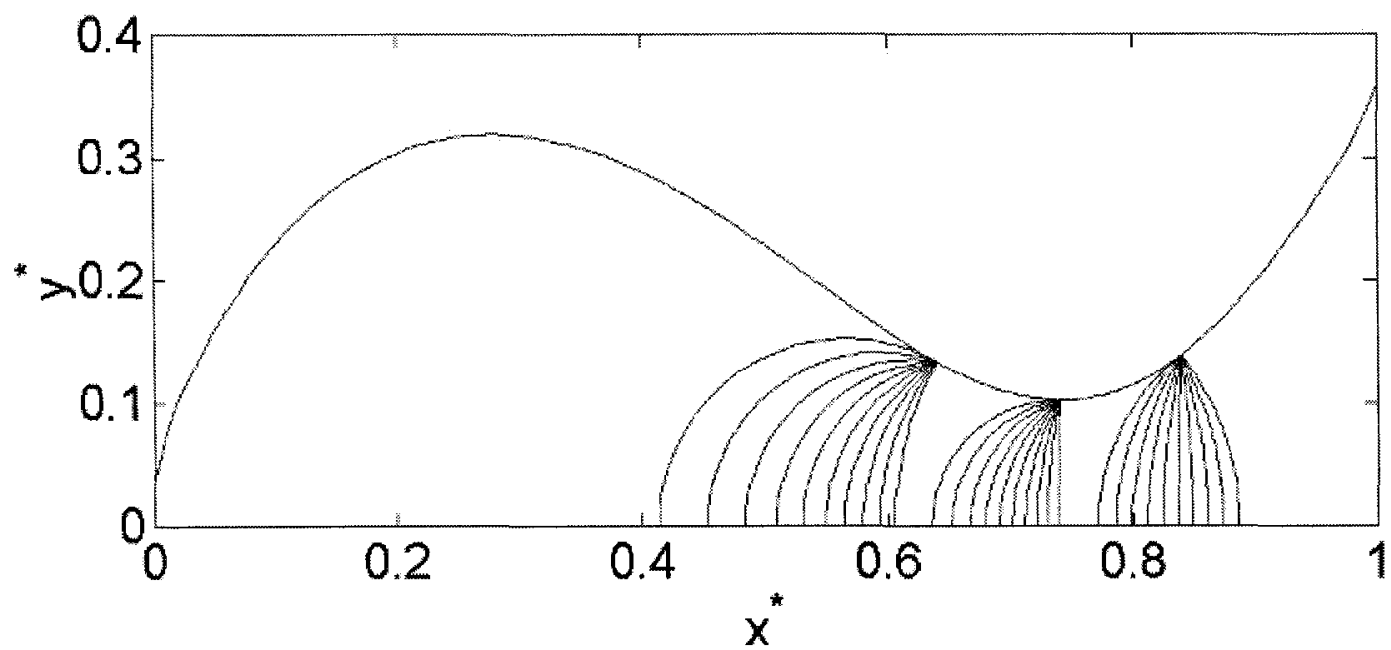

Figure 3.24: Contact Angle Variation at $x^{*}=0.64,0.74$, and 0.84

\section{No Slip}

The variation in Poiseuille number with angle for no slip is shown in Figure 3.25. It is seen from Figure 3.25 that for the same contact angle, the Poiseuille number is greatest at the neck groove. This can be explained by the larger area to perimeter ratio around the neck seen in Figure 3.10 and relating it to Eq.(3.25).

Two important factors playing a crucial role in determining the capillary limit are the cross-sectional area of the liquid in the groove and the interfacial radius of curvature. The size of the cross-sectional area is related to how much fluid is flowing within the liquid groove, which can be related to how much heat is carried with 


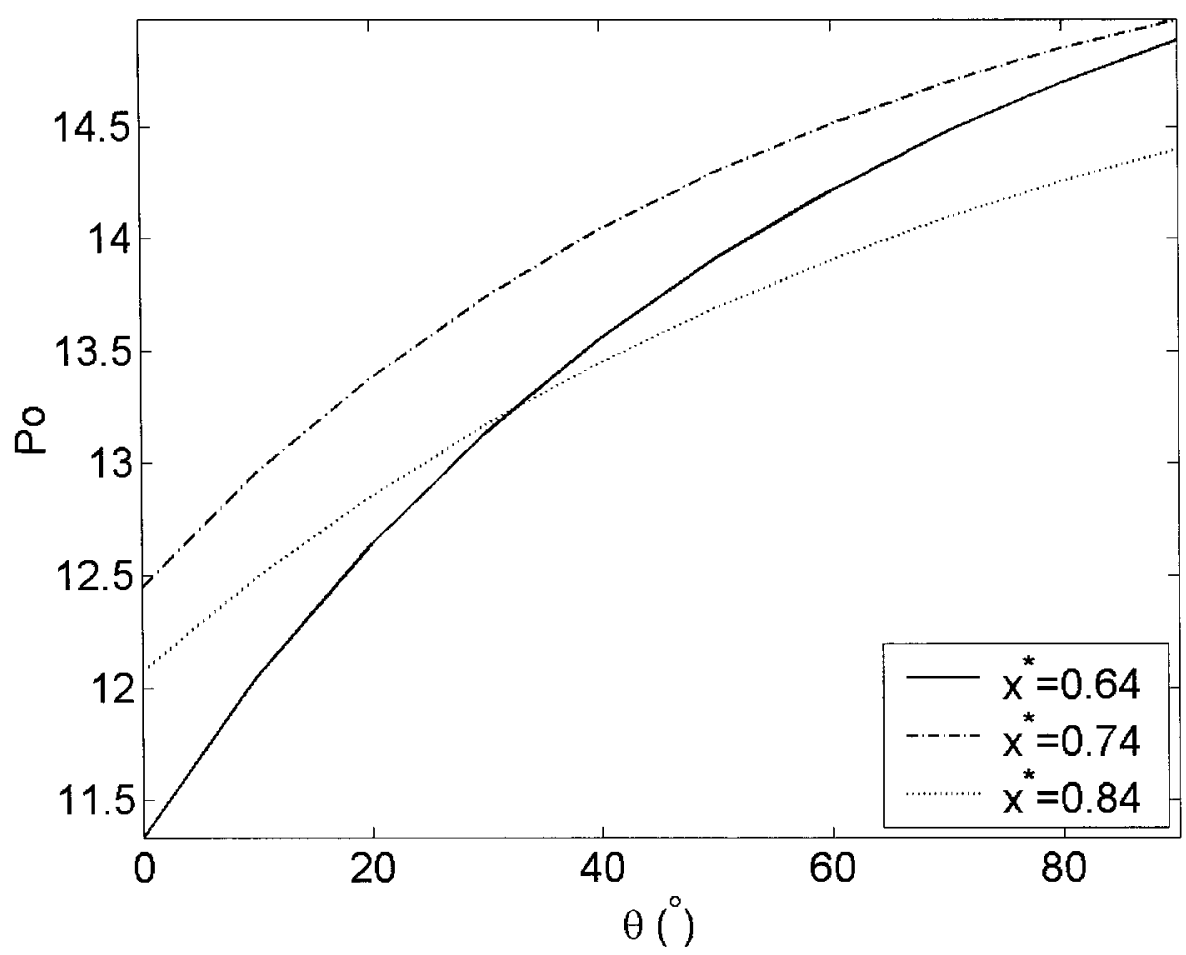

Figure 3.25: Po Variation with Contact Angle, No Slip

the latent heat of vapourization via Eq.(3.40). The interfacial radius of curvature is related to the maximum pressure difference between the vapour and liquid, which is related to the capillary limit via Eq.(3.48).

The variation in capillary limit with contact angle for the three stations shown in Figure 3.24 is seen in Figure 3.26. One can see in Figure 3.26 that the highest heat transfer occurs at $\theta=0^{\circ}$ for $x^{*}=0.74$ and 0.84 . This is because $\theta=0^{\circ}$ allows for the smallest radius of curvature and corresponding highest capillary pressure. It is interesting to note that the highest heat transfer occurs at $\theta=40^{\circ}$ for $x^{*}=0.64$ even though the maximum capillary pressure can be sustained at $\theta=0^{\circ}$. The area of liquid lost due to the curvature does not allow for as much heat to be transported 


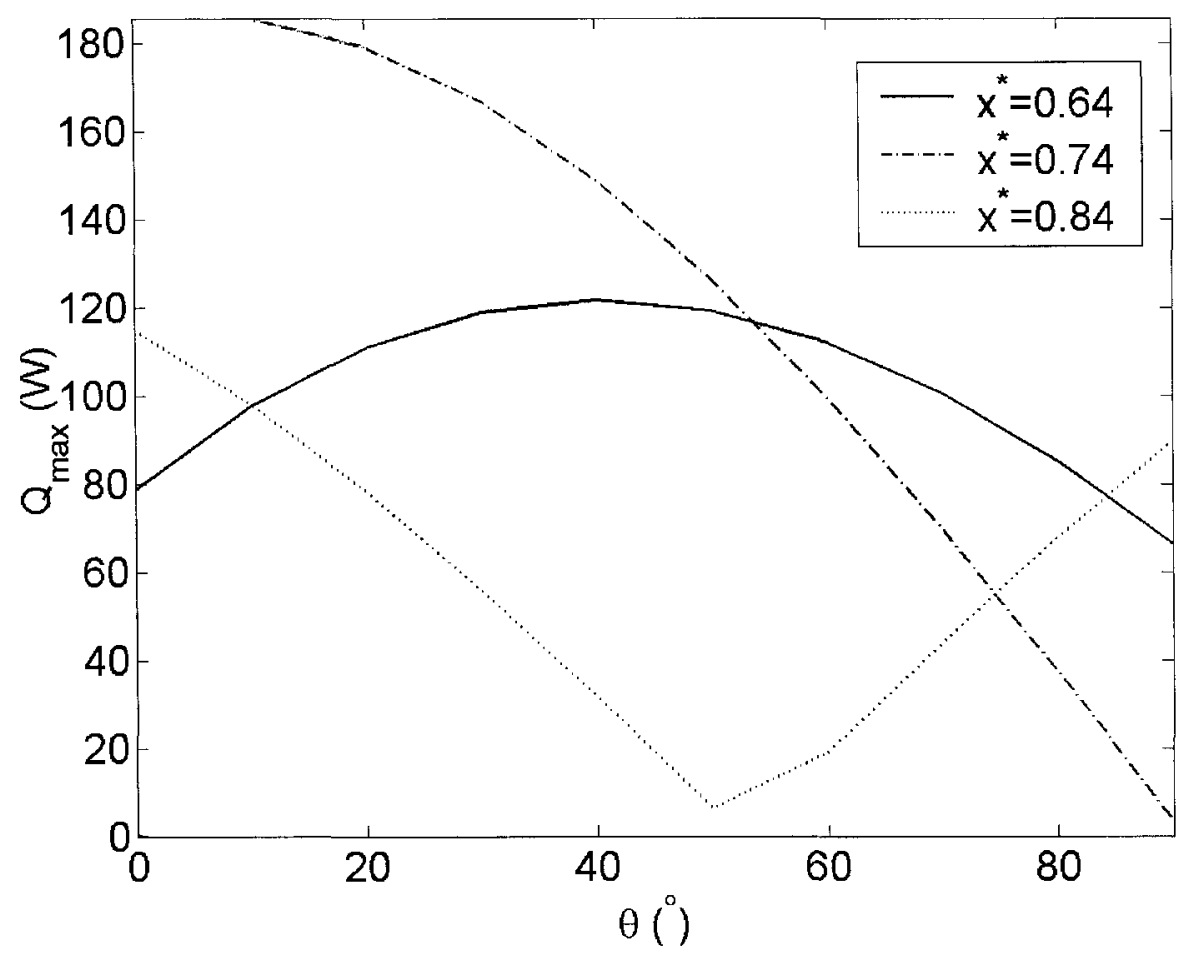

Figure 3.26: Capillary Limit Variation with Contact Angle, No Slip

as in the case of $\theta=40^{\circ}$ with a lower capillary pressure. The cases of $\theta \geq 50^{\circ}$ for station $x^{*}=0.84$ are ignored since they represent a convex curvature and could not sustain an ammonia heat pipe cycle. It is to be noted that the maximum contact angle allowable to produce a positive capillary pressure varies with $x^{*}$ based on the groove geometry.

\section{Vapour Counterflow Shear}

The variation in Poiseuille number with contact angle is shown in Figure 3.27. The Poiseuille number transition with $\theta$ is less smooth for the counterflow condition compared to the no-slip condition as seen when comparing Figure 3.25 and Figure 3.27. This is due to the varying magnitude of the interfacial shear stress and the size 


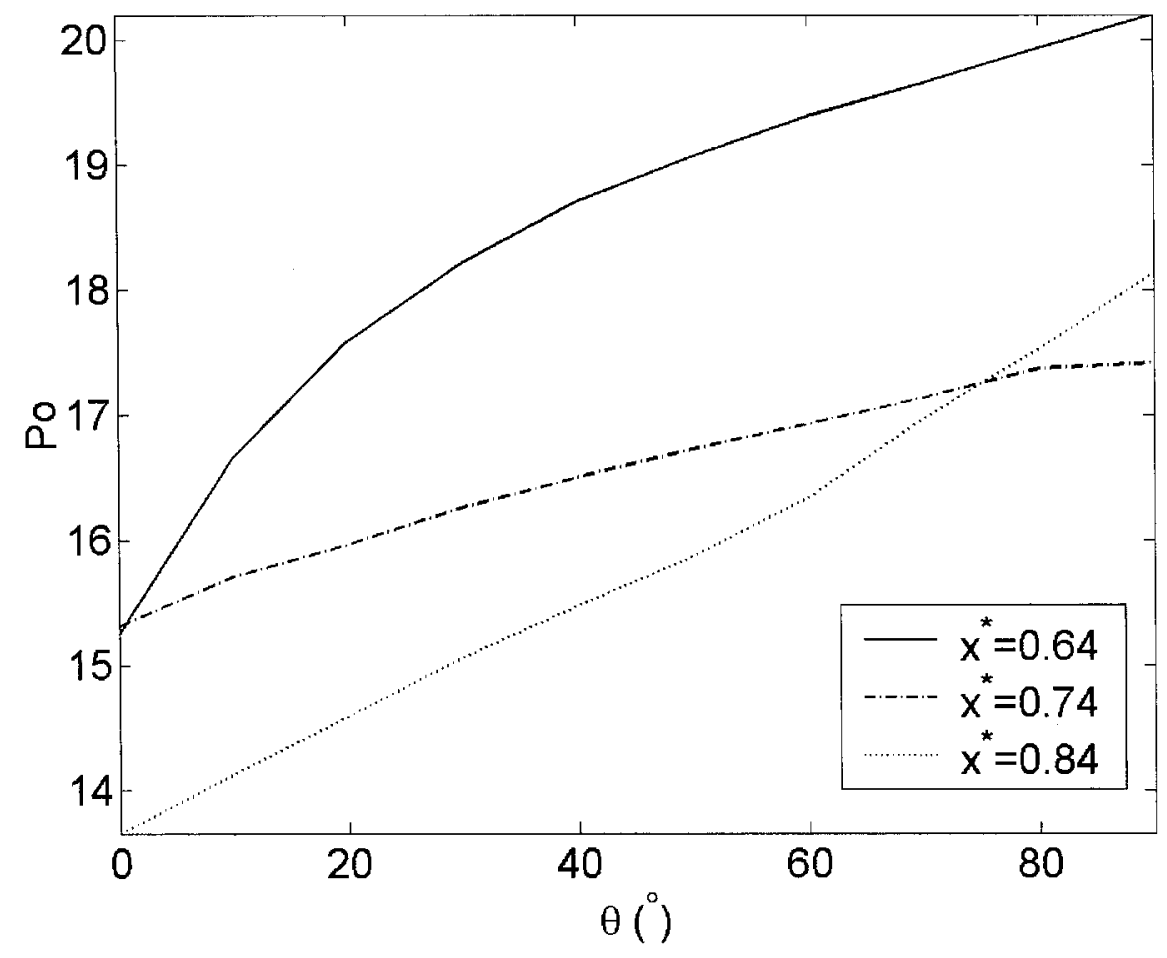

Figure 3.27: Po Variation with Contact Angle, Vapour Counterflow

of the affected interface. The Poiseuille number in Figure 3.27 is highest with $x^{*}=$ 0.64 because the interface there is the largest and so the shear affects it the most. The apparent change in concavity of the $x^{*}=0.84$ curve at $\theta=50^{\circ}$ is due to increase in size of the interface after the concavity of the interface itself changes as shown in Figure 3.24. The heat transfer variation with contact angle can be seen in Figure 3.28. The main difference between the results in Figure 3.26 and Figure 3.28 is that the highest capillary limit for $x^{*}=0.74$ occurs at $\theta=0^{\circ}$ with no slip and $\theta=10^{\circ}$ with vapour counterflow. The fact that less heat can be transferred at $0^{\circ}$ is due to the larger interface producing a greater shearing effect on the flow. This discrepancy in contact angle and maximum capillary limit will require the examination of both $\theta=0^{\circ}$ and $\theta=10^{\circ}$ as possible parameters to get the maximum capillary limit when 


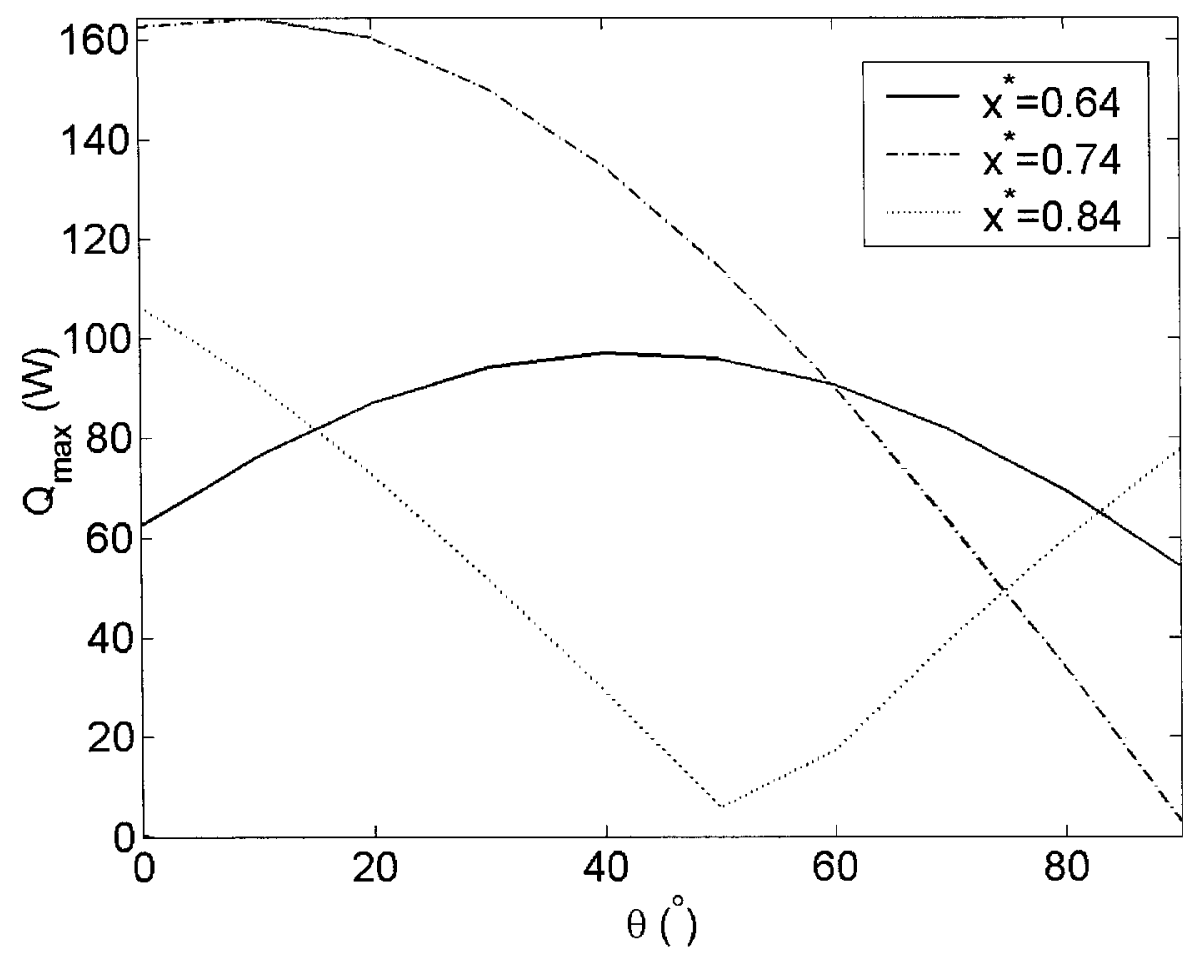

Figure 3.28: Capillary Limit Variation with Contact Angel, Vapour Counterflow varying the attachment point. 


\subsubsection{Attachment Point Variation}

Varying the contact angle revealed that the maximum heat transfer occurs near the groove neck and with $\theta=0^{\circ}$ and $\theta=10^{\circ}$ for no slip and vapour counterflow shear, respectively. The chosen task now is to vary the attachment point between $x^{*}=[0.64,0.84]$ at $\theta=\left[0^{\circ}\right.$ and $\left.10^{\circ}\right]$ in order to find the maximum heat transfer. This will be done for both the no-slip and interfacial shear boundary conditions.

\section{No Slip}

The variation in Poiseuille number with attachment point is seen in Figure 3.29. The shape of the curves in Figure 3.29 are fairly similar and the Poiseuille number

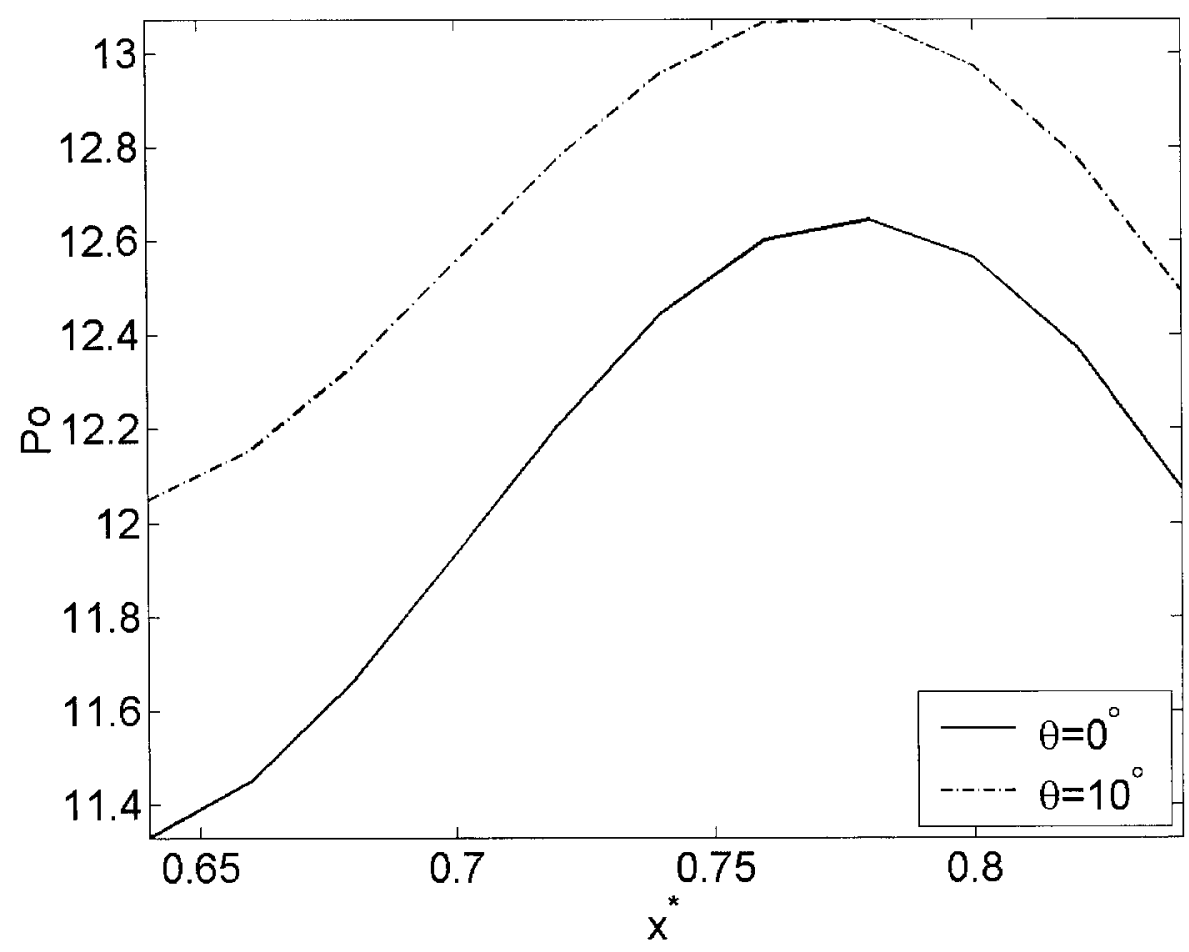

Figure 3.29: Po Variation with Attachment Point, No Slip 
is greater for $\theta=10^{\circ}$. Comparison of this trend with Figure 3.10 indicates that the larger Poiseuille number is most likely caused by the change in area to perimeter ratio since there is very little difference in the average velocity given such a small change in domain.

The variation in capillary limit with $x^{*}$ for $\theta=0^{\circ}$ and $\theta=10^{\circ}$ is seen in Figure 3.30. One can see in Figure 3.30 that the peak heat transfer occurs at $x^{*}=0.76$ for

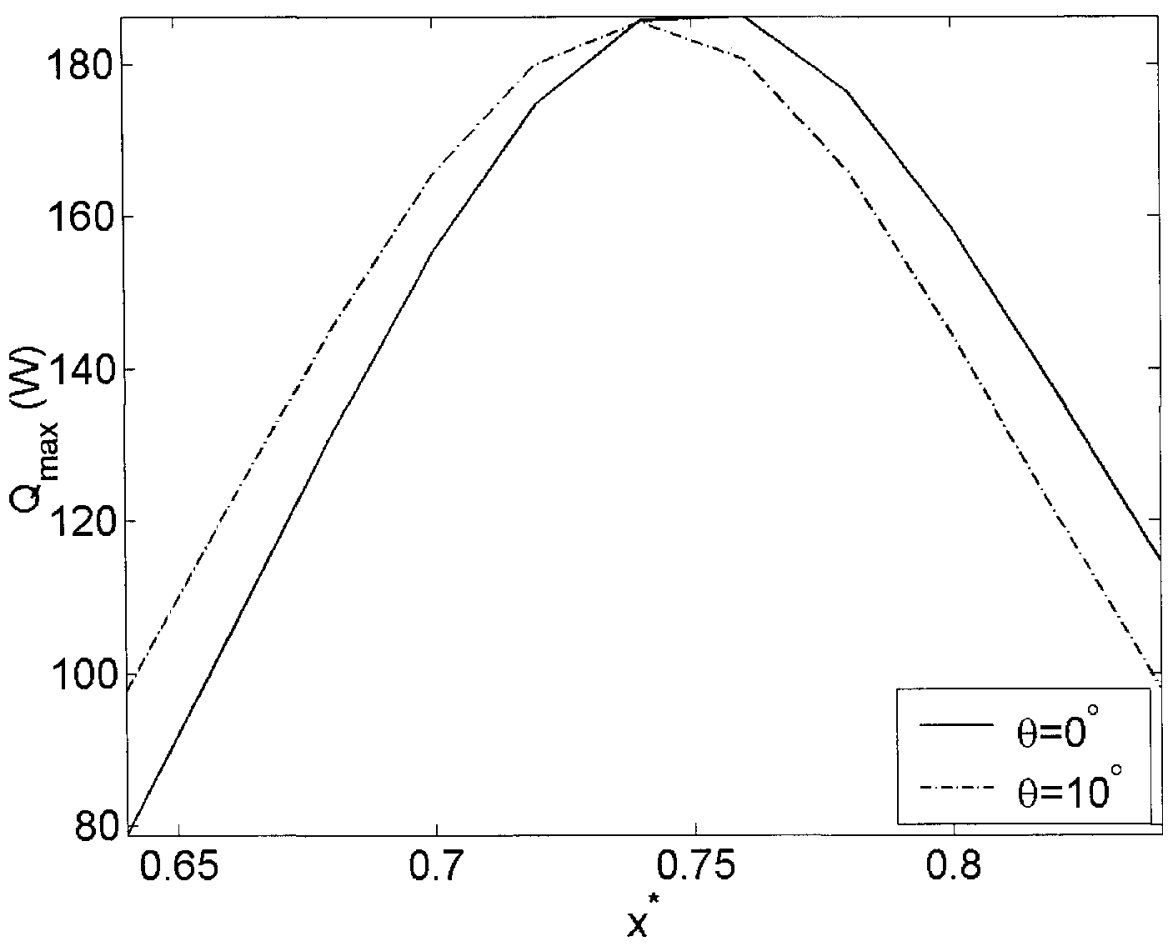

Figure 3.30: Capillary Limit Variation with Attachment Point, No Slip

$\theta=0^{\circ}$ and $x^{*}=0.74$ for $\theta=10^{\circ}$. The case with $\theta=0^{\circ}$ has a slightly higher heat transfer. It is interesting to note that the highest heat transfer does not occur at the groove neck. It occurs on the side where the liquid groove is largest. It is observed that the gain in area and resultant mass flow rate when moving the attachment point 
is more important than having the maximum capillary pressure at the groove neck.

\section{Vapour Counterflow Shear}

The variation in Poiseuille number with attachment point for $\theta=0^{\circ}$ and $\theta=10^{\circ}$ is seen in Figure 3.31. The nearly linear drop in Poiseuille number in Figure 3.31

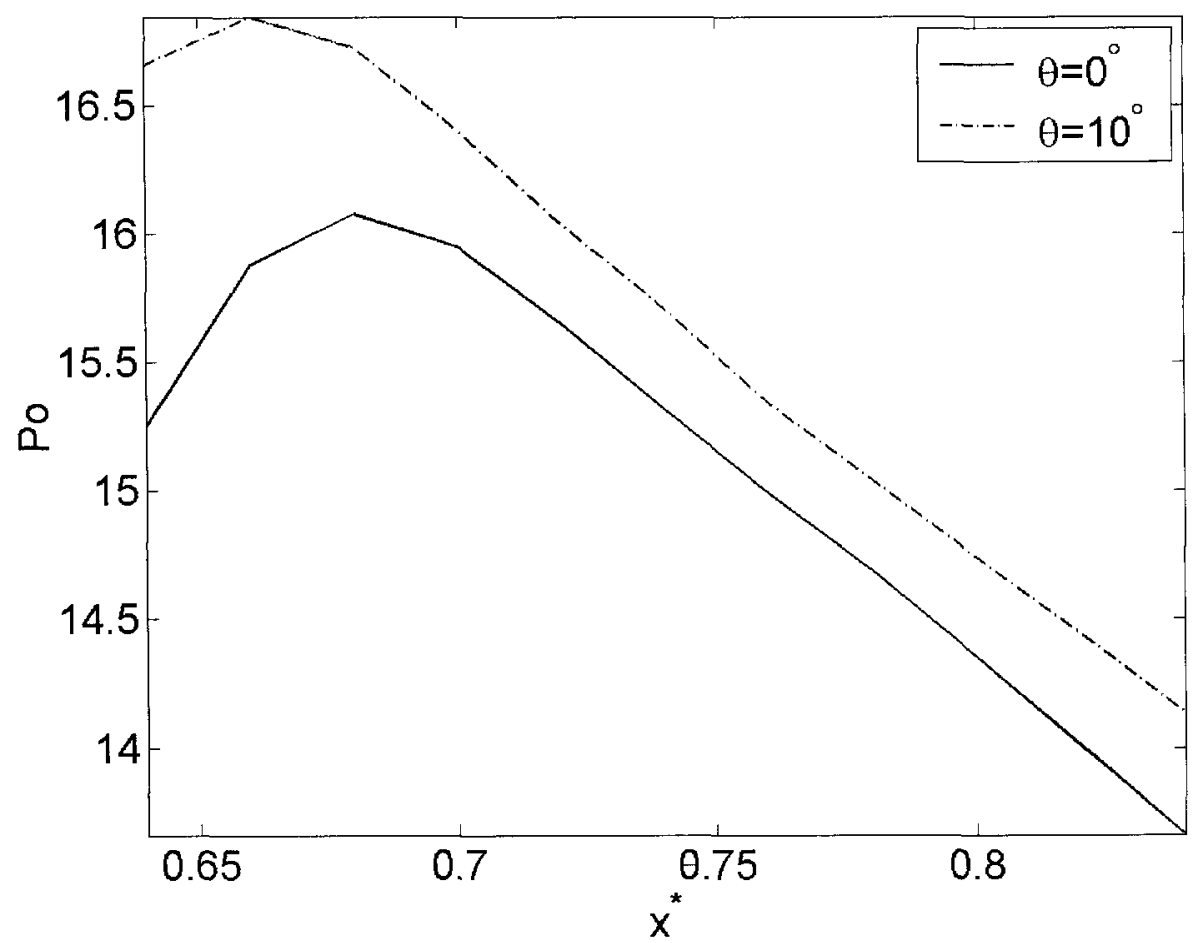

Figure 3.31: Po Variation with Attachment Point, Vapour Counterflow

is partially due to the decrease in the length of the interface as seen in Figure 3.11. The rise in Poiseuille number at the lower attachment points is caused by the rise in the area to perimeter ratio as seen in Figure 3.10. The greater area to perimeter ratio for $\theta=10^{\circ}$ is thought to be the reason why its corresponding Poiseuille number is greater than with $\theta=0^{\circ}$. 
The variation in capillary limit with attachment point for $\theta=0^{\circ}$ and $\theta=10^{\circ}$ is seen in Figure 3.32. One can see a more clearly defined peak in Figure 3.32 compared to Figure 3.30. The heat transfer is also lower for the vapour counterflow condition.

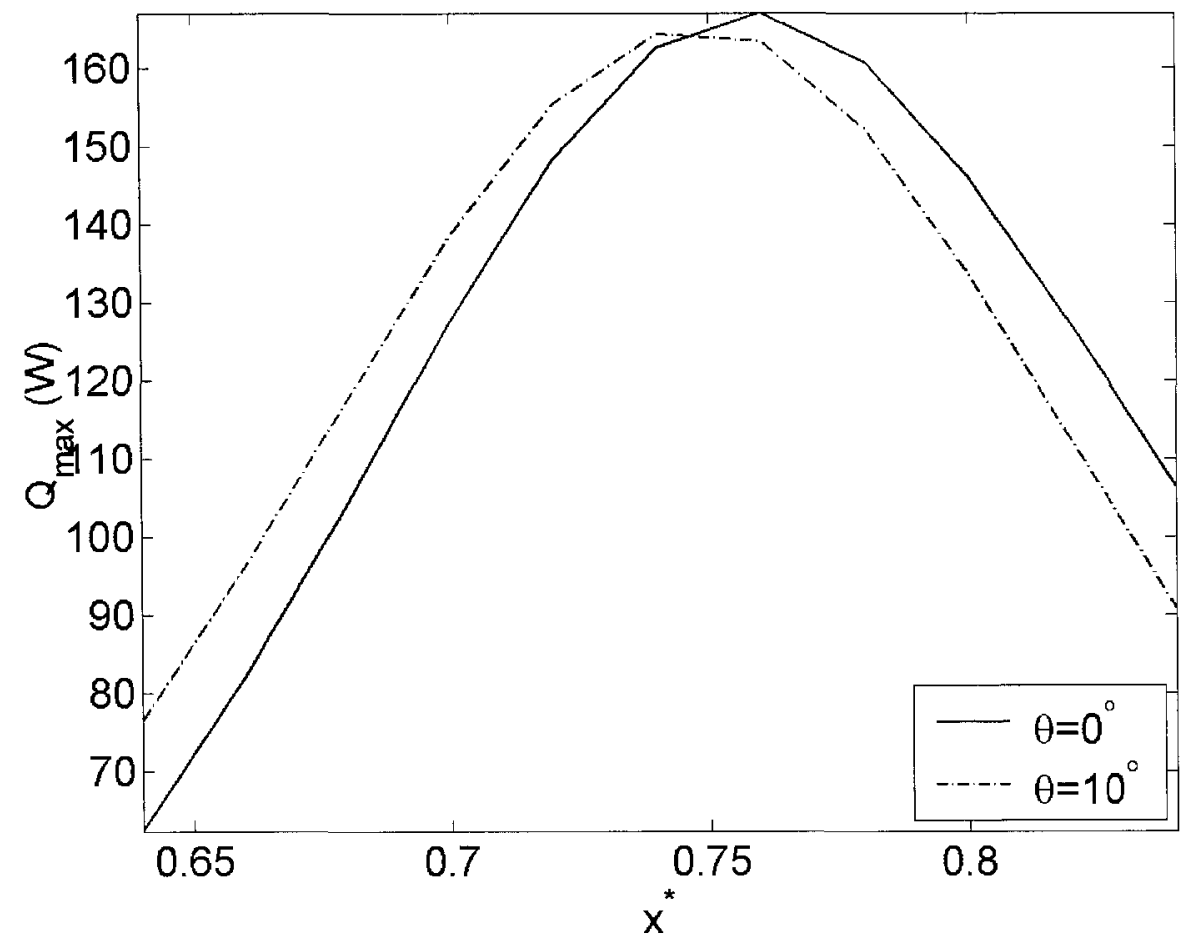

Figure 3.32: Capillary Limit Variation with Attachment Point, Vapour Counterflow 


\subsubsection{Comparison with Experiment}

From laboratory testing of the AGHP, dryout was reached at $\psi=4^{\circ}$ inclination and $5 \mathrm{~W}$ power input. The highest inclination without dryout was with $\psi=3^{\circ}$. Dryout was reached at $200 \mathrm{~W}$ with $\psi=0^{\circ}$. The highest heat input without dryout was 190 W.

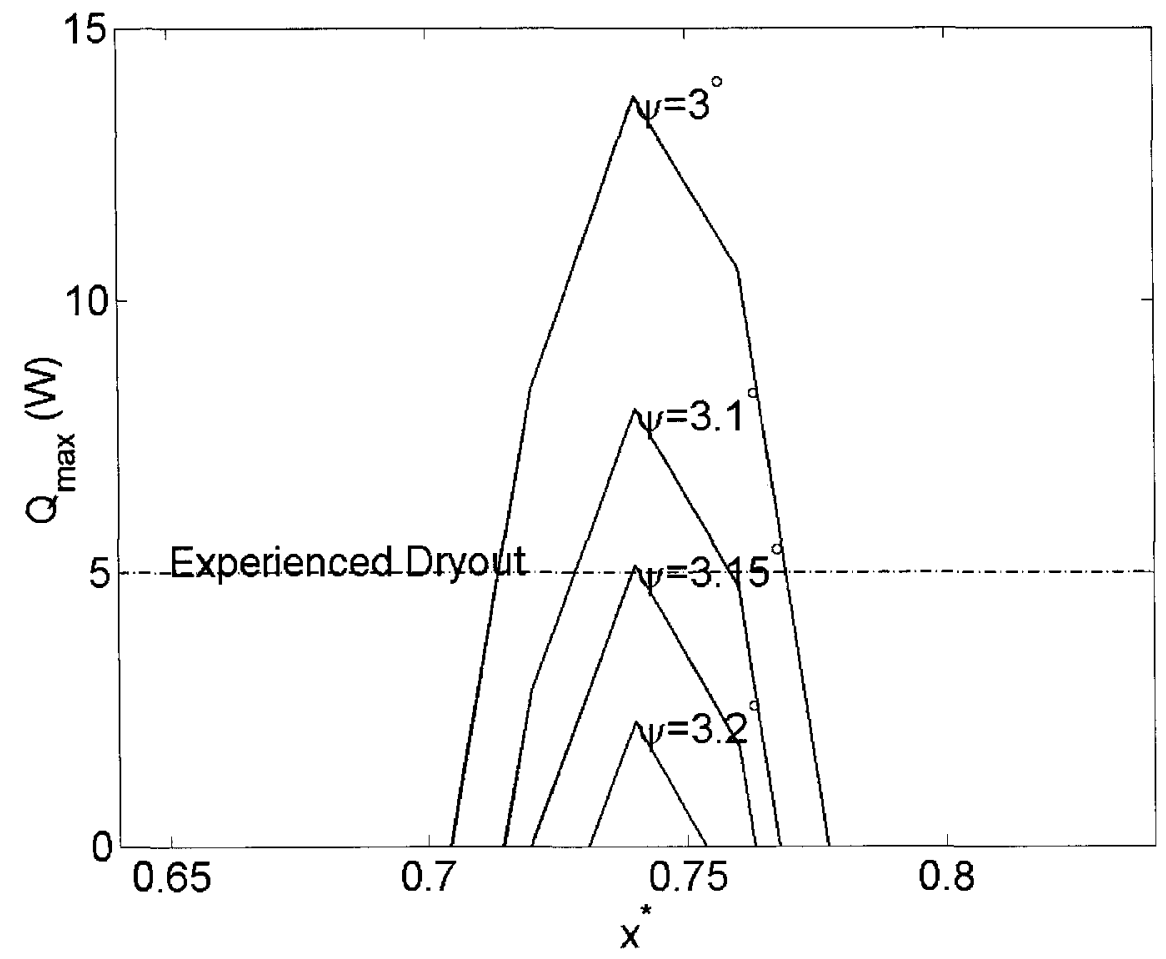

Figure 3.33: Capillary Limit Variation with Inclination Angle, No Slip

The reaction of the heat pipe subject to a body force is modelled in the domain of the same magnitude causing dryout during experimentation to provide a basis for comparison. The Poiseuille number stays the same as with $\psi=0^{\circ}$. Modelling is done with $\theta=0^{\circ}$ because it has proven to deliver the highest heat transfer in the previous analysis. Variation in capillary limit with attachment point and inclination 
angle is shown in Figure 3.33 and Figure 3.34 for the no slip and vapour counterflow, respectively.

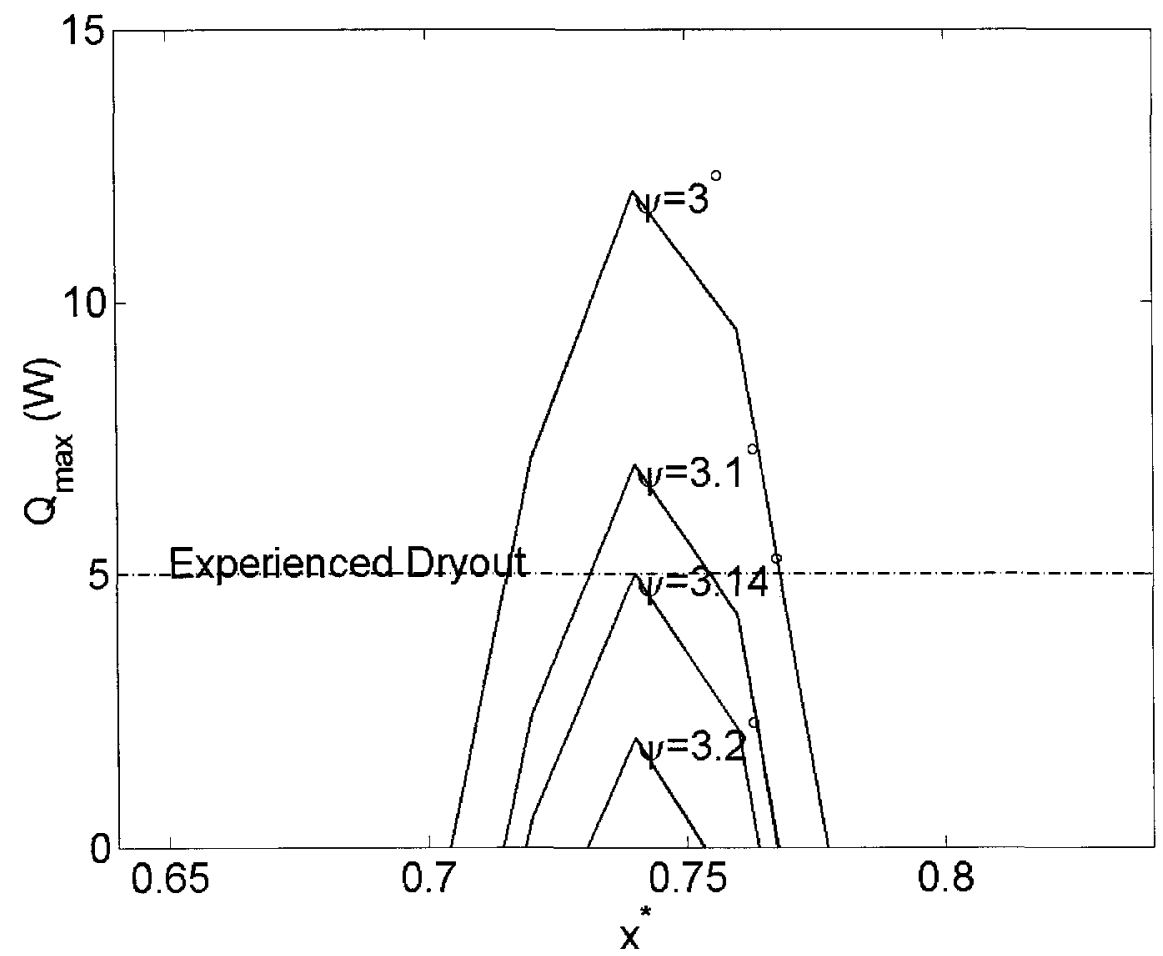

Figure 3.34: Capillary Limit Variation with Inclination Angle, Vapour Counterflow

Dryout with inclination and $5 \mathrm{~W}$ input is predicted to occur between $\psi=3^{\circ}-$ $4^{\circ}$ inclination in both Figure 3.33 and Figure 3.34. Specifically, the heat pipe is predicted to sustain $\psi=3^{\circ}$ at $x^{*}=0.7153$ to $x^{*}=0.7682$ or a maximum of $\psi=3.15^{\circ}$ at $x^{*}=0.74$ with no slip and a $5 \mathrm{~W}$ heat input. The heat pipe is predicted to sustain $\psi=3^{\circ}$ at $x^{*}=0.7153$ to $x^{*}=0.7681$ or a maximum of $\psi=3.14^{\circ}$ at $x^{*}=0.74$ with vapour counterflow and a $5 \mathrm{~W}$ heat input.

Dryout with $\psi=0^{\circ}$ is predicted to occur at $186 \mathrm{~W}$ for the no-slip condition and $167 \mathrm{~W}$ for the vapour counterflow condition. Given limited experimental resolution, 
it was not possible to obtain the exact dryout point. Assuming the actual dryout is at the midpoint of the power settings before and after dryout, the percent difference is $4.62 \%$ and $14.36 \%$ for the no-slip and vapour counterflow case, respectively.

Maximum heat transfer occurred at $x^{*}=0.74$ (groove neck) under inclination as opposed to $x^{*}=0.76$ without inclination. The no-slip and vapour counterflow cases converge with increasing inclination. These occurrences are due to the dominance and independence with respect to boundary conditions of the capillary pressure term.

Capillary limit prediction was more accurate for no slip compared with vapour counterflow. The many simplifying assumptions made led to the overall differences. Given that both models underestimate the capillary heat transfer limit in both horizontal and inclined positions, they can be used as conservative heat pipe design tools. 


\section{Chapter 4}

\section{Conclusions and Recommendations}

\subsection{Conclusions}

\subsubsection{Experimental Study}

A heat pipe stand for testing at various orientations was designed and manufactured. An AGHP and an AHP were tested in ambient with variable heat input at constant sink temperature and variable sink temperature with constant heat input. Capillary heat transfer limits with $\psi=0^{\circ}$ and adverse tilt were determined. Effective thermal conductivity was estimated for both heat pipes based on evaporator and condenser wall temperatures.

The AGHP had a higher effective thermal conductivity than the AHP. Effective thermal conductivity decreased with increasing heat input for both heat pipes. Effective thermal conductivity was more a function of sink temperature at lower heat inputs and more so for the AGHP compared to the AHP. A sharp drop in effective thermal conductivity followed by near constance occurred with the AHP compared to the nearly linear decline seen with the AGHP. 
The AHP sustained a higher heat input than the AGHP without inclination. Both heat pipes dried out at $4^{\circ}$ with $5 \mathrm{~W}$ heat input. Testing with the heater above and below the evaporator showed no significant difference in performance for the AHP. Testing with an inclination yielding a favourable pressure gradient gave a smaller temperature difference between the evaporator and condenser for the AGHP. Hysteresis was not observed in the AGHP or AHP.

\subsubsection{Numerical Study}

A numerical model to determine the capillary limit of an AGHP was developed. A sample cross-section of the AGHP was measured using a profile projector to create the numerical domain for the circumferential re-entrant grooves. The points measured were fit to a 6 th order polynomial to provide continuity. Poiseuille flow was imposed in the liquid groove and a FEM solution was obtained. No-slip and vapour counterflow boundaries were applied at the liquid-vapour interface. No-slip was applied at the solid-liquid interface. The shear value for the vapour counterflow condition was obtained by balancing the mass flow rate between the liquid and vapour and equating the liquid and vapour shear stress at the interface. The effect of a gravitational field on a curved heat pipe was dealt with by analyzing heat pipe segments individually. A parametric analysis with variation of attachment point and contact angle was performed.

It was found that heat transfer is highly dependent on both the attachment point and contact angle at the liquid-vapour interface. Maximum heat transfer was found near the neck on the side closest to the heat pipe centre axis. Additional heat transfer can be obtained by either increasing the cross-sectional area or increasing the maximum capillary pressure. This means that the location of the attachment 
point for maximum heat transfer may not necessarily be at the groove neck even though that is the location that allows for maximum capillary pressure.

Dryout with inclination occurred between $3^{\circ}-4^{\circ}$ and was predicted to occur at $3.15^{\circ}$ and $3.14^{\circ}$ for the no-slip and vapour counterflow models, respectively. The capillary pressure term was found to be more important under inclination compared to horizontal analysis. No-slip and vapour counterflow cases converge with increasing inclination angle due to the capillary pressure's invariance with respect to boundary conditions.

For dryout testing with $\psi=0^{\circ}$, a percent difference of $4.62 \%$ and $14.36 \%$ was found for the no-slip and vapour counterflow models, respectively. The experimental dryout value assumed for percent difference calculation was the average between when dryout was observed and the power input prior to dryout.

Agreement was found between experimental and numerical results. The code developed can be used to conservatively predict the performance of heat pipes with complicated axial groove shapes.

\subsection{Recommendations}

\subsubsection{Experimental Recommendations}

More accurate test results can be obtained with the following changes to the experimental setup:

- Floor stand solely dedicated to heat pipe testing to accept multiple heat pipes of different sizes. This could allow for the two heat pipes to be outfitted with 
thermocouples only once if intermittent testing of the heat pipes is desired. This would reduce wear on the heat pipes and thermocouples.

- Clinometer with greater resolution could be manufactured and integrated with the heat pipe stand for more accurate comparison with the devised numerical model.

- Bearing pivot for the heat pipe stand as opposed to a leg point could provide a smoother transition in variable inclination testing.

- Geared hand crank to change the inclination angle smoothly.

- Heat exchanger supply and return lines made smaller and insulated to decrease the temperature change between the heat sink and exchanger.

- Accounting for enough clearance to place the heat exchanger either above or below the condenser would allow for more testing scenarios.

- Attaching solid u-shaped extensions to the heat exchanger inlet and outlet to eliminate kinks in the coolant lines and create less overall strain on the chiller pump and a more consistent flow rate.

- One heater powerful enough to dryout both heat pipes tested should be used for all tests for more valid comparison. A replica could be made for outfitting both heat pipes at once.

The following tests could be performed to better characterize the heat pipes:

- Dryout testing with maximum favourable pressure gradient could be performed to provide a best case scenario. This would require extra support for the heater that was designed to be supported only in testing with little to no inclination. 
- Horizontal dryout testing of the AHP with the heater below the evaporator could provide a better comparison between the two configurations.

Accounting for the heat balance is critical in determining the effectiveness of the insulation. The following steps can be taken to obtain an accurate heat balance:

- Placing the thermocouples inside the heat exchanger flow would give a more accurate measure of the heat output by the condenser.

- More accurate thermocouples or thermistors.

- Lowering the chiller flow rate would lower the uncertainty in calculating the heat output.

Automation and remote testing could allow for testing during extended periods of time without straining human resources. The following devices can be used to aid in the automation process:

- Integrated power supply unit for easier and faster test point setting during power cycling.

- Automatic heater shutoff switch if the chiller fails or temperature goes beyond acceptable would eliminate undesirable pipe burst.

- Data acquisition software that can also control the hardware such as LabVIEW could allow for standardization and a more elaborate test plan.

\subsubsection{Numerical Recommendations}

Analyzing an AGHP in depth would require three-dimensional Navier-Stokes, energy, and mass transfer equations. Given that a simple yet reasonably accurate model is 
sought, the following assumptions can be added without resorting to the excess of a full solution:

- The analysis of the vapour groove in a similar fashion as the liquid groove to give a more accurate value for the interfacial shear. The vapour flow Poiseuille number could be determined and the validity of using the theoretical value of 16 for a circular section could be tested.

- The introduction of a mass transfer model could lead to prediction of the meniscus shape for a given heat transfer and eliminate the need for contact angle parameterization.

- Creating separate models for the evaporator and condenser would be more realistic to model the entire heat pipe.

Flexibility and user-friendliness can be increased by implementing the following:

- Expanding the existing program to include a GUI.

- Allowing for comparison of various working fluids would make the program a more versatile design aid.

- A generic geometry input for both the liquid groove coordinates and outer dimensions would allow for rapid modelling of various heat pipe configurations.

Relatively little has been published in terms of AHP modelling. Development of an AHP numerical model can be advanced by:

- Modelling Poiseuille flow in the liquid and vapour grooves.

- Creating a capillary limit numerical model. 


\section{References}

[1] Grover, G.M., Cotter, T.P., Erickson G.F., "Structures of Very High Thermal Conductance," Journal of Applied Physics, Vol. 35, No. 6, pp. 1990-1991, 1964.

[2] Richter, R., Gottschlich J. "Thermodynamic Aspects of Heat Pipe Operation," Journal of Thermophysics and Heat Transfer, Vol. 8, No. 2, April-June, pp. 334-340, 1994.

[3] El-Nasr, A.A., El-Hagger, S.M., "Effective Thermal Conductivity of Heat Pipes," International Journal of Heat and Mass Transfer, Vol. 32, No. 1-2, pp. 97-101, 1993.

[4] Jiao, A.J., Riegler, R., "Thin Film Evaporation Effect on Heat Pipe Transport Capability in a Grooved Heat Pipe," Microfluidics and Nanofluidics, Vol. 1, No. 3, pp. 227-233, 2005.

[5] Schlitt, K., Kirkpatrick, J., "Parametric Performance of Extruded Axial Grooved Heat Pipes from 100 to 300K," AIAA-74-724, Thermophysics and Heat Transfer Conference, Boston, Mass., July 15-17, 1974.

[6] Sutara, S.P., Skalak, R., "The History of Poiseille's Law," Annual Review of Fluid Mechanics, Vol. 25, pp. 1-19, 1993.

[7] White, F.M., "Viscous Fluid Flow," McGraw Hill, New York, 1991.

[8] Yovanovich, M.M., Muzychka, Y.S., "Solutions of Poisson Equation Within Singly and Doubly Connected Prismatic Domains," National Heat Transfer Conference, Baltimore, MD, AIAA-1997-3880, 1997.

[9] Thomas, S.K., Damle, V., "Analysis of Fluid Flow in Axial Re-entrant Grooves with Application to Heat Pipes," AIAA-2004-2177 37th AIAA Thermophysics Conference, Portland, Oregon, June 28-July 1, 2004.

[10] Peterson, G., Ma, H., "Analysis of Countercurrent Liquid-Vapor Interactions and the Effect on the Friction Factor," Experimental Thermal and Fluid Science, Vol. 12, No. 1, pp. 13-24, 1996. 
[11] Thomas, S.K., Lykins, R.C., "Fully Developed Laminar Flow in Trapezoidal Grooves with Shear Stress at the Liquid-Vapor Interface," International Journal of Heat and Mass Transfer, Vol. 44 No. 18, pp. 3397-3412, 2001.

[12] Thomas, S.K., Lykins, R.C., "Fully-Developed Laminar Flow in Sinusoidal Grooves," Journal of Fluids Engineering, Vol. 123 No. 3, pp. 656-661, 2001.

[13] Brandt, C., Stephen, P., "Theoretical Investigation of Advanced Capillary Structures in Grooved Heat Pipe Evaporators for Space Applications," 7th European Symposium on Space Environmental Control Systems, SAE 2000-01-2319, Toulouse, 2000.

[14] Ivanovskii, M.N., Sorokin, V.P., "The Physical Principles of Heat Pipes," Calrendon Press, Oxford, 1982.

[15] Chi, F.M., "Heat Pipe Theory and Practice: A Sourcebook," Hemisphere Publishing Corporation, Washington, 1976.

[16] Klasing, K.S., Thomas, S.K., "Caplim: A Visual Basic Program to Calculate the Capillary Limit of an Axially-Grooved Heat Pipe," Energy Conversion Engineering Conference IECEC-97, pp. 1514-1518, Vol.2, July 27-August 1, 1997.

[17] Goncharov, K., Orlov, A., "Arterial Heat Pipe Applications in Russian Spacecrafts," International Journal of Environmentally Conscious Design and Manufacturing, Vol. 9, No. 4, 2000.

[18] Hanford, A.J., Ewert, M.K., "Advanced Active Thermal Control Systems Architecture Study," NASA Technical Memorandum 104822, October, 1996.

[19] Ochterbeck, J.M., Petersont, G.P., "Depriming and Rewetting of Arterial Heat Pipes: Comparison with Share-II Flight Experiment," Journal of Thermophysics and Heat Transfer, Vol. 9, No. 1, January-March, pp. 101-108, 1995.

[20] Golovin, O., Goncharov, A., "Arterial Heat Pipe (AHP) and Axial Grooved Heat Pipe (AGHP) Instruction Manual TC-7055-0," Moscow, 2004.

[21] "Lytron Chiller Instruction Manual," Manual \# 820-0109, October 2003.

[22] Touloukian, Y.S., "Thermophysical Properties of Matter: Specific Heat," Plenum, New York, 1970.

[23] Kline, S.J., "The Purposes of Uncertainty Analysis," Journal of Fluids Engineering, Vol. 107, pp. 153-160, 1985.

[24] Hoffmann, K.A., "Computational Fluid Dynamics," Engineering Education System, Witchita, Kansas, 2000. 
[25] Korn, G.A., Korn, T.M., "Mathematical Handbook for Scientists and Engineers," Dover, New York, 2000. 